\title{
LINEARIZED FIELDS FOR CAUSAL VARIATIONAL PRINCIPLES: EXISTENCE THEORY AND CAUSAL STRUCTURE
}

\author{
CLAUDIO DAPPIAGGI AND FELIX FINSTER
}

NOVEMBER 2018 / NOVEMBER 2019

\begin{abstract}
The existence theory for solutions of the linearized field equations for causal variational principles is developed. We begin by studying the Cauchy problem locally in lens-shaped regions, defined as subsets of space-time which admit foliations by surface layers satisfying hyperbolicity conditions. We prove existence of weak solutions and show uniqueness up to vectors in the orthogonal complement of the jets used for testing. The connection between weak and strong solutions is analyzed. Global solutions are constructed by exhausting space-time by lens-shaped regions. We construct advanced and retarded Green's operators and study their properties.
\end{abstract}

\section{Contents}

1. Introduction

2. Preliminaries

2.1. Causal Variational Principles in the Non-Compact Setting

2.2. The Euler-Lagrange Equations and Jet Spaces

2.3. The Linearized Field Equations

2.4. Surface Layer Integrals

2.5. Positive Functionals Arising from Second Variations

3. Hyperbolic Subsets of Space-Time

3.1. Local Foliations by Surface Layers

3.2. Energy Estimates Using the Surface Layer Inner Product

3.3. Alternative Energy Estimates Using Second Variations

3.4. Lens-Shaped Regions and Time Orientation

3.5. The Cauchy Problem and Uniqueness of Strong Solutions

3.6. Weak Solutions of the Cauchy Problem

3.7. Existence of Weak Solutions

3.8. Are Weak Solutions Unique?

3.9. Weak Solutions in the Future and Past

3.10. Restricting and Extending Weak Solutions

3.11. Estimates of the Initial Data

4. Causal Structure and Global Hyperbolicity

4.1. Causal Cones and Transitive Causal Relations

4.2. Definition of Global Retarded Weak Solutions

4.3. Constructing Unique Global Weak Retarded Solutions

4.4. Finite Propagation Speed

4.5. Globally Hyperbolic Space-Times

4.6. Global Foliations by Cauchy Surface Layers

5. Causal Green's Operators and their Properties 
5.1. Past and Spatially Compact Inhomogeneities 45

5.2. Causal Green's Operators 46

5.3. The Causal Fundamental Solution and its Properties 47

5.4. Connection to the Symplectic Form

6. Discussion and Outlook 51

References 53

\section{INTRODUCTION}

This paper is concerned with the initial value problem in the theory of causal fermion systems (for a general introduction to causal fermion systems and the physical context see the textbook [10] or the survey article [18]). The basic object in this theory is the universal measure, being a measure on a set of linear operators on a Hilbert space. The physical equations are formulated via a variational principle for this measure, the causal action principle. Accordingly, the initial value problem consists in finding a minimizing measure subject to the constraints imposed by the initial data. Since the universal measure describes space-time as well as all structures therein, this initial value problem can be understood in analogy to general relativity: it involves constructing the space-time geometry and the matter fields in one step. Due to the nonlinearity of the interaction as described by the causal action principle, this problem is very difficult. The only results in this direction are the existence and uniqueness theorems in [16] which, however, seem too abstract for getting a direct connection or seeing the analogy to the initial value problem for hyperbolic partial differential equations (PDEs).

Here we are more modest and merely consider the initial value problem for the linearized field equations of a causal fermion system. In the analogous setting of general relativity, this linearization corresponds to studying the initial value problem for linearized fields (like for example the Maxwell field, linearized gravity or the Dirac field) in a given space-time geometry. In the setting of causal fermion systems, such a linearized field is described by a so-called jet $\mathfrak{v}=(b, v)$, which consist of a scalar function $b$ and a vector field $v$ (for details see (2.7) in Section 2.2 below). The jet formalism was introduced in [20] for causal variational principles, which are a mathematical generalization of the causal action principle. For convenience and for the sake of larger generality, here we also work in the setting of causal variational principles (the necessary preliminaries will be given in Section 2). The main objective of the present paper is to show that energy methods for hyperbolic PDEs can be adapted to the setting of causal variational principles such as to obtain existence and uniqueness results for solutions to the initial value problem for linearized fields. Moreover, we prove that the linearized fields propagate with finite speed. We also analyze the resulting causal structure.

We note that our methods and results for linearized fields are also a good starting point for tackling the nonlinear problem. Namely, the existence theory for the linearized field equations opens the door for also adapting nonlinear methods from PDEs (like fixed-point methods for nonlinear symmetric hyperbolic systems [36] or methods developed for the Einstein equations [6, 34, 35]). Moreover, our results for linearized fields can be applied directly to the general perturbation expansion for the universal measure as developed in [14]. Indeed, this perturbative description, which resembles 
the Feynman diagram expansion of quantum field theory, relies heavily on Green's operators for the linearized fields. In the present paper, we shall prove under general assumptions that these Green's operators exist and have all the properties needed for the perturbative treatment.

The analogy between linear hyperbolic PDEs and the linearized field equations for causal variational principles deserves a few general words. The linearized field equations take the form

$$
\Delta \mathfrak{v}=\mathfrak{w}
$$

where $\mathfrak{w}$ is a given inhomogeneity, and the operator $\Delta$ is defined by

$$
\Delta \mathfrak{v}(x)=\nabla\left(\int_{M}\left(\nabla_{1, \mathfrak{v}}+\nabla_{2, \mathfrak{v}}\right) \mathcal{L}(x, y) d \rho(y)-\nabla_{\mathfrak{v}} \mathfrak{s}\right)
$$

where $\mathcal{L}$ is the Lagrangian of the causal variational principle, space-time $M$ is defined as the support of the universal measure $\rho$ (for details see Section 2.1), $\mathfrak{s}$ is a positive parameter, and the jet derivative $\nabla$ is a combination of multiplication and directional derivative (for details see Sections 2.2 and 2.3). One should keep in mind that these equations are not differential equations, but instead they are nonlocal equations involving space-time integrals of specific integral kernels. The reason why, despite these major structural differences, methods of hyperbolic PDEs are applicable is that there are positive energies which can be controlled in time by suitable energy estimates. Once these positive energies have been identified, we can closely follow the procedure for linear symmetric hyperbolic systems as introduced in [26] (see also the textbooks [8, 31, 36, 22] or similarly in globally hyperbolic space-times [34, 35, 1]).

We now explain our constructions and results more concretely. Recall that, in order to set up the initial value problem for a linear hyperbolic PDE in a Lorentzian spacetime $(\mathscr{M}, g)$, one chooses a smooth family $\left(\mathcal{N}_{t}\right)_{t \in\left[t_{0}, t_{\max }\right]}$ of space-like hypersurfaces, which can be thought of as the surfaces of constant time $t$ of an observer. Given initial data on $\mathcal{N}_{t_{0}}$, one seeks for a solution of the linear hyperbolic equation in the spacetime region $L:=\cup_{t \in\left[t_{0}, t_{\text {max }}\right]} \mathcal{N}_{t} \subset \mathcal{M}$ (see for example the textbooks [31, Section 5.3], [36, Section 16], [34, Section 8.3] or [22, Chapter 11]). The family $\left(\mathcal{N}_{t}\right)_{t \in\left[t_{0}, t_{\max }\right]}$ is sometimes referred to as a foliation of the lens-shaped region $L$. In the setting of causal variational principles, the situation is more intricate for two reasons: First, space-time could be discrete, in which case an above foliation does not exist. Second, it is not clear what an integral over a hypersurface should be, making it impossible to work with function spaces at fixed times. The method to overcome these difficulties is to replace hypersurfaces by so-called surface layers, as we now explain. In the above example of a Lorentzian space-time, we can introduce functions $\eta_{t}$ as the characteristic functions of the past of $\mathcal{N}_{t}$. Then their derivative $\partial_{t} \eta_{t}$ is a $\delta$-distribution supported on the surface $\mathcal{N}_{t}$. Likewise, integrals over $\mathcal{N}_{t}$ can be written as space-time integrals involving the distribution $\partial_{t} \eta_{t}$. In the setting of causal variational principles, on the other hand, space-time $M$ is by definition the support of the universal measure $\rho$. We choose a family of non-negative functions $\left(\eta_{t}\right)_{t \in\left[t_{0}, t_{\max }\right]}$ defined in a space-time region $U \subset M$. These functions should be equal to one in the past and equal to zero in the future, interpolating smoothly between these two values in a neighborhood of a hypersurface. Moreover, we assume that the "time" derivative $\theta_{t}:=\partial_{t} \eta_{t}$ exists and is non-negative. Then the function $\theta_{t}$ is supported in a neighborhood of the hypersurface. Using a notion first introduced in [19], we refer to the support of $\theta_{t}$ as a surface layer. 
The integral

$$
\int_{U} \theta_{t}(x) \cdots d \rho(x)
$$

should be thought of as the generalization of an integral over a hypersurface to the setting of causal variational principles. The integral is not localized on a hypersurface, but instead it is "smeared out" in a small "time strip" around the hypersurface. This picture is made precise by the notion of a local foliation by surface layers (see Definition 3.1).

Working with surface layers is well-suited to our problem also because the above "time strips" reflect the nonlocality of the linearized field equations. Moreover, integral estimates in "time strips" harmonize with the conservation laws for surface layer integrals as found in [20, 21] (for basics see Section 2.4). In order for these structures to fit together even better, we here write the surface layer integrals as

$$
\int_{U} \eta_{t}(x) d \rho(x) \int_{U}\left(1-\eta_{t}(y)\right) d \rho(y)(\cdots) \mathcal{L}(x, y),
$$

where $(\cdots)$ is a differential operator involving the jets.

Working in the above setup with suitable energies and imposing corresponding hyperbolicity conditions, we obtain energy estimates which in turn give rise to the desired existence and uniqueness results. We consider two alternative energies. The first energy is the surface layer inner product $(., .)^{t}$ introduced in [21] (see Section 3.2). This choice is motivated from the physical applications in which $(., .)^{t}$ gives the scalar product of quantum theory (see [13, 17]). The second energy, denoted by $\langle., .\rangle_{\left[t_{0}, t\right]}$, arises in the study of second variations [12] (see Section 3.3). While it does not have an immediate physical interpretation, it has the advantage that it is positive as a consequence of the mathematical structure of causal variational principles. In both cases, the hyperbolicity condition is stated as a positivity property of the respective energy (see Definitions 3.3 and 3.77). A lens-shaped region $L$ is defined as a subset of space-time which admits a local foliation by surface layers which satisfies one of the alternative hyperbolicity conditions (see Definition 3.11). In a lens-shaped region, we set up the Cauchy problem and prove uniqueness (see Proposition 3.12). Moreover, we introduce the notion of a weak solution, defined by the equation

$$
\langle\Delta \mathfrak{u}, \mathfrak{v}\rangle_{L^{2}(L)}=\langle\mathfrak{u}, \mathfrak{w}\rangle_{L^{2}(L)}
$$

which must hold for all test jets $\mathfrak{u}$ in a suitable jet space (for details see Section 3.6). We prove existence of weak solutions (Theorems 3.15 and 3.18 ).

Our uniqueness statement for weak solutions requires an explanation. As mentioned above, we want to allow for the possibility that space-time is discrete or has some other, yet unknown microstructure. In such situations, the hyperbolicity conditions mentioned above are typically known to be satisfied only on the macroscopic scale, i.e. for jets which are almost constant on the microscopic scale and thus do not "see" the unknown microstructure. This concept can be made precise in the weak formulation by testing only with jets which are almost constant on the microscopic scale. In order to allow for such situations, we do not specify the jet space used for testing. In particular, we do not assume that the test jets are dense in $L^{2}(L)$. As a consequence, the weak equation determines the solution only up to vectors in the orthogonal complement of the test jets. Except for this obvious freedom, weak solutions are unique (see Proposition 3.16). 
With the methods and results explained so far, one can solve the Cauchy problem "locally" in a lens-shaped region. In order to construct global solutions, one must extend local solutions and prove that the resulting globally defined jets satisfy the linearized field equations. Our method for extending a solution $\mathfrak{v}$ from $L$ to $\tilde{L} \supset L$ is to enlarge the test space to jets supported in the bigger space-time region $\tilde{L}$. In view of the above-mentioned freedom in modifying weak solutions, the extension will coincide with $\mathfrak{v}$ in $L$ only up to a jet in the orthogonal complement of the test jets in $L$. This is a delicate point which we handle using the concept of shielding (see Definitions 3.21 and 4.8 as well as the shielding condition (4.15)). We thus succeed in proving existence of global weak solutions under general assumptions (Theorem 4.9 and Corollary 5.2).

In view of the fact that the solution of the Cauchy problem for zero initial data vanishes identically in the whole lens-shaped region, lens-shaped regions tell us about the speed of propagation of linearized solutions. Using this information systematically, we construct future cones (see Definition 4.3). The relation "lies in the future of" induced by the open future cones is transitive (Theorem 4.5). Moreover, the cone structure is compatible with the causal propagation speed (as is made precise in Theorem 4.16). Combining all the assumptions needed for our constructions leads to the notion of globally hyperbolic space-times (see Definition 4.20).

We finally construct advanced and retarded Green's operators $S^{\wedge}$ and $S^{\vee}$ (see (5.2) and Corollary [5.2). The difference of these Green's operators $G$ maps to the homogeneous linearized solutions (see (5.5)). We show that the operators $\Delta$ and $G$ have useful properties which are summarized in the exact sequence

$$
0 \rightarrow \mathfrak{J}_{0}^{\text {test }} \stackrel{\Delta}{\longrightarrow} \mathfrak{J}_{0}^{*} \stackrel{G}{\longrightarrow} \mathfrak{J}_{\mathrm{sc}} \stackrel{\Delta}{\longrightarrow} \mathfrak{J}_{\mathrm{sc}}^{*} \rightarrow 0
$$

where $\mathfrak{J}_{0}^{\text {test }}$ and $\mathfrak{J}_{0}^{*}$ are spaces of compactly supported jets, whereas $\mathfrak{J}_{\mathrm{sc}}$ and $\mathfrak{J}_{\mathrm{sc}}^{*}$ have spatially compact support (see Theorem 5.7).

The paper is organized as follows. In Section 2 we provide the necessary preliminaries. Section 3 is devoted to the Cauchy problem in a lens-shaped region. In Section 4 the causal structure of linearized fields is worked out, and it is analyzed how and under which assumptions one can construct global solutions. In Section 5 causal Green's operators are introduced, and their properties are analyzed. in Section 6 we conclude with a discussion and an outlook on open problems.

\section{Preliminaries}

2.1. Causal Variational Principles in the Non-Compact Setting. We consider causal variational principles in the non-compact setting as introduced in [20, Section 2]. Thus we let $\mathcal{F}$ be a (possibly non-compact) smooth manifold of dimension $m \geq 1$ and $\rho$ a (positive) Borel measure on $\mathcal{F}$ (the universal measure). Moreover, we are given a non-negative function $\mathcal{L}: \mathcal{F} \times \mathcal{F} \rightarrow \mathbb{R}_{0}^{+}$(the Lagrangian) with the following properties:

(i) $\mathcal{L}$ is symmetric: $\mathcal{L}(x, y)=\mathcal{L}(y, x)$ for all $x, y \in \mathcal{F}$.

(ii) $\mathcal{L}$ is lower semi-continuous, i.e. for all sequences $x_{n} \rightarrow x$ and $y_{n^{\prime}} \rightarrow y$,

$$
\mathcal{L}(x, y) \leq \liminf _{n, n^{\prime} \rightarrow \infty} \mathcal{L}\left(x_{n}, y_{n^{\prime}}\right) .
$$

The causal variational principle is to minimize the action

$$
\mathcal{S}(\rho)=\int_{\mathcal{F}} d \rho(x) \int_{\mathcal{F}} d \rho(y) \mathcal{L}(x, y)
$$


under variations of the measure $\rho$, keeping the total volume $\rho(\mathcal{F})$ fixed (volume constraint). The notion causal in "causal variational principles" refers to the fact that the Lagrangian induces on $M$ a causal structure given by

$$
x, y \in M \text { are }\left\{\begin{array}{c}
\text { timelike } \\
\text { spacelike }
\end{array}\right\} \text { separated if }\left\{\begin{array}{l}
\mathcal{L}(x, y)>0 \\
\mathcal{L}(x, y)=0
\end{array}\right\} .
$$

The connection between this notion of causality and the causal structure of linearized fields will be discussed in Section 6. An important example of causal variational principles is the causal action principle for causal fermion systems (for the connection see [20, Section 2]). In this case, the Lagrangian is even continuous. The more general lower semi-continuous setting arises when optimizing the hypothesis needed for getting a mathematically well-posed problem. Moreover, lower semicontinuity arises in the context of causal fermion systems when integrating out degrees of freedom and applying Fatou's lemma (as explained for static causal fermion systems in [24, Section 3.3]).

If the total volume $\rho(\mathcal{F})$ is finite, one minimizes (2.1) over all regular Borel measures with the same total volume. If the total volume $\rho(\mathcal{F})$ is infinite, however, it is not obvious how to implement the volume constraint, making it necessary to proceed as follows. We need the following additional assumptions:

(iii) The measure $\rho$ is locally finite (meaning that any $x \in \mathcal{F}$ has an open neighborhood $U$ with $\rho(U)<\infty)$.

(iv) The function $\mathcal{L}(x,$.$) is \rho$-integrable for all $x \in \mathcal{F}$, giving a lower semi-continuous and bounded function on $\mathcal{F}$.

Given a regular Borel measure $\rho$ on $\mathcal{F}$, we then vary over all regular Borel measures $\tilde{\rho}$ with

$$
|\tilde{\rho}-\rho|(\mathcal{F})<\infty \quad \text { and } \quad(\tilde{\rho}-\rho)(\mathcal{F})=0
$$

(where |.| denotes the total variation of a measure). These variations of the causal action are well-defined. The existence theory for minimizers is developed in [23].

We point out that, since a manifold is by definition locally compact and separable, $\mathcal{F}$ is a $\sigma$-compact topological space. As a consequence, every closed subset of $\mathcal{F}$ is also $\sigma$-compact; this fact will be used later on.

2.2. The Euler-Lagrange Equations and Jet Spaces. A minimizer of the causal variational principle satisfies the following Euler-Lagrange (EL) equations: For a suitable value of the parameter $\mathfrak{s}>0$, the lower semi-continuous function $\ell: \mathcal{F} \rightarrow \mathbb{R}_{0}^{+}$ defined by

$$
\ell(x):=\int_{\mathcal{F}} \mathcal{L}(x, y) d \rho(y)-\mathfrak{s}
$$

is minimal and vanishes on space-time $M:=\operatorname{supp} \rho$,

$$
\left.\ell\right|_{M} \equiv \inf _{\mathcal{F}} \ell=0 \text {. }
$$

The parameter $\mathfrak{s}$ can be understood as the Lagrange parameter corresponding to the volume constraint. For the derivation and further details we refer to [20, Section 2].

The EL equations (2.4) are nonlocal in the sense that they make a statement on the function $\ell$ even for points $x \in \mathcal{F}$ which are far away from space-time $M$. It turns out that for the applications we have in mind, it is preferable to evaluate the EL equations only locally in a neighborhood of $M$. This leads to the weak EL equations introduced in [20, Section 4]. Here we give a slightly less general version of these equations which is sufficient for our purposes. In order to explain how the weak EL equations come 
about, we begin with the simplified situation that the function $\ell$ is smooth. In this case, the minimality of $\ell$ implies that the derivative of $\ell$ vanishes on $M$, i.e.

$$
\left.\ell\right|_{M} \equiv 0 \quad \text { and }\left.\quad D \ell\right|_{M} \equiv 0
$$

(where $D \ell(p): T_{p} \mathcal{F} \rightarrow \mathbb{R}$ is the derivative). In order to combine these two equations in a compact form, it is convenient to consider a pair $\mathfrak{u}:=(a, u)$ consisting of a real-valued function $a$ on $M$ and a vector field $u$ on $T \mathcal{F}$ along $M$, and to denote the combination of multiplication of directional derivative by

$$
\nabla_{\mathfrak{u}} \ell(x):=a(x) \ell(x)+\left(D_{u} \ell\right)(x) .
$$

Then the equations (2.5) imply that $\nabla_{\mathfrak{u}} \ell(x)$ vanishes for all $x \in M$. The pair $\mathfrak{u}=(a, u)$ is referred to as a jet.

In the general lower-continuous setting, one must be careful because the directional derivative $D_{u} \ell$ in (2.6) need not exist. Our method for dealing with this problem is to restrict attention to vector fields for which the directional derivative is well-defined. Moreover, we must specify the regularity assumptions on $a$ and $u$. To begin with, we always assume that $a$ and $u$ are smooth in the sense that they have a smooth extension to the manifold $\mathcal{F}$. Thus the jet $\mathfrak{u}$ should be an element of the jet space

$$
\mathfrak{J}:=\left\{\mathfrak{u}=(a, u) \text { with } a \in C^{\infty}(M, \mathbb{R}) \text { and } u \in \Gamma(M, T \mathcal{F})\right\},
$$

where $C^{\infty}(M, \mathbb{R})$ and $\Gamma(M, T \mathcal{F})$ denote the space of real-valued functions and vector fields on $M$, respectively, which admit a smooth extension to $\mathcal{F}$.

Clearly, the fact that a jet $\mathfrak{u}$ is smooth does not imply that the functions $\ell$ or $\mathcal{L}$ are differentiable in the direction of $\mathfrak{u}$. This must be ensured by additional conditions which are satisfied by suitable subspaces of $\mathfrak{J}$ which we now introduce. First, we let $\Gamma^{\text {diff }}$ be those vector fields for which the directional derivative of the function $\ell$ exists,

$$
\Gamma^{\text {diff }}=\left\{u \in C^{\infty}(M, T \mathcal{F}) \mid D_{u} \ell(x) \text { exists for all } x \in M\right\} .
$$

This gives rise to the jet space

$$
\mathfrak{J}^{\text {diff }}:=C^{\infty}(M, \mathbb{R}) \oplus \Gamma^{\text {diff }} \subset \mathfrak{J} .
$$

For the jets in $\mathfrak{J}^{\text {diff }}$, the combination of multiplication and directional derivative in (2.6) is well-defined. We choose a linear subspace $\mathfrak{J}^{\text {test }} \subset \mathfrak{J}^{\text {diff }}$ with the property that its scalar and vector components are both vector spaces,

$$
\mathfrak{J}^{\text {test }}=C^{\text {test }}(M, \mathbb{R}) \oplus \Gamma^{\text {test }} \subseteq \mathfrak{J}^{\text {diff }},
$$

and the scalar component is nowhere trivial in the sense that

$$
\text { for all } x \in M \text { there is } a \in C^{\text {test }}(M, \mathbb{R}) \text { with } a(x) \neq 0 \text {. }
$$

Then the weak EL equations read (for details cf. [20, (eq. (4.10)])

$$
\left.\nabla_{\mathfrak{u}} \ell\right|_{M}=0 \quad \text { for all } \mathfrak{u} \in \mathfrak{J}^{\text {test }} .
$$

The purpose of introducing $\mathfrak{J}^{\text {test }}$ is that it gives the freedom to restrict attention to the portion of information in the EL equations which is relevant for the application in mind. For example, if one is interested only in the macroscopic dynamics, one can choose $\mathfrak{J}^{\text {test }}$ to be composed of jets pointing in directions where the microscopic fluctuations of $\ell$ are disregarded.

Before going on, we point out that the weak EL equations (2.9) do not hold only for minimizers, but also for critical points of the causal action. With this in mind, all methods and results of this paper (except for the constructions using second variations 
in Sections 2.5 and 3.3 do not apply only to minimizers, but more generally to critical points of the causal variational principle. For brevity, we also refer to a measure with satisfies the weak EL equations (2.9) as a critical measure.

We conclude this section by introducing a few other jet spaces which will be needed later on. It is useful to define the differentiability properties of the jets by corresponding differentiability properties of the Lagrangian. When considering higher derivatives, we always choose charts and work in components. For ease in notation, we usually omit all vector and tensor indices. But one should keep in mind that, from now on, we always work in suitably chosen charts. We first introduce the jet spaces $\mathfrak{J}^{\ell}$, where $\ell \in \mathbb{N} \cup\{\infty\}$ can be thought of as the order of differentiability if the derivatives act simultaneously on both arguments of the Lagrangian:

Definition 2.1. For any $\ell \in \mathbb{N}_{0} \cup\{\infty\}$, the jet space $\mathfrak{J}^{\ell} \subset \mathfrak{J}$ is defined as the vector space of jets with the following properties:

(i) For all $y \in M$ and all $x$ in an open neighborhood of $M$, the directional derivatives

$$
\left(\nabla_{1, \mathfrak{v}_{1}}+\nabla_{2, \mathfrak{v}_{1}}\right) \cdots\left(\nabla_{1, \mathfrak{v}_{p}}+\nabla_{2, \mathfrak{v}_{p}}\right) \mathcal{L}(x, y)
$$

(computed componentwise in charts around $x$ and $y$ ) exist for all $p \in\{1, \ldots, \ell\}$ and all $\mathfrak{v}_{1}, \ldots, \mathfrak{v}_{p} \in \mathfrak{J}^{\ell}$. Here the subscripts 1,2 refer to the derivatives acting on the first and on the second argument of $\mathcal{L}(x, y)$ respectively.

(ii) The functions in (2.10) are $\rho$-integrable in the variable y, giving rise to locally bounded functions in $x$. More precisely, these functions are in the space

$$
L_{\mathrm{loc}}^{\infty}\left(M, L^{1}(M, d \rho(y)) ; d \rho(x)\right) .
$$

(iii) Integrating the expression (2.10) in y over $M$ with respect to the measure $\rho$, the resulting function (defined for all $x$ in an open neighborhood of $M$ ) is continuously differentiable in the direction of every jet $\mathfrak{u} \in \mathfrak{J}^{\text {test }}$.

Here and throughout this paper, we use the following conventions for partial derivatives and jet derivatives:

- Partial and jet derivatives with an index $i \in\{1,2\}$, as for example in (2.10), only act on the respective variable of the function $\mathcal{L}$. This implies, for example, that the derivatives commute,

$$
\nabla_{1, \mathfrak{v}} \nabla_{1, \mathfrak{u}} \mathcal{L}(x, y)=\nabla_{1, \mathfrak{u}} \nabla_{1, \mathfrak{v}} \mathcal{L}(x, y) .
$$

- The partial or jet derivatives which do not carry an index act as partial derivatives on the corresponding argument of the Lagrangian. This implies, for example, that

$$
\nabla_{\mathfrak{u}} \int_{\mathcal{F}} \nabla_{1, \mathfrak{v}} \mathcal{L}(x, y) d \rho(y)=\int_{\mathcal{F}} \nabla_{1, \mathfrak{u}} \nabla_{1, \mathfrak{v}} \mathcal{L}(x, y) d \rho(y) .
$$

We point out that, in contrast to the method and conventions used in [20], jets are never differentiated.

We denote the $\ell$-times continuously differentiable test jets by $\mathfrak{J}^{\text {test }} \cap \mathfrak{J}^{\ell}$. Moreover, compactly supported jets are denoted by a subscript zero, like for example

$$
\mathfrak{J}_{0}^{\text {test }}:=\left\{\mathfrak{u} \in \mathfrak{J}^{\text {test }} \mid \mathfrak{u} \text { has compact support }\right\} \text {. }
$$

In order to make sure that surface layer integrals exist (see Section 2.4 below), one needs differentiability conditions of a somewhat different type (for details see [21, Section 3.5]): 
Definition 2.2. The jet space $\mathfrak{J}^{\text {test }}$ is surface layer regular if $\mathfrak{J}^{\text {test }} \subset \mathfrak{J}^{2}$ and if for all $\mathfrak{u}, \mathfrak{v} \in \mathfrak{J}^{\text {test }}$ and all $p \in\{1,2\}$ the following conditions hold:

(i) The directional derivatives

$$
\nabla_{1, \mathfrak{u}}\left(\nabla_{1, \mathfrak{v}}+\nabla_{2, \mathfrak{v}}\right)^{p-1} \mathcal{L}(x, y)
$$

exist.

(ii) The functions in (2.13) are $\rho$-integrable in the variable y, giving rise to locally bounded functions in $x$. More precisely, these functions are in the space

$$
L_{\mathrm{loc}}^{\infty}\left(L^{1}(M, d \rho(y)), d \rho(x)\right) .
$$

(iii) The u-derivative in (2.13) may be interchanged with the y-integration, i.e.

$$
\int_{M} \nabla_{1, \mathfrak{u}}\left(\nabla_{1, \mathfrak{v}}+\nabla_{2, \mathfrak{v}}\right)^{p-1} \mathcal{L}(x, y) d \rho(y)=\nabla_{\mathfrak{u}} \int_{M}\left(\nabla_{1, \mathfrak{v}}+\nabla_{2, \mathfrak{v}}\right)^{p-1} \mathcal{L}(x, y) d \rho(y) .
$$

The precise regularity assumptions needed for our applications will be specified below whenever we need them.

We finally introduce the space of dual jets $\left(\mathfrak{J}^{\text {test }}\right)^{*}$. To this end, we denote the continuous global one-jets taking values in the cotangent bundle restricted to $M$ by

$$
\mathfrak{J}^{*}:=C^{0}(M, \mathbb{R}) \oplus C^{0}\left(M, T^{*} \mathcal{F}\right) .
$$

We let $\left(\mathfrak{J}^{\text {test }}\right)^{*}$ be the quotient space

$$
\begin{aligned}
\left(\mathfrak{J}^{\text {test }}\right)^{*}:=\mathfrak{J}^{*} /\left\{(g, \varphi) \in \mathfrak{J}^{*} \mid\right. & g(x) a(x)+\langle\varphi(x), u(x)\rangle=0 \\
& \text { for all } \left.\mathfrak{u}=(a, u) \in \mathfrak{J}^{\text {test }} \text { and all } x \in M\right\},
\end{aligned}
$$

where $\langle.,$.$\rangle denotes the dual pairing of T_{x}^{*} \mathcal{F}$ and $T_{x} \mathcal{F}$. Here we take equivalence classes simply because it is convenient to disregard dual jets which are trivial on $\mathfrak{J}^{\text {test }}$.

2.3. The Linearized Field Equations. In simple terms, the homogeneous linearized field equations describe variations of the universal measure which preserve the EL equations. More precisely, we consider variations where we multiply $\rho$ by a nonnegative function and take the push-forward with respect to a mapping from $M$ to $\mathcal{F}$. Thus we consider families of measures $\left(\tilde{\rho}_{\tau}\right)_{\tau \in(-\delta, \delta)}$ of the form

$$
\tilde{\rho}_{\tau}=\left(F_{\tau}\right)_{*}\left(f_{\tau} \rho\right) \text {, }
$$

where $f$ and $F$ are smooth,

$$
f \in C^{\infty}\left((-\delta, \delta) \times M \rightarrow \mathbb{R}^{+}\right) \quad \text { and } \quad F \in C^{\infty}((-\delta, \delta) \times M \rightarrow \mathcal{F}),
$$

and have the properties $f_{0}(x)=1$ and $F_{0}(x)=x$ for all $x \in M$ (here the pushforward measure is defined for a subset $\Omega \subset \mathcal{F}$ by $\left(\left(F_{\tau}\right)_{*} \mu\right)(\Omega)=\mu\left(F_{\tau}^{-1}(\Omega)\right)$; see for example [4, Section 3.6]). If we demand that $\left(\tilde{\rho}_{\tau}\right)_{\tau \in(-\delta, \delta)}$ is a family of minimizers, the EL equations (2.4) hold for all $\tau$, i.e.

$$
\left.\tilde{\ell}_{\tau}\right|_{M_{\tau}} \equiv \inf _{\mathcal{F}} \ell_{\tau}=0 \quad \text { with } \quad \tilde{\ell}_{\tau}(x):=\int_{\mathcal{F}} \mathcal{L}(x, y) d \tilde{\rho}_{\tau}(y)-\mathfrak{s},
$$

where $M_{\tau}$ is the support of the varied measure,

$$
M_{\tau}:=\operatorname{supp} \tilde{\rho}_{\tau}=\overline{F_{\tau}(M)} .
$$


In (2.15) we can express $\tilde{\rho}$ in terms of $\rho$. Moreover, it is convenient to rewrite this equation as an equation on $M$ and to multiply by $f_{\tau}(x)$. We thus obtain the equivalent equation

$$
\left.\ell_{\tau}\right|_{M} \equiv \inf _{\mathcal{F}} \ell_{\tau}=0
$$

with

$$
\ell_{\tau}(x):=\int_{\mathcal{F}} f_{\tau}(x) \mathcal{L}\left(F_{\tau}(x), F_{\tau}(y)\right) f_{\tau}(y) d \tilde{\rho}_{\tau}(y)-f_{\tau}(x) \mathfrak{s}
$$

In analogy to (2.9) we write the corresponding weak EL equations as

$$
\left.\nabla_{\mathfrak{u}} \ell_{\tau}\right|_{M}=0 \quad \text { for all } \mathfrak{u} \in \mathfrak{J}^{\text {test }}
$$

(for details on why the jet space does not depend on $\tau$ we refer to [14, Section 4.1]). Since this equation holds by assumption for all $\tau$, we can differentiate it with respect to $\tau$. Denoting the infinitesimal generator of the variation by $\mathfrak{v}$, i.e.

$$
\mathfrak{v}(x):=\left.\frac{d}{d \tau}\left(f_{\tau}(x), F_{\tau}(x)\right)\right|_{\tau=0},
$$

we obtain the linearized field equations

$$
0=\langle\mathfrak{u}, \Delta \mathfrak{v}\rangle(x):=\nabla_{\mathfrak{u}}\left(\int_{M}\left(\nabla_{1, \mathfrak{v}}+\nabla_{2, \mathfrak{v}}\right) \mathcal{L}(x, y) d \rho(y)-\nabla_{\mathfrak{v}} \mathfrak{s}\right),
$$

which are to be satisfied for all $\mathfrak{u} \in \mathfrak{J}^{\text {test }}$ and all $x \in M$ (for details see [14, Section 3.3]). Since these equations hold pointwise in $x$, we here refer to these equations as the strong equations (in distinction of the weak equations obtained by testing and integrating; see Section 3.6). Regarding the brackets $\langle.,\rangle.(x)$ in (2.18) as a dual pairing, the operator $\Delta$ is a mapping to the dual jets,

$$
\Delta: \mathfrak{J}^{\text {test }} \rightarrow\left(\mathfrak{J}^{\text {test }}\right)^{*}
$$

The corresponding inhomogeneous equation arises for example in the perturbation expansion [14]. It reads

$$
\langle\mathfrak{u}, \Delta \mathfrak{v}\rangle=\langle\mathfrak{u}, \mathfrak{w}\rangle \quad \text { for all } \mathfrak{u} \in \mathfrak{J}^{\text {test }},
$$

where $\mathfrak{w} \in\left(\mathfrak{J}^{\text {test }}\right)^{*}$ is a given inhomogeneity. In order to avoid confusion, we point out that this equation is again evaluated pointwise for $x \in M$, and therefore we refer to it as the strong linearized field equations. For brevity, sometimes we leave out the pointwise testing and write this equation in the shorter form (1.1).

In 21] higher $\tau$-derivatives of (2.16) are computed. Here we only need the operator $\Delta_{2}: \mathfrak{J}^{\text {test }} \times \mathfrak{J}^{\text {test }} \rightarrow \mathfrak{J}^{*}$ defined by

$$
\begin{aligned}
& \left\langle\mathfrak{u}, \Delta_{2}\left[\mathfrak{v}_{1}, \mathfrak{v}_{2}\right]\right\rangle(x) \\
& \quad=\frac{1}{2} \nabla_{\mathfrak{u}}\left(\int_{M}\left(\nabla_{1, \mathfrak{v}_{1}}+\nabla_{2, \mathfrak{v}_{1}}\right)\left(\nabla_{1, \mathfrak{v}_{2}}+\nabla_{2, \mathfrak{v}_{2}}\right) \mathcal{L}(x, y) d \rho(y)-\nabla_{\mathfrak{v}_{1}} \nabla_{\mathfrak{v}_{2}} \mathfrak{s}\right) .
\end{aligned}
$$

Here we always use the convention that the "partial jet derivatives" do not act on jets contained in other derivatives, so that for example

$$
\left(\nabla_{\mathfrak{v}_{1}} \nabla_{\mathfrak{v}_{2}} \mathfrak{s}\right)(x)=b_{1}(x) b_{2}(x) \mathfrak{s}
$$

where $b_{1}$ and $b_{2}$ denote the scalar components of $\mathfrak{v}_{1}$ and $\mathfrak{v}_{2}$, respectively. 
2.4. Surface Layer Integrals. Surface layer integrals were first introduced in [19] as double integrals of the general form

$$
\int_{\Omega}\left(\int_{M \backslash \Omega}(\cdots) \mathcal{L}(x, y) d \rho(y)\right) d \rho(x)
$$

where $(\cdots)$ stands for a suitable differential operator formed of jets. A surface layer integral generalizes the concept of a surface integral over $\partial \Omega$ to the setting of causal fermion systems. The connection can be understood most easily in the case when $\mathcal{L}(x, y)$ vanishes unless $x$ and $y$ are close together. In this case, we only get a contribution to (2.20) if both $x$ and $y$ are close to the boundary of $\Omega$. A more detailed explanation of the idea of a surface layer integrals is given in [19, Section 2.3].

In [19, 20, 21], conservation laws for surface layer integrals were derived. The statement is that if $\mathfrak{v}$ describes a symmetry of the system or if $\mathfrak{v}$ satisfies the linearized field equations, then suitable surface layer integrals (2.20) vanish for every compact $\Omega \subset M$. The significance of these conservation laws for our problem lies in the fact that it $\mathfrak{v}$ is not a solution of the linearized field equations, then the surface layer integral still is conserved approximately in the sense that its change in time can be controlled by $\Delta \mathfrak{v}$. For this reason, these surface layer integrals are very useful for getting estimates, which we refer to as energy estimates. More specifically, the following surface layer integrals are important for developing energy estimates and will (in a slightly modified form) play a crucial role in our analysis: The symplectic form $\sigma^{\Omega}$ defined by (for details see [20, Section 4.3])

$$
\sigma^{\Omega}(\mathfrak{u}, \mathfrak{v}):=\int_{\Omega} d \rho(x) \int_{M \backslash \Omega} d \rho(y)\left(\nabla_{1, \mathfrak{u}} \nabla_{2, \mathfrak{v}}-\nabla_{1, \mathfrak{v}} \nabla_{2, \mathfrak{u}}\right) \mathcal{L}(x, y)
$$

and the surface layer inner product $(\mathfrak{u}, \mathfrak{v})^{\Omega}$ (for details see [21, Theorem 1.1 and Corollary 3.11])

$$
(\mathfrak{u}, \mathfrak{v})^{\Omega}:=\int_{\Omega} d \rho(x) \int_{M \backslash \Omega} d \rho(y)\left(\nabla_{1, \mathfrak{u}} \nabla_{1, \mathfrak{v}}-\nabla_{2, \mathfrak{u}} \nabla_{2, \mathfrak{v}}\right) \mathcal{L}(x, y) .
$$

In [13], these surface layer integrals were computed for Dirac systems in the presence of an electromagnetic potential in Minkowski space.

2.5. Positive Functionals Arising from Second Variations. For the alternative energy estimates of Section 3.3 we will work with positive functionals which arise in the analysis of second variations [12. We now recall a few concepts and results (the reader who prefers to work with the energy estimates of Section 3.2 may skip this section).

Clearly, if $\rho$ is a minimizing measure, then second variations are non-negative. For our purposes, it again suffices to consider variations of the form (2.14), where for simplicity we assume that $f_{\tau}$ and $F_{\tau}$ are trivial outside a compact set. Under these assumptions, it is proven in [12, Theorem 1.1] that

$$
\int_{M} d \rho(x) \int_{M} d \rho(y) \nabla_{1, \mathfrak{v}} \nabla_{2, \mathfrak{v}} \mathcal{L}(x, y)+\int_{M} \nabla^{2} \ell_{x}(\mathfrak{v}, \mathfrak{v}) d \rho(x) \geq 0,
$$

where jet $\mathfrak{v}$ is again the infinitesimal generator of the variation (2.17). For our purposes, it is preferable to write this inequality as

$$
\frac{1}{2} \int_{M} d \rho(x) \int_{M} d \rho(y)\left(\nabla_{1, \mathfrak{v}}+\nabla_{2, \mathfrak{v}}\right)^{2} \mathcal{L}(x, y)-\left(\nabla^{2} \mathfrak{s}\right)(\mathfrak{v}, \mathfrak{v}) \geq 0 .
$$




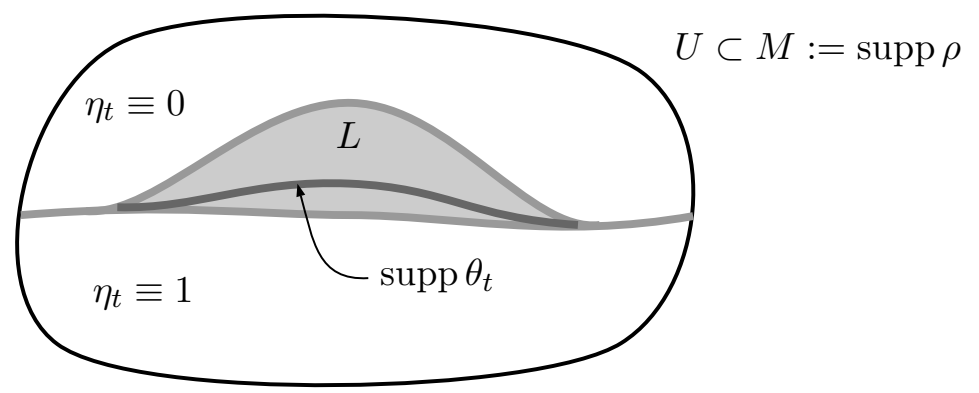

Figure 1. A local foliation.

Then it is obvious that the integrals are well-defined if we assume that $\mathfrak{u}, \mathfrak{v} \in \mathfrak{J}^{2}$ (see Definition 2.1). Moreover, using (2.3) and (2.18), the inequality can be written in the compact form

$$
\langle\mathfrak{v}, \Delta \mathfrak{v}\rangle_{M} \geq 0
$$

where we used the notation

$$
\langle\mathfrak{u}, \Delta \mathfrak{v}\rangle_{M}:=\int_{M}\langle\mathfrak{u}, \Delta \mathfrak{v}\rangle(x) d \rho(x) .
$$

In other words, the operator $\Delta$ is positive semi-definite. This might come as a surprise, because the analogous inequality for the wave operator in Minkowski space is violated. Instead, this inequality holds (up to an irrelevant sign) for the Laplacian in the Riemannian setting. These facts are not a contradiction if one keeps in mind that the operator $\Delta$ has a structure which is very different from a PDE. The basic reason why (2.24) holds is that, in the setting of causal variational principles, we consider minimizers. In contrast, the Dirichlet energy in the hyperbolic setting is unbounded from below, making it necessary to work merely with critical points.

\section{Hyperbolic Subsets of Space-Time}

3.1. Local Foliations by Surface Layers. Following the procedure for hyperbolic partial differential equations, our first goal is to analyze the initial value problem "locally" in an open subset $U$ of space-time $M$. In analogy to the usual procedure of choosing a local time function $t$ (like for example the time coordinate of a local observer) and considering the foliation by the hypersurfaces $t=$ const, we here want to choose a foliation of a compact subset $L \subset U$ by surface layers. This motivates the following definition.

Definition 3.1. Let $U \subset M$ be an open subset of space-time and $I \subset \mathbb{R}$ a compact interval. Moreover, we let $\eta \in C^{\infty}(I \times U, \mathbb{R})$ be a function with $0 \leq \eta \leq 1$ which for all $t \in I$ has the following properties:

(i) The function $\theta(t,):.=\partial_{t} \eta(t,$.$) is non-negative and compactly supported in U$.

(ii) For all $x \in \operatorname{supp} \theta(t,$.$) and all y \in M \backslash U$, the function $\mathcal{L}(x, y)$ as well as its first and second derivatives in the direction of $\mathfrak{J}_{0}^{\text {test }}$ vanish.

We also write $\eta(t, x)$ as $\eta_{t}(x)$ and $\theta(t, x)$ as $\theta_{t}(x)$. We refer to $\left(\eta_{t}\right)_{t \in I}$ as a local foliation inside $U$.

The situation in mind is shown in Figure 1. The parameter $t$ can be thought of as the time of a local observer and will often simply be referred to as time. The support of the function $\theta_{t}$ is a surface layer. The function $\eta_{t}$ should be thought of as being 
equal to one in the past and equal to zero in the future of this surface layer (where the distinction between future and past will become clear later; see the last paragraph of Section 3.4 below). The condition (i) implies that the set $L$ defined by

$$
L:=\bigcup_{t \in I} \operatorname{supp} \theta_{t}
$$

is compact. It is the region of space-time described by the local foliation. The condition (ii) has the purpose to ensure that the dynamics in the region $L$ does not depend on the jets outside $U$, making it possible to restrict attention to the space-time region $U$. Sometimes, we refer to this property that $L$ is $\mathcal{L}$-localized in $U$. One way of satisfying (ii) is to simply choose $U=M$. However, in the applications it may be desirable to "localize" the problem for example by choosing $U$ as the domain of a coordinate chart. In applications when $\mathcal{L}(x, y)$ is of short range (as introduced in [20, Section 2.3]), the condition (ii) can be arranged easily by choosing $U$ to be relatively compact and sufficiently large. When constructing global solutions, it will be useful to assume that $U$ is relatively compact (see Definition 4.7 in Section 4.3).

For the following constructions, it will be useful to combine the functions $\eta_{t}$ and $\theta_{t}$ with the measure $\rho$ such as to form new measures: The measure

$$
d \rho_{t}(x):=\theta_{t}(x) d \rho(x)
$$

with $t \in I$ is supported in the surface layer at time $t$. Likewise, the measures

$$
\eta_{t} d \rho \quad \text { and } \quad\left(1-\eta_{t}\right) d \rho
$$

are supported in the past respectively future of the surface layer at time $t$. For the measures supported in a space-time strip, we use the notation

$$
\eta_{\left[t_{0}, t_{1}\right]} d \rho \quad \text { with } \quad \eta_{\left[t_{0}, t_{1}\right]}:=\eta_{t_{1}}-\eta_{t_{0}} \in C_{0}^{\infty}(U),
$$

where we always choose $t_{0}, t_{1} \in I$ with $t_{0} \leq t_{1}$. Note that the function $\eta_{\left[t_{0}, t_{1}\right]}$ is supported in $L$.

3.2. Energy Estimates Using the Surface Layer Inner Product. For the analysis of local foliations we shall make use of class of surface layer integral, which we now introduce. In preparation, we need to specify the class of jets to work with. In order to have differentiability and regularity properties, it is a good idea to restrict attention to test jets. But, depending on the application, it might be necessary to restrict the jet space even further (the crucial point is that one must satisfy the hyperbolicity conditions in Definition 3.3 below). In order to have the largest possible flexibility, we shall work with a subspace

$$
\mathfrak{J}^{\text {vary }} \subset \mathfrak{J}^{\text {test }}
$$

which we can choose arbitrarily (in particular, the scalar component of $\mathfrak{J}^{\text {vary }}$ does not need to be nontrivial in the sense (2.8); this will be discussed in Example6.1 at the end of this paper). Similar to (2.12), $\mathfrak{J}_{0}^{\text {vary }}$ denotes the compactly supported jets in $\mathfrak{J}^{\text {vary }}$. We let $\mathfrak{J}_{U}$ be the restriction of these jets to $U$,

$$
\mathfrak{J}_{U}:=\left\{\left.\mathfrak{u}\right|_{U} \mid \mathfrak{u} \in \mathfrak{J}_{0}^{\text {vary }}\right\} .
$$


For any $t \in I$ we introduce the bilinear form

$$
\begin{aligned}
I_{2}^{t}(., .): \mathfrak{J}_{U} \times \mathfrak{J}_{U} & \rightarrow \mathbb{R} \\
I_{2}^{t}(\mathfrak{u}, \mathfrak{v})=\int_{U} d \rho(x) & \eta_{t}(x) \int_{U} d \rho(y)\left(1-\eta_{t}(y)\right) \\
\times & \left(\nabla_{1, \mathfrak{u}}-\nabla_{2, \mathfrak{u}}\right)\left(\nabla_{1, \mathfrak{v}}+\nabla_{2, \mathfrak{v}}\right) \mathcal{L}(x, y) .
\end{aligned}
$$

In order to ensure that the integrals are well-defined, we assume throughout this section that $\mathfrak{J}^{\text {test }}$ is surface layer regular (see Definition 2.2). Symmetrizing and antisymmetrizing gives the bilinear forms

$$
\begin{aligned}
(\mathfrak{u}, \mathfrak{v})^{t} & =\frac{1}{2}\left(I_{2}^{t}(\mathfrak{u}, \mathfrak{v})+I_{2}^{t}(\mathfrak{v}, \mathfrak{u})\right) \\
& =\int_{U} d \rho(x) \eta_{t}(x) \int_{U} d \rho(y)\left(1-\eta_{t}(y)\right)\left(\nabla_{1, \mathfrak{u}} \nabla_{1, \mathfrak{v}}-\nabla_{2, \mathfrak{u}} \nabla_{2, \mathfrak{v}}\right) \mathcal{L}(x, y) \\
\sigma^{t}(\mathfrak{u}, \mathfrak{v}) & =\frac{1}{2}\left(I_{2}^{t}(\mathfrak{u}, \mathfrak{v})-I_{2}^{t}(\mathfrak{v}, \mathfrak{u})\right) \\
& =\int_{U} d \rho(x) \eta_{t}(x) \int_{U} d \rho(y)\left(1-\eta_{t}(y)\right)\left(\nabla_{1, \mathfrak{u}} \nabla_{2, \mathfrak{v}}-\nabla_{1, \mathfrak{v}} \nabla_{2, \mathfrak{u}}\right) \mathcal{L}(x, y),
\end{aligned}
$$

referred to as the surface layer inner product and the symplectic form, respectively. These surface layer integrals are "softened versions" of the surface layer integrals (2.22) and (2.21) mentioned in Section 2.4, where the characteristic functions $\chi_{\Omega}$ and $\chi_{M \backslash \Omega}$ are replaced by the smooth cutoff functions $\eta_{t}$ and $1-\eta_{t}$, respectively.

The quantity $(\mathfrak{u}, \mathfrak{u})^{t}$ will be of central importance in the following constructions. It plays the role of the energy used in our energy estimates (for its physical interpretation see the paragraph after Definition [3.3). In preparation of these estimates, we derive an energy identity:

Lemma 3.2. (energy identity) For any jet $\mathfrak{v}=(b, v) \in \mathfrak{J}_{U}$,

$$
\begin{aligned}
\frac{d}{d t}(\mathfrak{v}, \mathfrak{v})^{t}= & 2 \int_{U}\langle\mathfrak{v}, \Delta \mathfrak{v}\rangle(x) d \rho_{t}(x) \\
& -2 \int_{U} \Delta_{2}[\mathfrak{v}, \mathfrak{v}] d \rho_{t}(x)+\mathfrak{s} \int_{U} b(x)^{2} d \rho_{t}(x) .
\end{aligned}
$$

Proof. We first derive the identity by a formal computation and give the analytic justification afterward. Differentiating (3.6) with respect to $t$ gives

$$
\begin{aligned}
\frac{d}{d t}(\mathfrak{v}, \mathfrak{v})^{t}= & \int_{U} d \rho(x) \theta_{t}(x) \int_{U} d \rho(y)\left(1-\eta_{t}(y)\right)\left(\nabla_{1, \mathfrak{v}}^{2}-\nabla_{2, \mathfrak{v}}^{2}\right) \mathcal{L}(x, y) \\
& -\int_{U} d \rho(x) \eta_{t}(x) \int_{U} d \rho(y) \theta_{t}(y)\left(\nabla_{1, \mathfrak{v}}^{2}-\nabla_{2, \mathfrak{v}}^{2}\right) \mathcal{L}(x, y) \\
= & \int_{U} d \rho(x) \theta_{t}(x) \int_{U} d \rho(y)\left(\nabla_{1, \mathfrak{v}}^{2}-\nabla_{2, \mathfrak{v}}^{2}\right) \mathcal{L}(x, y) .
\end{aligned}
$$

Next, for all $x \in L$ we may use Definition 3.1 (ii) to change the integration range in (2.18) from $M$ to $U$,

$$
\langle\mathfrak{v}, \Delta \mathfrak{v}\rangle(x)=\int_{U} \nabla_{1, \mathfrak{v}}\left(\nabla_{1, \mathfrak{v}}+\nabla_{2, \mathfrak{v}}\right) \mathcal{L}(x, y) d \rho(y)-\mathfrak{s} b(x)^{2} .
$$


Multiplying by $\theta_{t}$ and integrating, we obtain

$$
\begin{aligned}
0= & \int_{U} \theta_{t}(x)\langle\mathfrak{v}, \Delta \mathfrak{v}\rangle(x) d \rho(x)+\mathfrak{s} \int_{U} \theta_{t}(x) b(x)^{2} d \rho(x) \\
& -\int_{U} d \rho(x) \theta_{t}(x) \int_{U} d \rho(y)\left(\nabla_{1, \mathfrak{v}}^{2}+\nabla_{1, \mathfrak{v}} \nabla_{2, \mathfrak{v}}\right) \mathcal{L}(x, y) .
\end{aligned}
$$

We multiply this equation by two and add (3.9). This gives

$$
\begin{aligned}
\frac{d}{d t}(\mathfrak{v}, \mathfrak{v})^{t}= & -\int_{U} d \rho(x) \theta_{t}(x) \int_{U} d \rho(y)\left(\nabla_{1, \mathfrak{v}}+\nabla_{2, \mathfrak{v}}\right)^{2} \mathcal{L}(x, y) \\
& +2 \int_{U} \theta_{t}(x)\langle\mathfrak{v}, \Delta \mathfrak{v}\rangle(x) d \rho(x)+2 \mathfrak{s} \int_{U} \theta_{t}(x) b(x)^{2} d \rho(x) .
\end{aligned}
$$

Using the property in Definition 3.1(ii), in the $y$-integral we may replace the integration range $U$ by $M$, making it possible to apply (2.19). Rewriting the obtained integrals using the notation (3.2) gives (3.8).

It remains to give a rigorous justification of taking the time derivative of (3.6). To this end, we first take the difference quotient and rewrite it as

$$
\begin{aligned}
& \frac{1}{\Delta t}\left((\mathfrak{v}, \mathfrak{v})^{t+\Delta t}-(\mathfrak{v}, \mathfrak{v})^{t}\right) \\
& =\int_{U} d \rho(x) \frac{\eta_{t+\Delta t}(x)-\eta_{t}(x)}{\Delta t} \int_{U} d \rho(y)\left(\nabla_{1, \mathfrak{v}} \nabla_{1, \mathfrak{v}}-\nabla_{2, \mathfrak{v}} \nabla_{2, \mathfrak{v}}\right) \mathcal{L}(x, y) \\
& \quad-\int_{U} d \rho(x) \frac{\left(\eta_{t+\Delta t}-\eta_{t}\right)(x)}{\Delta t} \int_{U} d \rho(y)\left(\eta_{t+\Delta t}+\eta_{t}\right)(y)\left(\nabla_{1, \mathfrak{v}} \nabla_{1, \mathfrak{v}}-\nabla_{2, \mathfrak{v}} \nabla_{2, \mathfrak{v}}\right) \mathcal{L}(x, y)
\end{aligned}
$$

Since $\mathfrak{J}^{\text {test }}$ is assumed to be surface layer regular, we know from Definition 2.2 (ii) and Definition 2.1 (ii) (both evaluated for $p=2$ ) that the above jet derivatives exist and are in $\left.L_{\mathrm{loc}}^{\infty}\left(L^{1} M, d \rho(y)\right), d \rho(x)\right)$. Therefore, the above $y$-integrals can be all be bounded uniformly in $\Delta t$ by the function

$$
2 \int_{M}\left|\left(\nabla_{1, \mathfrak{v}} \nabla_{1, \mathfrak{v}}-\nabla_{2, \mathfrak{v}} \nabla_{2, \mathfrak{v}}\right) \mathcal{L}(x, y)\right| d \rho(y) \in L_{\mathrm{loc}}^{1}(M, d \rho) .
$$

Clearly, the factor $\eta_{t+\Delta t}+\eta_{t}$ converges pointwise to $2 \eta_{t}$. Moreover, the difference quotient $\left(\eta_{t+\Delta t}-\eta_{t}\right) / \Delta t$ has uniformly compact support and converges pointwise to $\theta_{t}$. Therefore, we can take the limit $\Delta t \rightarrow 0$ with the help of Lebesgue's dominated convergence theorem.

In order to make use of this energy identity, we need to impose a condition which we call hyperbolicity condition. This notion can be understood as follows. In the theory of hyperbolic partial differential equations, the hyperbolicity of the equations (as expressed for example by the notions of normally hyperbolic operators or symmetric hyperbolic systems) gives rise to a positive energy. In our setting, we clearly have no partial differential equation. Instead, we take a positivity condition for the energy to define hyperbolicity. As we shall see, this condition is precisely what is needed in order to obtain existence and uniqueness of solutions. We first define the hyperbolicity condition and explain it afterward.

For all $x \in M$ we choose the subspace of the tangent space spanned by the test jets,

$$
\Gamma_{x}:=\left\{u(x) \mid u \in \Gamma^{\text {test }}\right\} \subset T_{x} \mathcal{F} .
$$


We introduce a Riemannian metric $g_{x}$ on $\Gamma_{x}$. This Riemannian metric also induces a pointwise scalar product on the jets. Namely, setting

$$
\mathfrak{J}_{x}:=\mathbb{R} \oplus \Gamma_{x}
$$

we obtain the scalar product on $\mathfrak{J}_{x}$

$$
\langle., .\rangle_{x}: \mathfrak{J}_{x} \times \mathfrak{J}_{x} \rightarrow \mathbb{R}, \quad\langle\mathfrak{v}, \tilde{\mathfrak{v}}\rangle_{x}:=b(x) \tilde{b}(x)+g_{x}(v(x), \tilde{v}(x))
$$

We denote the corresponding norm by $\|\cdot\|_{x}$.

Definition 3.3. The local foliation $\left(\eta_{t}\right)_{t \in I}$ inside $U$ satisfies the hyperbolicity condition if there is a constant $C>0$ such that for all $t \in I$,

$$
(\mathfrak{v}, \mathfrak{v})^{t} \geq \frac{1}{C^{2}} \int_{U}\left(\|\mathfrak{v}(x)\|_{x}^{2}+\left|\Delta_{2}[\mathfrak{v}, \mathfrak{v}]\right|\right) d \rho_{t}(x) \quad \text { for all } \mathfrak{v} \in \mathfrak{J}_{U}
$$

Let us explain the hyperbolicity condition. The inner product $(., .)^{t}$ was first introduced in [21] in a slightly different form where the smooth cutoff function $\eta_{t}$ is replaced by the characteristic function of a set $\Omega$. In [13] it was shown by longer explicit computations that for Dirac sea configurations in Minkowski space and choosing $\eta_{t}$ as a characteristic function being identically equal to one in the past of the hypersurface $t=$ const, the inner product $(., .)^{t}$ reduces to a (positive definite) scalar product on Dirac wave functions and on the Maxwell field tensor. With this in mind, it is physically sensible to assume that $(\mathfrak{v}, \mathfrak{v})^{t}$ is positive.

The lower bound in (3.11) is a stronger and more quantitative version of positivity. Again for Dirac sea configurations in Minkowski space and for $\theta_{t}$ replaced by a characteristic function of the past of the surface $t=$ const, this inequality is satisfied in view of the explicit formulas in [13. In more general situations, the inequality (3.11) is not obvious and must be verified in all applications. More specifically, in the applications one can use the freedom in choosing the jet spaces $\mathfrak{J}^{\text {test }}$ and $\mathfrak{J}^{\text {vary }}$, the Riemannian metric in the scalar product (3.10) and the functions $\eta_{t}$ in order to arrange that (3.11) holds. Clearly, the smaller the jet space $\mathfrak{J}^{\text {vary }}$ is chosen, the easier it is to satisfy (3.11). The drawback is that the Cauchy problem will be solvable for more restrictive initial data (as will be made precise in Section 3.11).

We now explain how the above hyperbolicity condition can be used to derive energy estimates. We let $L$ be a lens-shaped region inside $U$ with the local foliation $\left.\left(\eta_{t}\right)_{t \in I}\right)$. We denote the norm corresponding to the jet scalar product by $\|\mathfrak{v}\|^{t}:=\sqrt{(\mathfrak{v}, \mathfrak{v})^{t}}$. We begin with a simple estimate of the energy identity in Lemma 3.2 ,

Lemma 3.4. Assume that the hyperbolicity condition of Definition 3.3 holds. Then for every $t \in I$ and all $\mathfrak{v} \in \mathfrak{J}_{U}$,

$$
\frac{d}{d t}\|\mathfrak{v}\|^{t} \leq C\|\Delta \mathfrak{v}\|_{L^{2}\left(U, d \rho_{t}\right)}+c\|\mathfrak{v}\|^{t}
$$

with

$$
c:=C^{2}+\frac{C^{2} \mathfrak{s}}{2}
$$


Proof. Applying (3.11) in (3.8), we obtain

$$
\begin{aligned}
\frac{d}{d t}(\mathfrak{v}, \mathfrak{v})^{t} & \leq 2 \int_{U}\langle\mathfrak{v}, \Delta \mathfrak{v}\rangle_{x} d \rho_{t}(x)-2 \int_{U} \Delta_{2}[\mathfrak{v}, \mathfrak{v}] d \rho_{t}(x)+\mathfrak{s} \int_{U} b(x)^{2} d \rho_{t}(x) \\
& \leq 2 \int_{U}\langle\mathfrak{v}, \Delta \mathfrak{v}\rangle_{x} d \rho_{t}(x)+\left(2 C^{2}+C^{2} \mathfrak{s}\right)(\mathfrak{v}, \mathfrak{v})^{t} \\
& \leq 2\|\mathfrak{v}\|_{L^{2}\left(U, d \rho_{t}\right)}\|\Delta \mathfrak{v}\|_{L^{2}\left(U, d \rho_{t}\right)}+2 c(\mathfrak{v}, \mathfrak{v})^{t} \\
& \leq 2 C\|\mathfrak{v}\|^{t}\|\Delta \mathfrak{v}\|_{L^{2}\left(U, d \rho_{t}\right)}+2 c(\mathfrak{v}, \mathfrak{v})^{t}
\end{aligned}
$$

where in the last line we applied (3.11). Using the relation $\partial_{t}\|\mathfrak{v}\|^{t}=\partial_{t}(\mathfrak{v}, \mathfrak{v})^{t} /\left(2\|\mathfrak{v}\|^{t}\right)$ gives the result.

Applying Grönwall-type estimates, the inequality (3.12) shows that $\|\mathfrak{v}\|^{t}$ grows at most exponentially in time, provided that $\Delta \mathfrak{v}$ decays in time sufficiently fast. We here make this statement precise by estimates in Hilbert spaces of jets with zero initial values. In the lens-shaped region $L$ we work with the $L^{2}$-scalar product

$$
\langle\mathfrak{u}, \mathfrak{v}\rangle_{L^{2}(L)}:=\int_{L}\langle\mathfrak{u}(x), \mathfrak{v}(x)\rangle_{x} \eta_{I}(x) d \rho(x),
$$

which, according to (3.2) and (3.3), can also be written in terms of a time integral,

$$
\langle\mathfrak{u}, \mathfrak{v}\rangle_{L^{2}(L)}=\int_{t_{0}}^{t_{\max }}\langle\mathfrak{u}, \mathfrak{v}\rangle_{L^{2}\left(U, d \rho_{t}\right)} d t
$$

The corresponding norm is denoted by $\|\cdot\|_{L^{2}(L)}$.

Proposition 3.5. (energy estimate) Assume that the hyperbolicity condition of Definition 3.3 holds. Then, choosing

$$
\Gamma=2 C e^{2 c\left(t_{\max }-t_{0}\right)}\left(t_{\max }-t_{0}\right),
$$

the following estimate holds,

$$
\|\mathfrak{v}\|_{L^{2}(L)} \leq \Gamma\|\Delta \mathfrak{v}\|_{L^{2}(L)} \quad \text { for all } \mathfrak{v} \in \mathfrak{J}_{U} \text { with }\|\mathfrak{v}\|^{t_{0}}=0
$$

Proof. We write the energy estimate of Lemma 3.4 as

$$
\frac{d}{d t}\left(e^{-2 c t}(\mathfrak{v}, \mathfrak{v})^{t}\right) \leq 2 e^{-2 c t} C\|\mathfrak{v}\|^{t}\|\Delta \mathfrak{v}\|_{L^{2}\left(U, d \rho_{t}\right)} .
$$

Integrating over $t$ from $t_{0}$ to some $t \in I$ and using the hyperbolicity condition (3.11), we obtain

$$
\begin{aligned}
e^{-2 c t}(\mathfrak{v}, \mathfrak{v})^{t} & =\int_{t_{0}}^{t} \frac{d}{d t^{\prime}}\left(e^{-2 c t^{\prime}}(\mathfrak{v}, \mathfrak{v})^{t^{\prime}}\right) d t^{\prime} \\
& \leq 2 C \int_{t_{0}}^{t} e^{-2 c t^{\prime}}\|\mathfrak{v}\|^{t^{\prime}}\|\Delta \mathfrak{v}\|_{L^{2}\left(U, d \rho_{t^{\prime}}\right)} d t^{\prime}
\end{aligned}
$$


Multiplying by $e^{2 c t}$ gives the inequality

$$
\begin{aligned}
(\mathfrak{v}, \mathfrak{v})^{t} & \leq 2 C \int_{t_{0}}^{t} e^{2 c\left(t-t^{\prime}\right)}\|\mathfrak{v}\|^{t^{\prime}}\|\Delta \mathfrak{v}\|_{L^{2}\left(U, d \rho_{t^{\prime}}\right)} d t^{\prime} \\
& \leq 2 C e^{2 c\left(t_{\max }-t_{0}\right)} \int_{t_{0}}^{t_{\max }}\|\mathfrak{v}\|^{t^{\prime}}\|\Delta \mathfrak{v}\|_{L^{2}\left(U, d \rho_{t^{\prime}}\right)} d t^{\prime} \\
& \leq 2 C e^{2 c\left(t_{\max }-t_{0}\right)}\|\Delta \mathfrak{v}\|_{L^{2}(L)}\left(\int_{t_{0}}^{t_{\max }}(\mathfrak{v}, \mathfrak{v})^{t^{\prime}} d t^{\prime}\right)^{\frac{1}{2}}
\end{aligned}
$$

where in the last step we used the Schwarz inequality and (3.14). Integrating once again over $t$ from $t_{0}$ to $t_{\max }$ gives

$$
\left(\int_{t_{0}}^{t_{\max }}(\mathfrak{v}, \mathfrak{v})^{t} d t\right)^{\frac{1}{2}} \leq 2 C e^{2 c\left(t_{\max }-t_{0}\right)}\left(t_{\max }-t_{0}\right)\|\Delta \mathfrak{v}\|_{L^{2}(L)} .
$$

Finally, we apply the hyperbolicity condition (3.11) in (3.14),

$$
\|v\|_{L^{2}(L)}=\left(\int_{t_{0}}^{t_{\max }}\|\mathfrak{v}\|_{L^{2}\left(U, d \rho_{t}\right)}^{2} d t\right)^{\frac{1}{2}} \leq C\left(\int_{t_{0}}^{t_{\max }}(\mathfrak{v}, \mathfrak{v})^{t} d t\right)^{\frac{1}{2}} .
$$

Combining this inequality with (3.16) gives the result.

3.3. Alternative Energy Estimates Using Second Variations. The energy estimates of the previous section were based on the hyperbolicity condition of Definition 3.3. Working with the surface layer inner product $(., .)^{t}$ has the advantage that it has a clear physical interpretation and significance (in particular, it gives rise to the scalar product of quantum theory [17]). Also, it can be verified in important examples that the hyperbolicity condition (3.11) is indeed satisfied. But one should keep in mind that the positivity of the surface layer inner product is a physical assumption which needs to be verified in all applications. From the mathematical point of view, it would be more convincing to work with quantities which are positive as a consequence of the mathematical structure of the causal variational principle. Such positive quantities were obtained in [12] by considering second variations (for basics see Section [2.5). We now show that positive quantities obtained from second variations can indeed be used for energy estimates, giving an alternative to the energy estimates in the previous section. The corresponding hyperbolicity condition (see Definition 3.7 below) is more natural from the mathematical point of view. The energy estimates in this section shed new light on the mathematical structure of causal variational principles. The reader who prefers to work with the surface layer inner product and the hyperbolicity condition of Definition 3.3 may skip this section.

Throughout this section, we assume that $\rho$ is a minimizing measure and that $\mathfrak{J}^{\text {test }}$ is surface layer regular (see Definition 2.2). Then, according to Definition 2.1 (ii) for $p=2$, we know that for all $\mathfrak{u}, \mathfrak{v} \in \mathfrak{J}^{\text {test }}$,

$$
\left(\nabla_{1, \mathfrak{u}}+\nabla_{2, \mathfrak{u}}\right)\left(\nabla_{1, \mathfrak{v}}+\nabla_{2, \mathfrak{v}}\right) \mathcal{L}(x, y) \in L_{\mathrm{loc}}^{\infty}\left(L^{1}(M, d \rho(y)), d \rho(x)\right) .
$$


Using that the function $\eta_{\left[t_{0}, t\right]}$ has compact support (see (3.3)), it follows that the following expression is well-defined,

$$
\begin{aligned}
& \int_{U} d \rho(x) \int_{U} d \rho(y)\left(\nabla_{1, \eta_{\left[t_{0}, t\right]} \mathfrak{u}}+\nabla_{2, \eta_{\left[t_{0}, t\right]} \mathfrak{u}}\right)\left(\nabla_{1, \eta_{\left[t_{0}, t\right]} \mathfrak{v}}+\nabla_{2, \eta_{\left[t_{0}, t\right]} \mathfrak{v}}\right) \mathcal{L}(x, y) \\
& -\left.2 \int_{U} \eta_{\left[t_{0}, t\right]}(x)^{2}\left(\nabla^{2} \mathfrak{s}\right)(\mathfrak{v}, \mathfrak{v})\right|_{x} d \rho(x) .
\end{aligned}
$$

Exactly as explained after (2.23), this expression can be written in the more compact form $\left\langle\eta_{\left[t_{0}, t\right]} \mathfrak{u}, \Delta\left(\eta_{\left[t_{0}, t\right]} \mathfrak{v}\right)\right\rangle_{M}$, giving rise to a bilinear form

$$
\langle\mathfrak{u}, \mathfrak{v}\rangle_{\left[t_{0}, t\right]}: \mathfrak{J}_{U} \times \mathfrak{J}_{U} \rightarrow \mathbb{R}, \quad\langle\mathfrak{u}, \mathfrak{v}\rangle_{\left[t_{0}, t\right]}:=\left\langle\eta_{\left[t_{0}, t\right]} \mathfrak{u}, \Delta\left(\eta_{\left[t_{0}, t\right]} \mathfrak{v}\right)\right\rangle_{M}
$$

According to (2.24), this inner product is positive semi-definite, i.e.

$$
\langle\mathfrak{v}, \mathfrak{v}\rangle_{\left[t_{0}, t\right]} \geq 0 \quad \text { for all } \mathfrak{v} \in \mathfrak{J}_{U}
$$

We denote the corresponding semi-norm by $\|\cdot\|_{\left[t_{0}, t\right]}$. Before going on, we point out that the jet $\eta_{\left[t_{0}, t\right]} \mathfrak{u}$ will in general not lie in $\mathfrak{J}^{2}$, because the condition (iii) in Definition 2.1 may be violated. Therefore, one should always keep in mind our jets are in $\mathfrak{J}_{U}$ only before multiplying by the cutoff function $\eta_{\left[t_{0}, t\right]}$.

We again begin with an energy identity.

Lemma 3.6. (energy identity) For all $\mathfrak{v} \in \mathfrak{J}_{U}$,

$$
\frac{d}{d t}\langle\mathfrak{v}, \mathfrak{v}\rangle_{\left[t_{0}, t\right]}=2\left\langle\eta_{\left[t_{0}, t\right]} \mathfrak{v}, \Delta\left(\theta_{t} \mathfrak{v}\right)\right\rangle_{M}
$$

(where we again used the notation (2.25) ).

Proof. The identity is obtained immediately by formally differentiating (3.18) and using the symmetry of the bilinear form $\langle., \Delta .\rangle_{M}$. Therefore, the only task is to justify the differentiation and the product rule. To this end, similar as in the proof of Lemma 3.2, we analyze the difference quotient in the limit $\Delta t \rightarrow 0$. In the last integral in (3.17), this is straightforward because the integrand converges pointwise and has uniformly compact support. Therefore, it remains to consider the first line in (3.17) for $\mathfrak{u}=\mathfrak{v}$. Using the symmetry in the arguments $x$ and $y$, we can write the difference quotient as

$$
\begin{array}{rl}
2 \int_{U} & d \rho(x) \int_{U} d \rho(y) \frac{1}{\Delta t}\left(\nabla_{1, \eta_{\left[t_{0}, t+\Delta t\right]} \mathfrak{v}}-\nabla_{1, \eta_{\left[t_{0}, t\right]} \mathfrak{v}}\right) \\
& \times\left(\left(\nabla_{1, \eta_{\left[t_{0}, t+\Delta t\right]} \mathfrak{v}}+\nabla_{2, \eta_{\left[t_{0}, t+\Delta t\right]} \mathfrak{v}}\right)+\left(\nabla_{1, \eta_{\left[t_{0}, t\right]} \mathfrak{v}}+\nabla_{2, \eta_{\left[t_{0}, t\right]} \mathfrak{v}}\right)\right) \mathcal{L}(x, y) .
\end{array}
$$

Since $\mathfrak{J}^{\text {test }}$ is surface layer regular, we know from Definition 2.2 (i) that the derivatives exist. Moreover, the $y$-integral can be estimated uniformly in $\Delta t$ by

$$
2 \int_{M}\left|\nabla_{1, \mathfrak{v}}\left(\nabla_{1, \mathfrak{v}}+\nabla_{2, \mathfrak{v}}\right) \mathcal{L}(x, y)\right| d \rho(y) \in L_{\mathrm{loc}}^{1}(M, d \rho) .
$$

Now we can take the limit $\Delta t \rightarrow 0$ exactly as in the proof of Lemma 3.2 .

Definition 3.7. The local foliation $\left(\eta_{t}\right)_{t \in I=\left[t_{0}, t_{\max }\right]}$ inside $U$ satisfies the alternative hyperbolicity condition if there exists a constant $C>0$ and $\underline{t} \in I$ such that, for 
all $t \in\left[\underline{t}, t_{\max }\right]$ and all $\mathfrak{v} \in \mathfrak{J}_{U}$

$$
\begin{aligned}
\langle\mathfrak{v}, \mathfrak{v}\rangle_{\left[t_{0}, t\right]} & \geq \frac{1}{C^{2}} \int_{U} \eta_{\left[t_{0}, t\right]}(x)\langle\mathfrak{v}(x), \mathfrak{v}(x)\rangle_{x} d \rho(x) \\
\left|\left\langle\eta_{\left[t_{0}, t\right]} \mathfrak{v}, \Delta\left(\theta_{t} \mathfrak{v}\right)\right\rangle_{M}\right| & \leq C\left(\|\mathfrak{v}\|_{\left[t_{0}, t\right]}^{2}+\left|\left\langle\eta_{\left[t_{0}, t\right]} \mathfrak{v}, \Delta\left(\left(1-\eta_{\left[t_{0}, t\right]}\right) \mathfrak{v}\right)\right\rangle_{M}\right|\right) .
\end{aligned}
$$

We now explain these conditions and compare them to the previous hyperbolicity condition of Definition 3.3. Both inequalities (3.21) and (3.22) strengthen and quantify the positivity property (3.19). The explicit computations in [13, Section 6] show that these inequalities are satisfied for Dirac sea configurations in Minkowski space in the presence of Dirac currents and a Maxwell field. Compared to the hyperbolicity condition in Definition [3.3, there are several major structural differences: First, as already mentioned at the beginning of this section, the positivity (3.19) is not a physical assumption, but it follows already from the structure of the causal variational principle. Second, in contrast to (3.11), the energy identity (3.20) and consequently also the inequalities (3.21) and (3.22) do not involve the quadratic correction $\Delta_{2}$ to the linearized field equations. This is remarkable because it means that we do not need to control the nonlinear corrections in the energy estimates. A third difference is that, in contrast to the surface layer integral $(., .)^{t}$, the energy $\langle., .\rangle_{\left[t_{0}, t\right]}$ in (3.19) involves an integral over the time strip $\left[t_{0}, t\right]$. As a consequence, this inner product typically tends to zero in the limit $t \rightarrow t_{0}$, making it difficult to satisfy the inequalities (3.21) and (3.22). This is the reason why in Definition 3.7 we merely assume that that these inequalities hold for all $t \in\left[\underline{t}, t_{\mathrm{max}}\right]$. We finally remark that, in contrast to $(., .)^{t}$, the energy $\langle., .\rangle_{\left[t_{0}, t\right]}$ does not distinguish a direction of time; this will be discussed further in Section 3.4 below.

By combining the above energy identity with the hyperbolicity condition, we now derive energy estimates.

Lemma 3.8. Assume that the alternative hyperbolicity condition of Definition 3.7 holds. Then for every $t \in\left[\underline{t}, t_{\max }\right]$ and all $\mathfrak{v} \in \mathfrak{J}_{U}$,

$$
\frac{d}{d t}\|\mathfrak{v}\|_{\left[t_{0}, t\right]} \leq C^{2}\|\Delta \mathfrak{v}\|_{L^{2}(L)}+c\|\mathfrak{v}\|_{\left[t_{0}, t\right]},
$$

where $c=2 C$.

Proof. We estimate (3.20) using (3.22) to obtain

$$
\begin{aligned}
\left|\frac{d}{d t}\langle\mathfrak{v}, \mathfrak{v}\rangle_{\left[t_{0}, t\right]}\right| & \leq 2 C\left(\|\mathfrak{v}\|_{\left[t_{0}, t\right]}^{2}+\mid\left\langle\eta_{\left[t_{0}, t\right]} \mathfrak{v}, \Delta\left(\left(1-\eta_{\left[t_{0}, t\right]}\right) \mathfrak{v}\right)\right\rangle_{M}\right) \\
& \leq 4 C\|\mathfrak{v}\|_{\left[t_{0}, t\right]}^{2}+2 C\left|\left\langle\eta_{\left[t_{0}, t\right]} \mathfrak{v}, \Delta \mathfrak{v}\right\rangle_{M}\right| \\
& \left.=4 C\|\mathfrak{v}\|_{\left[t_{0}, t\right]}^{2}+2 C \mid\langle\mathfrak{v}, \Delta \mathfrak{v}\rangle_{L^{2}\left(L, d \bar{\rho}_{t_{\text {min }}}^{t}\right)}\right) \mid .
\end{aligned}
$$

Applying the Schwarz inequality as well as (3.21) gives

$$
\begin{aligned}
\left|\frac{d}{d t}\langle\mathfrak{v}, \mathfrak{v}\rangle_{\left[t_{0}, t\right]}\right| & \left.\leq 4 C\|\mathfrak{v}\|_{\left[t_{0}, t\right]}^{2}+2 C\|\mathfrak{v}\|_{L^{2}\left(L,{ }_{\bar{\rho}}^{t}\right.}^{t}\right) \\
& \leq 4 C\|\mathfrak{v}\|_{\left[t_{\text {min }}, t\right]}^{2}+2 C^{2}\|\mathfrak{v}\|_{\left[t_{0}, t\right]}\|\Delta \mathfrak{v}\|_{L^{2}(L)} .
\end{aligned}
$$

Using the relation $\partial_{t}\|\mathfrak{v}\|_{\left[t_{0}, t\right]}=\partial_{t}\langle\mathfrak{v}, \mathfrak{v}\rangle_{\left[t_{0}, t\right]} /\left(2\|\mathfrak{v}\|_{\left[t_{0}, t\right]}\right)$ gives the result. 
Proposition 3.9. (energy estimate) Assume that the alternative hyperbolicity condition of Definition 3.7 holds. Then, choosing

$$
\Gamma=\frac{C^{2}}{c}\left(e^{c\left(t_{\max }-\underline{t}\right)}-1\right)
$$

the following estimate holds,

$$
\|\mathfrak{v}\|_{L^{2}(L)} \leq \Gamma\|\Delta \mathfrak{v}\|_{L^{2}(L)} \quad \text { for all } \mathfrak{v} \in \mathfrak{J}_{U} \text { with }\|\mathfrak{v}\|_{\left[t_{0}, t\right]}=0 .
$$

Proof. We write the energy estimate of Lemma 3.8 as

$$
\frac{d}{d t}\left(e^{-c t}\|\mathfrak{v}\|_{\left[t_{0}, t\right]}\right) \leq C^{2} e^{-c t}\|\Delta \mathfrak{v}\|_{L^{2}(L)} .
$$

Integrating from $\underline{t}$ to $t_{\max }$ and using that the initial data vanishes gives

$$
e^{-c t_{\max }}\|\mathfrak{v}\|_{I} \leq \frac{C^{2}}{c}\left(e^{-c \underline{t}}-e^{-c t_{\max }}\right)\|\Delta \mathfrak{v}\|_{L^{2}(L)} .
$$

Multiplying by $e^{c t_{\max }}$ gives the result.

We finally motivate the hyperbolicity conditions of Definition 3.7 and clarify the connection between the norms $\|\mathfrak{v}\|_{I}$ and $\|\mathfrak{v}\|_{L^{2}(L)}$. We first show that, under general assumptions on the Lagrangian, the norm $\|\mathfrak{v}\|_{I}$ can be estimated from above by the $L^{2}$-norm.

Lemma 3.10. Assume that the Lagrangian satisfies the condition

$$
C_{\mathcal{L}}^{2}:=\left\|\eta_{I} \nabla^{2} \ell\right\|_{L^{\infty}(L)}+\sup _{x \in M}\left\|\nabla_{1} \nabla_{2} \mathcal{L}(x, y)\right\|_{L^{1}(L)}<\infty
$$

(where, similar to the notation in (3.13), $L^{2}(L)$ refers to the measure $\eta_{I} d \rho$ ). Then for any $\mathfrak{v} \in \mathfrak{J}_{U}$,

$$
\|\mathfrak{v}\|_{I} \leq C_{\mathcal{L}}\|\mathfrak{v}\|_{L^{2}(L)} .
$$

Proof. We first note that, according to (2.25),

$$
\begin{aligned}
\langle\mathfrak{v}, \mathfrak{v}\rangle_{I}= & \left\langle\eta_{I} \mathfrak{v}, \Delta\left(\eta_{I} \mathfrak{v}\right)\right\rangle_{M} \\
= & \int_{M} d \rho(x) \nabla_{\eta_{I} \mathfrak{v}}\left(\int_{M}\left(\nabla_{1, \eta_{I} \mathfrak{v}}+\nabla_{2, \eta_{I}}\right) \mathcal{L}(x, y) d \rho(y)-\nabla_{\eta_{I} \mathfrak{v}}\right) \\
= & \int_{M} \eta_{I}^{2}(x) \nabla_{\mathfrak{v}}^{2} \ell(x) d \rho(x) \\
& \quad+\int_{M} \eta_{I}(x) d \rho(x) \int_{M} \eta_{I}(y) d \rho(y) \nabla_{1, \mathfrak{v}} \nabla_{2, \mathfrak{v}} \mathcal{L}(x, y) .
\end{aligned}
$$

We estimate the first integral by

$$
\left|\int_{M} \eta_{I}^{2}(x) \nabla_{\mathfrak{v}}^{2} \ell(x) d \rho(x)\right| \leq\|\mathfrak{v}\|_{L^{2}(L)}^{2}\left\|\eta_{I} \nabla^{2} \ell\right\|_{L^{\infty}(M)}
$$

The second integral, on the other hand, can be estimated by

$$
\begin{aligned}
& \left|\int_{M} \eta_{I}(x) d \rho(x) \int_{M} \eta_{I}(y) d \rho(y) \nabla_{1, \mathfrak{v}} \nabla_{2, \mathfrak{v}} \mathcal{L}(x, y)\right| \\
& \leq \int_{M} d \rho(x) \int_{M} d \rho(y) f(x, y) f(y, x) K(x, y)
\end{aligned}
$$

where we introduced the abbreviations

$$
f(x, y)=\sqrt{\eta_{I}(x)}\|\mathfrak{v}(x)\|_{x} \sqrt{\eta_{I}(y)} \quad \text { and } \quad K(x, y)=\left\|\nabla_{1} \nabla_{2} \mathcal{L}(x, y)\right\| .
$$


The last integral can be estimated as follows,

$$
\begin{aligned}
& \int_{M} d \rho(x) \int_{M} d \rho(y) f(x, y) f(y, x) K(x, y) \\
& \leq \frac{1}{2} \int_{M} d \rho(x) \int_{M} d \rho(y)\left(f(x, y)^{2}+f(y, x)^{2}\right) K(x, y) \\
& =\int_{M} d \rho(x) \int_{M} d \rho(y) f(x, y)^{2} K(x, y) \\
& \leq\left(\int_{M}\|\mathfrak{v}(x)\|_{x}^{2} \eta_{I}(x) d \rho(x)\right) \sup _{z \in M} \int_{M}|K(z, y)| \eta_{I}(y) d \rho(y) \\
& =\|\mathfrak{v}\|_{L^{2}(L)}^{2} \sup _{x}\|K(x, .)\|_{L^{1}(L)} \cdot
\end{aligned}
$$

Combining the terms gives the result.

We finally note that (3.21) is the converse inequality to (3.24). The inequality (3.21) in general does not hold for $L^{2}$-jets. The same is true for the inequality (3.22). This is why in Definition 3.7 we restrict attention to jets in $\mathfrak{J}_{U}$.

3.4. Lens-Shaped Regions and Time Orientation. We combine the previous concepts in the following useful notion:

Definition 3.11. A compact set $L \subset M$ is a lens-shaped region inside $U$ if there is a local foliation $\left(\eta_{t}\right)_{t \in I}$ inside $U$ satisfying (3.1) which satisfies the hyperbolicity conditions of either Definition 3.3 or Definition 3.7.

We now discuss the question of time orientability. A local foliation $\left(\eta_{t}\right)_{t \in I}$ distinguishes the future (the region where $\eta_{t} \equiv 0$ ) from the past (where $\eta_{t} \equiv 1$ ). But the time orientation was arbitrary; we could just as well have chosen a local foliation with the opposite time orientation. Indeed, the hyperbolicity condition of Definition 3.3 removes this arbitrariness, because it distinguishes a direction of time. In order to explain how this comes about, we note that changing the time direction corresponds to the replacement $\eta_{t} \rightarrow\left(1-\eta_{t}\right)$. In the above surface layer integrals, this corresponds to interchanging $x$ and $y$, which in (3.6) gives rise to a minus sign. Consequently, if we changed the time orientation, the inner product $(\mathfrak{v}, \mathfrak{v})^{t}$ in (3.11) would become negative. Therefore, a lens-shaped region which satisfies the hyperbolicity condition of Definition 3.3 always comes with a distinguished time orientation. If we assume that $M$ can be covered by lens-shaped regions (as is made precise by the notion of local hyperbolicity in Definition 4.1 below), we automatically obtain a global time orientation.

The alternative hyperbolicity condition of Definition 3.7, however, does not distinguish a time direction. Therefore, when working with this hyperbolicity condition, we must always assume that space-time can be oriented in the sense that we can distinguish lens-shaped regions with mutually compatible time directions. For brevity, we do not formalize this assumption.

3.5. The Cauchy Problem and Uniqueness of Strong Solutions. We want to study the Cauchy problem to the future (the solution to the future and past will be studied in Section 3.9 below). Therefore, we assume that we are given a local foliation with $I=\left[t_{0}, t_{\max }\right]$ of a lens-shaped region $L$ inside $U$, where $t_{0}$ and $t_{\max }$ are the initial and final times, respectively. 
In preparation of setting up the initial value problem, we need to specify what we mean by "v vanishes in the past of $t_{0} . "$ The obvious notion is to demand that $\mathfrak{v}$ vanishes identically in the region where $\eta_{t_{0}}$ is strictly positive, i.e. that $\eta_{t_{0}} \mathfrak{v} \equiv 0$. This condition is quite strong, because it also implies that $\mathfrak{v}$ vanishes inside the surface layer at time $t_{0}$. Nevertheless, this condition is not strong enough for two reasons. First, if working with the alternative hyperbolicity condition of Definition [3.7, the jet $\mathfrak{v}$ should vanish even in the past of $\underline{t}$. For this reason, we always demand that $\eta_{\underline{t}} \mathfrak{v}=0$, and in case we do not work with the alternative hyperbolicity condition, we simply choose $\underline{t}=t_{\text {min }}$. Second, if working with the hyperbolicity condition of Definition 3.3. we need in addition that the norm $\|\mathfrak{v}\|^{t_{0}}$ vanishes. Moreover, it will be useful to also impose that the symplectic form vanishes in the sense that $\sigma^{t_{0}}(\mathfrak{u}, \mathfrak{v})=0$ for all $\mathfrak{v} \in \mathfrak{J}_{U}$. For convenience, we combine the last two conditions for the surface layer inner product and the symplectic form by expressing them in terms of the surface layer integral $I_{2}^{t_{0}}$ in (3.5). This motivates the definition of the jet space

$$
\underline{\mathfrak{J}}_{t_{0}}:=\left\{\mathfrak{u} \in \mathfrak{J}_{U} \mid \eta_{\underline{t}} \mathfrak{u} \equiv 0 \quad \text { and } \quad I_{2}^{t_{0}}(\mathfrak{u}, \mathfrak{v})=0 \text { for all } \mathfrak{v} \in \mathfrak{J}_{U}\right\}
$$

Similarly, we define the space of jets which vanish at time $t_{\max }$ by

$$
\overline{\mathfrak{J} U}^{t_{\max }}:=\left\{\mathfrak{u} \in \mathfrak{J}_{U} \mid\left(1-\eta_{\bar{t}}\right) \mathfrak{u} \equiv 0 \quad \text { and } \quad I_{2}^{t_{\max }}(\mathfrak{u}, \mathfrak{v})=0 \text { for all } \mathfrak{v} \in \mathfrak{J}_{U}\right\},
$$

where $\bar{t} \in\left[t_{0}, t_{\max }\right]$ is chosen equal to $t_{\max }$ in case we do not work with the alternative hyperbolicity condition.

A strong solution of the Cauchy problem is a jet $\mathfrak{v} \in \mathfrak{J}_{U}$ which satisfies the equations

$$
\Delta \mathfrak{v}=\mathfrak{w} \quad \text { in } L \quad \text { and } \quad \mathfrak{v}-\mathfrak{v}_{0} \in \underline{\mathfrak{J} U}_{t_{0}},
$$

where $\mathfrak{v}_{0} \in \mathfrak{J}_{U}$ is the initial data and $\mathfrak{w}$ is the inhomogeneity. According to (2.18), the inhomogeneity $\mathfrak{w} \in\left(\mathfrak{J}^{\text {test }}\right)^{*}$ is a dual jet. Having the scalar product (3.10) at our disposal, we can identify jets with dual jets. For technical simplicity, we here choose $\mathfrak{w} \in \mathfrak{J}_{U}$.

Proposition 3.12. (uniqueness of strong solutions) If $L$ is a lens-shaped region inside $U$ with foliation $\left(\eta_{t}\right)_{t \in I}$, then the Cauchy problem (3.27) with $\mathfrak{v}_{0}, \mathfrak{w} \in \mathfrak{J}_{U}$ has at most one solution $\mathfrak{v}$ in $L$.

Proof. Let $\mathfrak{v}$ be the difference of two solutions. Then $\mathfrak{v}$ is a solution of the homogeneous equation with zero initial data. Applying Lemma 3.4 or Lemma 3.8, we obtain

$$
\left|\frac{d}{d t}\|\mathfrak{v}\|^{t}\right| \leq c\|\mathfrak{v}\|^{t} \quad \text { and thus } \quad \frac{d}{d t}\left(e^{-c t}\|\mathfrak{v}\|^{t}\right) \leq 0 .
$$

It follows that $\|\mathfrak{v}\|^{t}$ vanishes for all $t$ in the respective interval. Using (3.11), we conclude that $\mathfrak{v}$ vanishes identically in $L$. This gives the result.

3.6. Weak Solutions of the Cauchy Problem. Our goal is to construct solutions of the Cauchy problem (3.27). As usual, replacing $\mathfrak{v}$ by $\mathfrak{v}-\mathfrak{v}_{0}$ and $\mathfrak{w}$ by $\mathfrak{w}-\Delta \mathfrak{v}_{0} \in \mathfrak{J}_{U}$, it suffices to consider the Cauchy problem for zero initial data, i.e.

$$
\Delta \mathfrak{v}=\mathfrak{w} \quad \text { in } U \quad \text { and } \quad \mathfrak{v} \in \underline{\mathfrak{J}}_{t_{0}} .
$$

In order to derive the notion of a weak solution, we take the inner product with a test jet $\mathfrak{u} \in \mathfrak{J}_{U}$ and integrate over space-time. In order to integrate only over $L$, we 
again work with the scalar product $\langle., .\rangle_{L^{2}(L)}$ introduced in (3.13). We thus obtain the equation

$$
\langle\mathfrak{u},(\Delta \mathfrak{v}-\mathfrak{w})\rangle_{L^{2}(L)}=0 \quad \text { for all } \mathfrak{u} \in \mathfrak{J}_{U}
$$

Before going on, we compare this equation with (3.28). If the space $\mathfrak{J}_{U}$ is dense in $L^{2}(L)$, then these equations are equivalent. However, as explained after (2.9), in most situations the space of jets will not be dense. In this case, equation (3.29) contains less information than (3.28). This information loss can be understood similarly as explained after (2.9) by our wish for restricting attention to part of the information contained in the linearized field equations. With this in mind, in what follows we are content with constructing solutions of (3.29).

The following Lemma makes it possible to "integrate by parts."

Lemma 3.13. (Green's formula) For all $\mathfrak{u}, \mathfrak{v} \in \mathfrak{J}_{U}$,

$$
\sigma^{t_{\max }}(\mathfrak{u}, \mathfrak{v})-\sigma^{t_{0}}(\mathfrak{u}, \mathfrak{v})=\langle\mathfrak{u}, \Delta \mathfrak{v}\rangle_{L^{2}(L)}-\langle\Delta \mathfrak{u}, \mathfrak{v}\rangle_{L^{2}(L)} .
$$

Proof. Using the definitions (3.13) and (2.18),

$$
\begin{aligned}
& \langle\mathfrak{u}, \Delta \mathfrak{v}\rangle_{L^{2}(L)}-\langle\Delta \mathfrak{u}, \mathfrak{v}\rangle_{L^{2}(L)}=\int_{U}(\langle\mathfrak{u}, \Delta \mathfrak{v}\rangle-\langle\Delta \mathfrak{u}, \mathfrak{v}\rangle) \eta_{I} d \rho \\
& =\int_{U} d \rho(x) \eta_{I}(x) \nabla_{\mathfrak{u}}\left(\int_{M}\left(\nabla_{1, \mathfrak{v}}+\nabla_{2, \mathfrak{v}}\right) \mathcal{L}(x, y) d \rho(y)-\nabla_{\mathfrak{v}} \mathfrak{s}\right) \\
& \quad-\int_{U} d \rho(x) \eta_{I}(x) \nabla_{\mathfrak{v}}\left(\int_{M}\left(\nabla_{1, \mathfrak{u}}+\nabla_{2, \mathfrak{u}}\right) \mathcal{L}(x, y) d \rho(y)-\nabla_{\mathfrak{u}} \mathfrak{s}\right) .
\end{aligned}
$$

Here the space-time point $x$ is in $L$. Using Definition 3.1 (ii), we get a contribution to the integrals only if $y \in U$. Therefore, we may replace the integration range $M$ by $U$. We thus obtain

$$
\begin{aligned}
& \langle\mathfrak{u}, \Delta \mathfrak{v}\rangle_{L^{2}(L)}-\langle\Delta \mathfrak{u}, \mathfrak{v}\rangle_{L^{2}(L)} \\
& =\int_{U} d \rho(x) \eta_{I}(x) \int_{U} d \rho(y)\left(\nabla_{1, \mathfrak{u}} \nabla_{2, \mathfrak{v}}-\nabla_{2, \mathfrak{u}} \nabla_{1, \mathfrak{v}}\right) \mathcal{L}(x, y) d \rho(y),
\end{aligned}
$$

where we used that, following our convention (2.11), the second derivatives of the Lagrangian are symmetric. Using the definition (3.3) as well as the anti-symmetry of the integrand, the term (3.31) can be rewritten as

$$
\begin{aligned}
& \int_{U} d \rho(x) \eta_{I}(x) \int_{U} d \rho(y)\left(\nabla_{1, \mathfrak{u}} \nabla_{2, \mathfrak{v}}-\nabla_{2, \mathfrak{u}} \nabla_{1, \mathfrak{v}}\right) \mathcal{L}(x, y) d \rho(y) \\
& =\left.\int_{U} d \rho(x) \int_{U} d \rho(y) \eta_{t}(x)\left(\nabla_{1, \mathfrak{u}} \nabla_{2, \mathfrak{v}}-\nabla_{2, \mathfrak{u}} \nabla_{1, \mathfrak{v}}\right) \mathcal{L}(x, y) d \rho(y)\right|_{t_{0}} ^{t_{\max }} \\
& =\left.\int_{U} d \rho(x) \int_{U} d \rho(y)\left(\eta_{t}(x)-\eta_{t}(x) \eta_{t}(y)\right)\left(\nabla_{1, \mathfrak{u}} \nabla_{2, \mathfrak{v}}-\nabla_{2, \mathfrak{u}} \nabla_{1, \mathfrak{v}}\right) \mathcal{L}(x, y) d \rho(y)\right|_{t_{0}} ^{t_{\max }} \\
& =\left.\int_{U} d \rho(x) \int_{U} d \rho(y) \eta_{t}(x)\left(1-\eta_{t}(y)\right)\left(\nabla_{1, \mathfrak{u}} \nabla_{2, \mathfrak{v}}-\nabla_{2, \mathfrak{u}} \nabla_{1, \mathfrak{v}}\right) \mathcal{L}(x, y) d \rho(y)\right|_{t_{0}} ^{t_{\max }} \\
& =\sigma^{t_{\max }}(\mathfrak{u}, \mathfrak{v})-\sigma^{t_{0}}(\mathfrak{u}, \mathfrak{v}) .
\end{aligned}
$$

This gives the result.

Assume that $\mathfrak{v}$ is a strong solution of the Cauchy problem (3.28). Then, applying the above Green's formula, we obtain for any $\mathfrak{u} \in \mathfrak{J}_{U}$,

$$
\langle\mathfrak{u}, \mathfrak{w}\rangle_{L^{2}(L)}=\langle\mathfrak{u}, \Delta \mathfrak{v}\rangle_{L^{2}(L)}=\langle\Delta \mathfrak{u}, \mathfrak{v}\rangle_{L^{2}(L)}-\sigma^{t_{\max }}(\mathfrak{u}, \mathfrak{v})+\sigma^{t_{0}}(\mathfrak{u}, \mathfrak{v}) .
$$


Having implemented the vanishing initial data by the condition $\mathfrak{v} \in \underline{\mathfrak{J}}_{t_{0}}$, the symplectic form vanishes at time $t_{0}$ (note that the symplectic form is obtained by antisymmetrizing the functional $I_{2}$ in (3.25). In order to also get rid of the boundary values at time $t_{\max }$, we restrict attention to test jets which vanish at $t_{\max }$. This leads us to the following definition:

Definition 3.14. A jet $\mathfrak{v} \in L^{2}(L)$ is a weak solution of the Cauchy problem (3.28) if

$$
\langle\Delta \mathfrak{u}, \mathfrak{v}\rangle_{L^{2}(L)}=\langle\mathfrak{u}, \mathfrak{w}\rangle_{L^{2}(L)} \quad \text { for all } \mathfrak{u} \in \overline{\mathfrak{J}} U^{t_{\max }} .
$$

3.7. Existence of Weak Solutions. Our existence proof is inspired by the method invented by K.O. Friedrichs for symmetric hyperbolic systems in [26]; see also [31, Section 5.3] and [22, Chapter 11].

We want to construct a weak solution (3.32). Clearly, the energy estimate of Propositions 3.5 or 3.9 also holds if we exchange the roles of $t_{\max }$ and $t_{0}$, i.e.

$$
\|\mathfrak{u}\|_{L^{2}(L)} \leq \Gamma\|\Delta \mathfrak{u}\|_{L^{2}(L)} \quad \text { for all } \mathfrak{u} \in \overline{\mathfrak{J}} U^{t_{\max }}
$$

(where the constant $\Gamma$ is again given by (3.15)).

We introduce the positive semi-definite bilinear form

$$
\left.<., .>: \overline{\mathfrak{J}}^{t_{\max }} \times{\overline{\mathfrak{J}_{U}}}^{t_{\max }} \rightarrow \mathbb{R}, \quad<\mathfrak{u}, \mathfrak{v}\right\rangle=\langle\Delta \mathfrak{u}, \Delta \mathfrak{v}\rangle_{L^{2}(L)} .
$$

Dividing out the null space and forming the completion, we obtain a Hilbert space $(\mathcal{H},<., .>)$. The corresponding norm is denoted by $\mid\|\cdot\| \|$.

We now consider the linear functional $\langle\mathfrak{w}, .\rangle_{L^{2}(L)}$ on $\overline{\mathfrak{J}} U^{t} t_{\max }$. Applying the Schwarz inequality and (3.33), we obtain

$$
\left|\langle\mathfrak{w}, \mathfrak{u}\rangle_{L^{2}(L)}\right| \leq\|\mathfrak{w}\|_{L^{2}(L)}\|\mathfrak{u}\|_{L^{2}(L)} \leq \Gamma\|\mathfrak{w}\|_{L^{2}(L)}\|\mathfrak{u}\| \|
$$

proving that the linear functional $\langle\mathfrak{w}, .\rangle_{L^{2}(L)}$ on $\overline{\widetilde{J}}_{U}^{t_{\max }}$ is bounded on $\mathcal{H}$. Therefore, it can be extended uniquely to a bounded linear functional on all of $\mathcal{H}$. Moreover, by the Fréchet-Riesz theorem there is a unique vector $V \in \mathcal{H}$ with

$$
\langle\mathfrak{w}, \mathfrak{u}\rangle_{L^{2}(L)}=\langle V, \mathfrak{u}\rangle=\langle\Delta V, \Delta \mathfrak{u}\rangle_{L^{2}(L)} \quad \text { for all } \mathfrak{u} \in \overline{\mathfrak{J} U}^{t_{\max }} .
$$

Hence $\mathfrak{v}:=\Delta V \in L^{2}(L)$ is the desired weak solution. We point out that in the above estimates, the inhomogeneity $\mathfrak{w}$ enters only via its $L^{2}$-norm, making it possible to generalize our methods to $\mathfrak{w} \in L^{2}(L)$. We have obtain the following result:

Theorem 3.15. Assume that $L$ is a lens-shaped region inside $U$ with foliation $\left(\eta_{t}\right)_{t \in I}$ with $I=\left[t_{0}, t_{\max }\right]$. Then for every $\mathfrak{w} \in L^{2}(L)$ there is a weak solution $\mathfrak{v} \in L^{2}(L)$ of the Cauchy problem (3.32). This solution is bounded by

$$
\|\mathfrak{v}\|_{L^{2}(L)} \leq \Gamma\|\mathfrak{w}\|_{L^{2}(L)} .
$$

Proof. It remains to prove the estimate (3.34). To this end, we use that the FréchetRiesz theorem also yields that the norm of $\mathfrak{v}$ equals the sup-norm of the linear functional. Hence

$$
\|\mathfrak{v}\|_{L^{2}(L)}=\|\Delta V\|_{L^{2}(L)}=\|\| v\|\|=\left\|\langle\mathfrak{w}, .\rangle_{L^{2}(L)}\right\|_{\mathcal{H}^{*}} \leq \Gamma\|\mathfrak{w}\|_{L^{2}(L)},
$$

concluding the proof. 
3.8. Are Weak Solutions Unique? We now analyze the uniqueness problem for weak solutions. It is obvious from (3.32) that a weak solution $\mathfrak{v} \in L^{2}(L)$ is unique up to vectors which are orthogonal to all vectors $\Delta \mathfrak{u}$ with $\mathfrak{u} \in \overline{\mathfrak{J} U}^{t_{\text {max }}}$ :

Proposition 3.16. Let $\mathfrak{v}, \tilde{\mathfrak{v}} \in L^{2}(L)$ be two solutions of the weak Cauchy problem (3.32). Then

$$
\mathfrak{v}-\tilde{\mathfrak{v}} \in\left(\Delta\left(\overline{\mathfrak{J}}^{t_{\max }}\right)\right)^{\perp} \subset L^{2}(L) .
$$

As an immediate consequence, we obtain the following result:

Corollary 3.17. If $\Delta\left(\overline{\mathfrak{J}}^{t}{ }^{\max }\right)$ is dense in $L^{2}(L)$, then the weak Cauchy problem (3.32) has a unique solution.

Under the made denseness assumption, this corollary gives an alternative proof of uniqueness of strong solutions (Proposition 3.12). However, this result is only of limited relevance because in most applications, the space $\Delta\left(\widetilde{\mathfrak{J}} U^{t}\right.$ max $)$ will not be dense in $L^{2}(L)$. This corresponds to our general concept explained after (2.9) that by choosing $\mathfrak{J}^{\text {test }}$ we want to restrict attention to the portion of information in the EL equations which is relevant for the application in mind. Using notions from information theory, one can say equivalently that $\mathfrak{J}^{\text {test }}$ determines the bandwidth of the information relevant for our application. With this in mind, the freedom to modify the weak solution according to (3.35) is irrelevant to us because it only affects the information which we disregard. Implementing this point of view mathematically, one could regard the freedom in (3.35) as an equivalence relation and take the uniquely determined equivalence classes as the physically relevant solutions. In order to keep the setting as simple as possible, we here prefer not to form equivalence classes, but to work instead with solutions in $L^{2}(L)$, which are determined only up to the freedom in (3.35). Using this freedom, one can try to find solutions which are particularly simple. For example, the construction of the previous section gives us a canonical solution $\mathfrak{v}=\Delta V$, which is distinguished by the fact that the $L^{2}$-norm of $\mathfrak{v}$ is minimal.

3.9. Weak Solutions in the Future and Past. In the previous section we solved the weak Cauchy problem to the future from the initial time $t_{0}$ to the final time $t_{\max }$. We now analyze how to construct a solution also to the past. Thus we consider a local foliation with $I=\left[t_{\min }, t_{\max }\right]$ of a lens-shaped region $L$ inside $U$. Our goal is to construct a weak solution in $L$ for zero initial data at time $t_{0} \in I$.

In preparation, we reconsider the solution to the future constructed in the previous section. Thus setting

$$
I^{+}=\left[t_{0}, t_{\max }\right] \quad \text { and } \quad L^{+}=\bigcup_{t \in I^{+}} \operatorname{supp} \theta_{t}=\operatorname{supp} \eta_{\left[t_{0}, t_{\max }\right]},
$$

in Theorem 3.15 we constructed a solution $\mathfrak{v} \in L^{2}\left(L^{+}\right)$of the weak equation

$$
\langle\Delta \mathfrak{u}, \mathfrak{v}\rangle_{L^{2}\left(L^{+}\right)}=\langle\mathfrak{u}, \mathfrak{w}\rangle_{L^{2}\left(L^{+}\right)} \quad \text { for all } \mathfrak{u} \in \overline{\mathfrak{J} U}^{t_{\max }} .
$$

We now want to transform this equation with the goal of working instead of the measure $\underline{\rho}_{t_{0}}^{t_{\max }}$ (see (13.13)$)$ with the measure $\rho$. We first note that, by definition of $\overline{\mathfrak{J}} U^{t_{\max }}$ (see $(\underline{3.26})$ ), the jet $\mathfrak{u}$ vanishes identically unless $\eta_{t_{\max }}$ is equal to one. Therefore, the 
right hand side of (3.36) can be rewritten as

$$
\begin{aligned}
\langle\mathfrak{u}, \mathfrak{w}\rangle_{L^{2}\left(L^{+}\right)} & =\int_{L^{+}}\langle\mathfrak{u}(x), \mathfrak{w}(x)\rangle_{x} \eta_{\left[t_{0}, t_{\max }\right]} d \rho \\
& =\int_{L^{+}}\langle\mathfrak{u}(x), \mathfrak{w}(x)\rangle_{x}\left(1-\eta_{t_{0}}\right) d \rho=\langle\mathfrak{u}, \mathfrak{w}\rangle_{L^{2}\left(L^{+},\left(1-\eta_{t_{0}}\right) d \rho\right)} .
\end{aligned}
$$

In order to also remove the dependence of the integration measure on $\eta_{t_{0}}$, we write

$$
\langle\mathfrak{u}, \mathfrak{w}\rangle_{L^{2}\left(L^{+},\left(1-\eta_{t_{0}}\right) d \rho\right)}=\left\langle\mathfrak{u}, \mathfrak{w}^{+}\right\rangle_{L^{2}\left(L^{+}, d \rho\right)}
$$

with

$$
\mathfrak{w}^{+}:=\left(1-\eta_{t_{0}}\right) \mathfrak{w} \in L^{2}\left(L^{+}, d \rho\right) .
$$

On the left hand side of (3.36), we rewrite the integral as

$$
\langle\Delta \mathfrak{u}, \mathfrak{v}\rangle_{L^{2}\left(L^{+}\right)}=\int_{L^{+}}\langle(\Delta \mathfrak{u})(x), \mathfrak{v}(x)\rangle_{x} \eta_{\left[t_{0}, t_{\max }\right]} d \rho=\left\langle\Delta \mathfrak{u}, \mathfrak{v}^{+}\right\rangle_{L^{2}\left(L^{+}, d \rho\right)},
$$

where we set

$$
\mathfrak{v}^{+}:=\eta_{\left[t_{0}, t_{\max }\right]} \mathfrak{v} \in L^{2}\left(L^{+}, d \rho\right)
$$

Thus we can rewrite (3.32) as

$$
\left\langle\Delta \mathfrak{u}, \mathfrak{v}^{+}\right\rangle_{L^{2}\left(L^{+}, d \rho\right)}=\left\langle\mathfrak{u}, \mathfrak{w}^{+}\right\rangle_{L^{2}\left(L^{+}, d \rho\right)} \quad \text { for all } \mathfrak{u} \in \overline{\mathfrak{J} U}^{t_{\max }} .
$$

In this formulation, the existence result of Theorem 3.15] can be stated that for every $\mathfrak{w}^{+}$of the form (3.37) there is a weak solution $\mathfrak{v}^{+} \in L^{2}\left(L^{+}, d \rho\right)$ of (3.39).

Changing the time orientation in an obvious way by reparametrizing $\eta_{t}$ by

$$
\eta_{t} \rightarrow\left(1-\eta_{t^{\prime}}\right) \quad \text { with } \quad t^{\prime}=t_{\max }+t_{\min }-t
$$

and flipping the sign in the hyperbolicity condition 3.11, we obtain similarly a solution $\mathfrak{v}^{-} \in L^{2}\left(L^{-}, d \rho\right)$ to the past, i.e.

$$
\left\langle\Delta \mathfrak{u}, \mathfrak{v}^{-}\right\rangle_{L^{2}\left(L^{-}, d \rho\right)}=\left\langle\mathfrak{u}, \mathfrak{w}^{-}\right\rangle_{L^{2}\left(L^{-}, d \rho\right)} \quad \text { for all } \mathfrak{u} \in \underline{\mathfrak{J}}_{t_{\min }},
$$

where in analogy to (3.37) and (3.38) we now set

$$
\begin{aligned}
\mathfrak{w}^{-} & :=\eta_{t_{0}} \mathfrak{w} \\
\mathfrak{v}^{-} & :=\eta_{\left[t_{\min }, t_{0}\right]} \mathfrak{v} \in L^{2}\left(L^{-}, d \rho\right) .
\end{aligned}
$$

The interesting point is that, according to (3.39), (3.40) and (3.37), (3.41), by extending the solutions $\mathfrak{v}^{+}$and $\mathfrak{v}^{-}$by zero to $L$ and adding them, we get a weak solution in $L$ for the desired inhomogeneity $\mathfrak{w}$. We thus obtain the following result:

Theorem 3.18. Assume that $L$ is a lens-shaped region inside $U$ with foliation $\left(\eta_{t}\right)_{t \in I}$ with $I=\left[t_{\min }, t_{\max }\right]$. Then for every $\mathfrak{w} \in L^{2}(L, d \rho)$ and every $t_{0} \in I$, there is a solution $\hat{\mathfrak{v}} \in L^{2}(L, d \rho)$ of the weak equation

$$
\langle\Delta \mathfrak{u}, \hat{\mathfrak{v}}\rangle_{L^{2}(L, d \rho)}=\langle\mathfrak{u}, \mathfrak{w}\rangle_{L^{2}(L, d \rho)} \quad \text { for all } \mathfrak{u} \in \overline{\widetilde{\mathfrak{J}}}_{t_{\min }}^{t_{\max }},
$$

where $\overline{\mathfrak{J} U}_{t_{\min }}^{t_{\max }}:=\overline{\mathfrak{J}} U^{t_{\max }} \cap \underline{\mathfrak{J}} U_{t_{\min }}$. Moreover, the solution $\hat{\mathfrak{v}}$ vanishes at time $t_{0}$ in the following sense: There is a decomposition

$$
\hat{\mathfrak{v}}=\mathfrak{v}^{+}+\mathfrak{v}^{-} \quad \text { with } \quad \operatorname{supp} \mathfrak{v}^{ \pm} \subset L^{ \pm}
$$

such that $\mathfrak{v}^{+}$and $\mathfrak{v}^{-}$are weak solutions of (3.39) and (3.40), respectively.

The solution $\hat{\mathfrak{v}}$ satisfies the energy estimate

$$
\|\hat{\mathfrak{v}}\|_{L^{2}(L, d \rho)} \leq \Gamma\|\mathfrak{w}\|_{L^{2}(L, d \rho)} \quad \text { with } \quad \Gamma=\sqrt{2} \max \left(\Gamma^{+}, \Gamma^{-}\right),
$$


where $\Gamma^{+}$and $\Gamma^{+}$are the constants in the energy estimate (3.34) for the lens-shaped regions $L^{+}$and $L^{-}$, respectively,

Proof. It remains to prove the energy estimate (3.44). We first consider $\mathfrak{v}^{+}$as given by (3.38). Applying (3.34) to $\mathfrak{v}$ gives

$$
\begin{aligned}
\left\|\mathfrak{v}^{+}\right\|_{L^{2}\left(L^{+}, d \rho\right)}^{2} & =\int_{L^{+}} \eta_{\left[t_{0}, t_{\max }\right]}(x)^{2}\|\mathfrak{v}(x)\|_{x}^{2} d \rho(x) \\
& \leq \int_{L^{+}}\|\mathfrak{v}(x)\|_{x}^{2} \eta_{\left[t_{0}, t_{\max }\right]}(x) d \rho(x) \\
& =\|\mathfrak{v}\|_{L^{2}\left(L^{+}\right)}^{2} \leq\left(\Gamma^{+}\right)^{2}\|\mathfrak{w}\|_{L^{2}\left(L^{+}\right)}^{2} .
\end{aligned}
$$

Adding the corresponding inequality for $\mathfrak{v}^{-}$gives

$$
\begin{aligned}
& \left\|\mathfrak{v}^{+}\right\|_{L^{2}\left(L^{+}, d \rho\right)}^{2}+\left\|\mathfrak{v}^{-}\right\|_{L^{2}\left(L^{-}, d \rho\right)}^{2} \\
& \leq\left(\max \left(\Gamma^{+}, \Gamma^{-}\right)\right)^{2} \int_{L^{+}} \eta_{I}(x)\|\mathfrak{w}(x)\|_{x}^{2} d \rho(x) \\
& \leq\left(\max \left(\Gamma^{+}, \Gamma^{-}\right)\right)^{2}\|\mathfrak{w}(x)\|_{L^{2}(L, d \rho)}^{2} .
\end{aligned}
$$

We finally combine this estimate with the inequality

$$
\|\mathfrak{v}\|_{L^{2}(L, d \rho)}^{2}=\left\|\mathfrak{v}^{+}+\mathfrak{v}^{-}\right\|_{L^{2}(L, d \rho)}^{2} \leq 2\left(\left\|\mathfrak{v}^{+}\right\|_{L^{2}\left(L^{+}, d \rho\right)}^{2}+\left\|\mathfrak{v}^{-}\right\|_{L^{2}\left(L^{-}, d \rho\right)}^{2}\right)
$$

and take the square root.

For clarity, we point out that the energy estimate (3.44) does not hold for $\mathfrak{v}^{+}$separately. Indeed, the norm $\|\mathfrak{w}\|_{L^{2}\left(L^{+}\right)}$in (3.45) cannot be bounded from above by $\left\|\mathfrak{w}^{+}\right\|_{L^{2}\left(L^{+}, d \rho\right)}$, because the inequality

$$
\begin{aligned}
\|\mathfrak{w}\|_{L^{2}\left(L^{+}\right)}^{2} & =\int_{L^{+}}\|\mathfrak{w}(x)\|_{x}^{2} \eta_{\left[t_{0}, t_{\max }\right]}(x) d \rho(x) \\
& \geq \int_{L^{+}} \eta_{\left[t_{0}, t_{\max }\right]}(x)^{2}\|\mathfrak{w}(x)\|_{x}^{2} d \rho(x)=\left\|\mathfrak{w}^{+}\right\|_{L^{2}\left(L^{+}, d \rho\right)}^{2}
\end{aligned}
$$

goes in the wrong direction.

3.10. Restricting and Extending Weak Solutions. We now turn attention to the following questions. Suppose that we are given a weak solution $\mathfrak{v}$ in a lens-shaped region $L$. If $\hat{L}$ is another lens-shaped region contained in $L$, is the restriction of $\mathfrak{v}$ to $\hat{L}$ again a weak solution? Conversely, if $\hat{L}$ is a lens-shaped region containing $L$, can $\mathfrak{v}$ be extended to a weak solution in $\hat{L}$ ?

In preparation, we specify what we mean by "a lens-shaped region is contained in another lens-shaped region." In addition to the obvious inclusion of the lens-shaped regions, we must also impose that the jet spaces and the initial data surface layers fit together.

Definition 3.19. Let $L$ be a lens-shaped region inside $U$ with foliation $\left(\eta_{t}\right)_{t \in\left[t_{\min }, t_{\max }\right]}$, and $\tilde{L}$ a lens-shaped region inside $\tilde{U}$ with foliation $\left(\tilde{\eta}_{t}\right)_{t \in\left[t_{\min }, t_{\max }\right]}$. We say that $L$ is nested in $\tilde{L}$, denoted by

$$
L \prec \tilde{L},
$$

if the following conditions are satisfied:

(i) $L \subset \tilde{L}$ and $U \subset \tilde{U}$ 
(ii) The jet spaces are contained in each other, i.e.

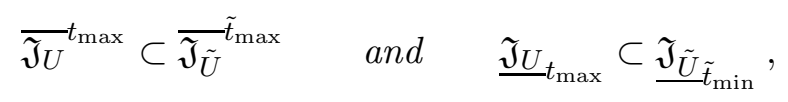

where we extended the jets in $U$ by zero to $\tilde{U}$.

(iii) The initial data surfaces layers are compatible in the sense that for suitable $t_{0} \in$ $\left[t_{\min }, t_{\max }\right]$ and $\tilde{t}_{0} \in\left[\tilde{t}_{\min }, \tilde{t}_{\max }\right]$,

$$
\eta_{t_{0}}=\left.\tilde{\eta}_{\tilde{t}_{0}}\right|_{U}
$$

We begin with the restriction problem. Based on the weak formulation of Theorem 3.18, this problem has a simple answer:

Proposition 3.20. (restriction property) Let $\hat{L} \prec L$ be two nested lens-shaped regions. Moreover, let $\mathfrak{v} \in L^{2}(L, d \rho)$ be the weak solution of the Cauchy problem for the inhomogeneity $\mathfrak{w} \in L^{2}(L, d \rho)$ with zero initial data at time $t_{0}$ as constructed in Theorem 3.18. Then the jet $\hat{\mathfrak{v}}:=\left.\mathfrak{v}\right|_{\hat{L}} \in L^{2}(\hat{L}, d \rho)$ is a weak solution for the inhomogeneity $\mathfrak{w}$ with zero initial data at time $\hat{t}_{0}$.

Proof. The result follows immediately from the fact that the weak equations (3.39), (3.40) and (3.43) remain valid if the jet space used for testing is made smaller.

The extension problem is more subtle. The basic difficulty can be understood as follows. Suppose that $\mathfrak{v}$ and $\hat{\mathfrak{v}}$ are weak solutions in $L$ respectively $\hat{L}$ with $L \prec \hat{L}$. Due to the nonlocality of the operator $\Delta$, we cannot expect that $\left.\hat{\mathfrak{v}}\right|_{L}=\mathfrak{v}$ (due to the restriction property of Proposition [3.20, we know that $\left.\hat{\mathfrak{v}}\right|_{L}$ is again a weak solution in $L$, but in view of the non-uniqueness result of Proposition 3.16 this does not imply that $\left.\hat{\mathfrak{v}}\right|_{L}=\mathfrak{v}$ ). For example for constructing global solution (for details see Section 4.3 below), it is important to quantify $\left.\hat{\mathfrak{v}}^{+}\right|_{L}-\mathfrak{v}^{+}$depending on the size of the lens-shaped region $\hat{L}$. Since the necessary estimates are a bit technical, we begin with the following simpler question: Is there an open set $\Omega \subset L$ in which $\hat{\mathfrak{v}}$ coincides with $\mathfrak{v}$ ? The next proposition shows that, under certain conditions, this question has an affirmative answer. We first state and prove our result and explain it afterward.

Definition 3.21. Let $A, B \subset L^{2}(M, d \rho)$ be two (not necessarily closed) subspaces of the Hilbert space of square-integrable jets. Moreover let $V \subset M$ be a subset of spacetime. The subspace $A$ shields $V$ from $B$ if all jets in $A^{\perp} \cap \bar{B}$ vanish identically in $V$.

We introduce the jet spaces 1

$$
\begin{gathered}
\mathfrak{K}\left(L^{+}\right):=\operatorname{span}\left\{\eta_{\left[t, t_{\text {max }}\right]} \Delta\left(\overline{\mathfrak{J}} U_{t_{\text {max }}}\right) \mid t \in\left[t_{0}, t_{\text {max }}\right]\right\} \\
\mathfrak{K}\left(L^{-}\right):=\operatorname{span}\left\{\eta_{\left[t_{\text {min }}, t\right]} \Delta\left(\underline{\mathfrak{J}}_{t_{\text {min }}}\right) \mid t \in\left[t_{\text {min }}, t_{0}\right]\right\}
\end{gathered}
$$

(and similarly with hats), where the multiplication of a function in space-time with a jet space means that all jets are multiplied pointwise by this function. We extend all jets by zero to all of $M$ and consider them as vectors in $L^{2}(M, d \rho)$.

\footnotetext{
${ }^{1}$ At this stage, it would be sufficient to define the set $\mathfrak{K}\left(L^{+}\right)$(and similarly $\mathfrak{K}\left(L^{-}\right)$) by $\mathfrak{K}\left(L^{+}\right)=$ $\eta_{\left[t_{0}, t_{\max }\right]} \Delta\left(\overline{\mathfrak{J}} U^{t_{\max }}\right)$. The more general definition with $t \in\left[t_{0}, t_{\max }\right]$ is of advantage in view of the constructions in Section 4.4
} 
Proposition 3.22. (extension property) Let $L \prec \hat{L}$ be two nested lens-shaped regions. Moreover, let $\mathfrak{v} \in L^{2}(L, d \rho)$ be the weak solution of the Cauchy problem with inhomogeneity $\mathfrak{w}:=\left.\hat{\mathfrak{w}}\right|_{U}$ with zero initial data at time $t_{0}$ as constructed in Theorem 3.18. Finally, assume that $\Omega \subset L$ is an open set such that

$$
\begin{array}{lll}
\chi_{L^{+}} \Delta\left(\overline{\mathfrak{J}}_{U} t_{\text {max }}\right) & \text { shields } \Omega \text { from } & \operatorname{span}\left(\mathfrak{K}\left(\hat{L}^{+}\right), \mathfrak{K}\left(L^{+}\right)\right) \\
\chi_{L^{-}} \Delta\left(\underline{\mathfrak{J}}_{t_{\text {min }}}\right) & \text { shields } \Omega \text { from } & \operatorname{span}\left(\mathfrak{K}\left(\hat{L}^{-}\right), \mathfrak{K}\left(L^{-}\right)\right)
\end{array}
$$

(where $\chi_{L^{ \pm}}$denote the characteristic functions of $L^{ \pm}$). Then the solution $\hat{\mathfrak{v}}$ of the Cauchy problem in $\hat{L}$ with zero initial data at time $\hat{t}_{0}$ constructed in Theorem 3.18 has the property that it extends $\mathfrak{v}$ in $\Omega$ in the sense that

$$
\left.\hat{\mathfrak{v}}\right|_{\Omega}=\left.\mathfrak{v}\right|_{\Omega} .
$$

Proof. We let $\mathfrak{v}$ and $\hat{\mathfrak{v}}$ be the solutions constructed in Theorem 3.18, It suffices to consider the solutions $\mathfrak{v}^{+}$and $\hat{\mathfrak{v}}^{+}$in the future (as defined by (3.39)), because the solutions to the past are treated analogously.

The first step is to show that

$$
\mathfrak{v}^{+} \in \overline{\mathfrak{K}\left(L^{+}\right)} \quad \text { and } \quad \hat{\mathfrak{v}}^{+} \in \overline{\mathfrak{K}\left(\hat{L}^{+}\right)}
$$

(where the overline denotes the closure in $L^{2}(M, d \rho)$ ). To this end, we note that the solution constructed in Theorem 3.15 lies in the $L^{2}\left(L^{+}\right)$-completion of $\Delta \overline{\mathfrak{J}} U^{t_{\max }}$. The solution $\hat{\mathfrak{v}}^{+}$is obtained from this solution by multiplication with cutoff functions (3.38). We also saw that this solution is in $L^{2}(L, d \rho)$. Hence it lies in the $L^{2}$-completion of $\mathfrak{K}\left(L^{+}\right)$as defined in (3.46). The argument for $\mathfrak{K}\left(\hat{L}^{+}\right)$is the same.

According to (3.39), $\mathfrak{v}^{+}$and $\hat{\mathfrak{v}}^{+}$satisfy the weak equations

$$
\begin{array}{ll}
\left\langle\Delta \mathfrak{u}, \mathfrak{v}^{+}\right\rangle_{L^{2}\left(L^{+}, d \rho\right)}=\left\langle\mathfrak{u}, \mathfrak{w}^{+}\right\rangle_{L^{2}\left(L^{+}, d \rho\right)} & \text { for all } \mathfrak{u} \in \overline{\mathfrak{J}} U^{t_{\max }} \\
\left\langle\Delta \mathfrak{u}, \hat{\mathfrak{v}}^{+}\right\rangle_{L^{2}\left(\hat{L}^{+}, d \rho\right)}=\left\langle\mathfrak{u}, \hat{\mathfrak{w}}^{+}\right\rangle_{L^{2}\left(\hat{L}^{+}, d \rho\right)} & \text { for all } \mathfrak{u} \in \overline{\mathfrak{J}}_{\hat{U}}^{\hat{t}_{\max }} .
\end{array}
$$

According to Definition 3.19 (ii), we may restrict the second equation to $\mathfrak{u} \in \overline{\widetilde{J}} U^{t_{\max }}$ and combine it with the first equation to obtain

$$
\left\langle\Delta \mathfrak{u}, \hat{\mathfrak{v}}^{+}-\mathfrak{v}^{+}\right\rangle_{L^{2}\left(L^{+}, d \rho\right)}=0 \quad \text { for all } \mathfrak{u} \in{\overline{\mathfrak{J}_{U}}}^{t_{\max }} .
$$

In other words, extending $\hat{\mathfrak{v}}^{+}-\mathfrak{v}^{+}$by zero to all of $M$, this function lies in the orthogonal complement of $\chi_{L^{+}} \Delta\left(\overline{\mathfrak{J} U}^{t} t_{\max }\right)$. Moreover, from (3.48) we know that the extension of $\hat{\mathfrak{v}}^{+}-\mathfrak{v}^{+}$lies in the completion of the span of $\mathfrak{K}\left(\hat{L}^{+}\right)$and $\mathfrak{K}\left(L^{+}\right)$. The shielding property implies that $\hat{\mathfrak{v}}^{+}-\mathfrak{v}^{+}$vanishes identically in $\Omega$. This concludes the proof.

We now explain the concept of shielding and discuss if the conditions (3.47) are sensible assumptions for the applications in mind. Intuitively speaking, the shielding property of Definition 3.21 means that, restricting attention to the space-time region $V$, the jets are described completely by the jets in $A$. This intuitive picture is made precise by demanding that all jets in the orthogonal complement of $A$ should vanish identically in $V$. In order to illustrate the notion of shielding, we now discuss a few examples, for simplicity for real-valued functions on the real line. In the first example, we choose the Hilbert space $L^{2}(\mathbb{R})$ and the subspaces

$$
A:=L^{2}((0,1)) \subset B:=L^{2}(\mathbb{R}) .
$$



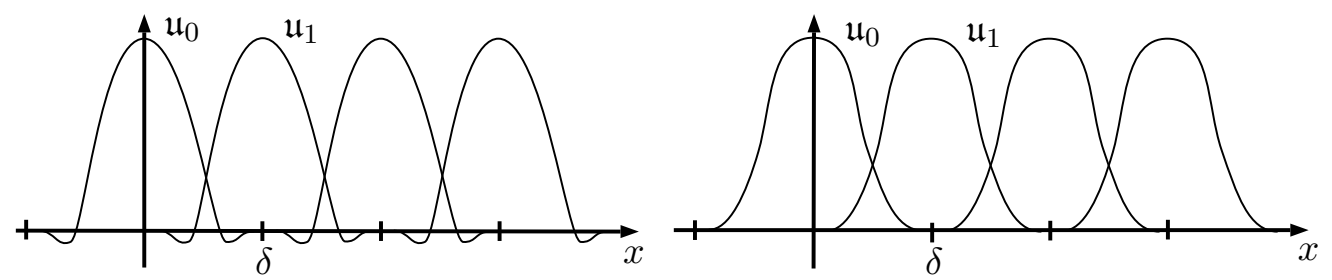

FiguRE 2. Example with shielding (left) and without shielding (right).

Moreover, we choose $V=(0,1)$. In this example, the functions in the space

$$
A^{\perp} \cap \bar{B}=L^{2}(\mathbb{R} \backslash(0,1))
$$

vanish identically in $V$. Thus $A$ shields $V$ from $B$. The situation is similar if we consider smooth functions, like in the example

$$
A:=\left\{\mathfrak{u} \in C_{0}^{\infty}(\mathbb{R}) \mid \operatorname{supp} \mathfrak{u} \subset[0,1]\right\} \subset B:=C_{0}^{\infty}(\mathbb{R}) .
$$

In this example, the set $A^{\perp} \cap \bar{B}$ is again given by (3.49), showing that $A$ again shields $V$ in $B$.

As explained after (2.9), the purpose of $\mathfrak{J}^{\text {test }}$ is to restrict attention to part of the information contained in the EL equations. Of particular interest are situations when the jets describe only the macroscopic behavior but disregards structures which are smaller than a microscopic length scale $\delta$. In order to illustrate this situation in a simple example, we consider the functions

$$
\mathfrak{u}_{\ell}(x):=\eta(x-\delta \ell), \quad \ell \in \mathbb{N}_{0},
$$

where $\eta \in C_{0}^{\infty}((-\delta, \delta))$ (see Figure 2). We consider again the Hilbert space $L^{2}(\mathbb{R})$ and choose

$$
A:=\operatorname{span}\left(\mathfrak{u}_{0}, \ldots, \mathfrak{u}_{L}\right) \subset B:=\operatorname{span}\left(\mathfrak{u}_{0}, \mathfrak{u}_{1}, \ldots\right),
$$

with a parameter $L>2$. Moreover, we choose $V=[0, \delta]$. In this example, the shielding property is more subtle. If the vectors $\mathfrak{u}_{\ell}$ are orthogonal; i.e. by symmetry if

$$
0=\left\langle\mathfrak{u}_{0}, \mathfrak{u}_{1}\right\rangle_{L^{2}(\mathbb{R})}=\int_{0}^{\delta} \eta(x) \eta(\delta-x) d x
$$

(see the left of Figure 2), then

$$
A^{\perp} \cap \bar{B}=\overline{\operatorname{span}\left(\mathfrak{u}_{L+1}, \mathfrak{u}_{L+2}, \ldots\right)},
$$

showing that $A$ again shields $V$ from $B$. However, if the vectors $\eta_{\ell}$ are not orthogonal, then the space $A^{\perp} \cap \bar{B}$ contains functions which do not vanish identically in $V$. Thus the shielding property is violated, although, intuitively speaking, $A$ does describe the jets in $V$ completely. The reason for this seeming inconsistency is that taking the orthogonal complement also involves the behavior of the functions in $A$ outside $V$. More precisely, a short computation shows that the space $A^{\perp} \cap \bar{B}$ is spanned by the vector

$$
\mathfrak{u}_{L+1}-\kappa \mathfrak{u}_{L}+\kappa^{2} \mathfrak{u}_{L-1}-\cdots+(-\kappa)^{L+1} \mathfrak{u}_{0} \quad \text { with } \quad \kappa:=\frac{\left\langle\mathfrak{u}_{0}, \mathfrak{u}_{1}\right\rangle_{L^{2}(\mathbb{R})}}{\left\|u_{0}\right\|_{L^{2}(\mathbb{R})}^{2}}
$$

as well as the vectors $\mathfrak{u}_{L+2}, \mathfrak{u}_{L+3}, \ldots$. The overlap of the jets $\mathfrak{u}_{L} \in A$ with $\mathfrak{u}_{L+1} \notin A$ has the effect that the vector (3.50) does not vanish identically in $V$. But at least, 
equation (3.50) shows that the error of shielding decays exponentially if $L$ is increased, in the sense that for all functions in $A^{\perp} \cap \bar{B}$ the inequality

$$
\|\mathfrak{u}\|_{L^{2}(V)} \leq e^{-\kappa L}\|\mathfrak{u}\|_{L^{2}(\mathbb{R})}
$$

holds.

We next consider the typical length scales. As mentioned above, $\delta$ is a microscopic length scale (which can be thought of as the Planck scale). Therefore, the inequality (3.51) shows that shielding takes place on a microscopic length scale. Taking into account that all other length scales (like for example the constant $1 / c$ in the hyperbolic estimate (3.23) and (3.15) ) are macroscopic, from the physical point of view the shielding assumption (3.47) is an extremely good approximation.

From the mathematical perspective, however, the assumption (3.47) is too strong because it is violated in most applications of interest. Inspecting how (3.47) enters the proof of Proposition 3.22, one also sees that the extension property does not hold. Instead, extending a solution necessarily changes the solution slightly in $V$. While this effect is not surprising in view of the nonlocality of the causal action principle, it is a major complication of the mathematical analysis. For a mathematically convincing treatment, in (3.47) we must allow for an error term of a form similar to (3.51), and this error term must be controlled in the subsequent estimates. This method will be introduced when constructing global solutions in Section 4.3 .

3.11. Estimates of the Initial Data. We now analyze if a weak solution is a strong solution. It is most convenient to work with the weak formulation of Theorem 3.18 , Thus let $\mathfrak{v} \in L^{2}(L, d \rho)$ be a weak solution of (3.43) with zero initial data at time $t_{0}$ (as is made precise in the statement of Theorem (3.18). At this point, it is convenient to work instead of $\rho$ with the measure $\underline{\rho}_{t_{\min }}^{t_{\max }}$. To this end, we divide the solution in (3.43) by the function $\eta_{I}$ (which is possible in view of (3.38) and (3.42)). On the right side of (3.43) we may change the integration measure because the jet $\mathfrak{u}$ vanishes unless $\eta_{I}$ is identically equal to one; see (3.25) and (3.26)). We thus obtain a solution of the weak equation

$$
\langle\Delta \mathfrak{u}, \mathfrak{v}\rangle_{L^{2}(L)}=\langle\mathfrak{u}, \mathfrak{w}\rangle_{L^{2}(L)} \quad \text { for all } \mathfrak{u} \in \overline{\mathfrak{J} U}_{t_{\min }}^{t_{\max }},
$$

where we used the abbreviation $L^{2}(L)=L^{2}\left(L, d \bar{\rho}_{t_{\min }}^{t_{\max }}\right)$. After extending $\mathfrak{v}$ by zero to $U$, we can apply the transformations of the integrals in the proof of Lemma 3.13 and exploit that the symplectic form in (3.30) vanishes in view of the definition of the jet space $\overline{\mathfrak{J} U}_{t}^{t_{\max }}$ (see (3.25) and $(\underline{3.26})$ ). We conclude that $\langle\mathfrak{u}, \Delta \mathfrak{v}\rangle_{L^{2}(L)}$ is well-defined and satisfies the Green's formula (3.30) with a vanishing left side. As a consequence, the above weak equation can be written as as

$$
\langle\mathfrak{u},(\Delta \mathfrak{v}-\mathfrak{w})\rangle_{L^{2}(L)}=0 \quad \text { for all } \mathfrak{u} \in{\overline{\mathfrak{J}_{U}}}_{t_{\min }}
$$

This is the strong equation tested with the jet $\mathfrak{u}$. Similar as explained after (3.29), this is precisely the equation we are aiming for.

The remaining question is whether and in which sense the weak solution satisfies the initial conditions. Recall that for the strong solution in (3.28), the trivial initial data was imposed by demanding that $\mathfrak{v} \in \underline{\mathfrak{J}}_{t_{0}}$. In the weak formulation (3.32), however, the initial condition is encoded implicitly by the fact that the test jets $\mathfrak{u} \in \overline{\mathfrak{J}} U^{t_{\max }}$ do not need to vanish at time $t_{0}$. But does this equation imply that $\mathfrak{v}$ vanishes at time $t_{0}$ ? If yes, in which sense? These questions are rather subtle. In order to understand the 
basic difficulty, we "integrate by parts" in (3.32) with the help of the Green's formula in Lemma 3.13. This gives the equation

$$
\langle\mathfrak{u},(\Delta \mathfrak{v}-\mathfrak{w})\rangle_{L^{2}(L)}=\sigma^{t_{0}}\left(\mathfrak{u}, \mathfrak{v}-\mathfrak{v}_{0}\right) \quad \text { for all } \mathfrak{u} \in \overline{\mathfrak{J}}^{t_{\max }} .
$$

Similar to (3.52), the left side of this equation is the strong equation tested with $\mathfrak{u}$. However, this is the formulation where we solve only to the future, making it impossible to deduce from (3.52) that the left side of (3.53) vanishes. As a consequence, we cannot conclude that the right side of (3.53) is zero. In other words, (3.53) involves a combination of volume and boundary terms, making it impossible to read off the boundary data. The situation does not become easier in Section 3.9 when constructing solutions to the future and past, because in the weak formulation of Theorem 3.18, the boundary conditions are encoded only implicitly in the weak equations (3.39) and (3.40).

In order to clarify the situation, we now give a method for estimating the initial data $\|\mathfrak{v}\|^{t_{0}}$. We again use the concept of shielding (see Definition 3.21). The assumption of shielding should be regarded mainly as a technical simplification. Indeed, in situations where shielding does not hold (as explained at the end of Section 3.10), the following method can still be used if combined in a straightforward way with quantitative estimates of the error of shielding (see Definition 4.8 and the proofs of Theorem 4.9 in Section 4.3 below).

Let $\mathfrak{v}$ be the weak solution constructed in Theorem 3.18. Moreover, choosing a subinterval $\hat{I}:=\left[\hat{t}_{\min }, \hat{t}_{\max }\right] \subset I$ and setting

$$
\hat{L}:=\bigcup_{t \in \hat{I}} \operatorname{supp} \theta_{t}
$$

the set $\hat{L}$ is again a lens-shaped region inside $U$, having the local foliation $\left(\eta_{t}\right)_{t \in \hat{I}}$. We again define the sets $\mathfrak{K}\left(L^{ \pm}\right)$by (3.46) (and similarly with hats).

Theorem 3.23. Assume that

$$
\begin{array}{lll}
\chi_{\hat{L}^{+}} \Delta\left(\overline{\mathfrak{J}}_{U} \hat{t}_{\text {max }}\right) & \text { shields } \operatorname{supp} \theta_{t_{0}} \text { from } & \operatorname{span}\left(\mathfrak{K}\left(\hat{L}^{+}\right), \mathfrak{K}\left(L^{+}\right)\right) \\
\chi_{\hat{L}^{-}} \Delta\left(\underline{\mathfrak{J}}_{U} \hat{t}_{\text {min }}\right) & \text { shields } \operatorname{supp} \theta_{t_{0}} \text { from } & \operatorname{span}\left(\mathfrak{K}\left(\hat{L}^{-}\right), \mathfrak{K}\left(L^{-}\right)\right) .
\end{array}
$$

Then

where the constant $\hat{c}$ is given by

$$
\|\mathfrak{v}\|_{L^{2}\left(L, d \rho_{t_{0}}\right)} \leq \hat{c}\|\mathfrak{w}\|_{L^{2}(\hat{L}, d \rho)}
$$

$$
\hat{c}=4 C e^{2 c\left(\hat{t}_{\max }-\hat{t}_{\min }\right)}\left(\hat{t}_{\max }-\hat{t}_{\min }\right) \sqrt{\left\|\theta_{t_{0}}\right\|_{L^{\infty}}} .
$$

Proof. We first consider the solution $\mathfrak{v}^{+}$in $L^{+}$and the corresponding solution $\hat{\mathfrak{v}}^{+}$ in $\hat{L}^{+}$. Applying Proposition 3.22, these solutions coincide in $\operatorname{supp} \theta_{t_{0}}$. Similarly, the solutions $\mathfrak{v}^{-}$and $\hat{\mathfrak{v}}^{-}$coincide on $\operatorname{supp} \theta_{t_{0}}$. As a consequence, the functions $\mathfrak{v}$ and $\hat{\mathfrak{v}}$ coincide on $\operatorname{supp} \theta_{t_{0}}$. Therefore, it suffices to estimate $\hat{\mathfrak{v}}$.

The energy estimate (3.44) gives

$$
\|\hat{\mathfrak{v}}\|_{L^{2}(L, d \rho)} \leq \hat{\Gamma}\|\mathfrak{w}\|_{L^{2}(L, d \rho)},
$$

where we choose $\hat{\Gamma}=2\left(\Gamma^{+}+\Gamma^{-}\right)$with $\Gamma^{+}$and $\Gamma^{-}$according to (3.15). Finally, we estimate the norm on the left by

$$
\|\hat{\mathfrak{v}}\|_{L^{2}(L, d \rho)}^{2}=\int_{L}\|\hat{\mathfrak{v}}(x)\|_{x}^{2} d \rho(x) \geq \frac{1}{\left\|\theta_{t_{0}}\right\|_{L^{\infty}}} \int_{L} \theta_{t_{0}}(x)\|\hat{\mathfrak{v}}(x)\|_{x}^{2} d \rho(x) .
$$


This gives the result.

We now explain this result and formulate two corollaries. The main point of the above estimate is that the constant $\hat{c}$ in (3.55) becomes small if $\hat{t}_{\max }-\hat{t}_{\min }$ tend to zero. This means that the error of the initial values is directly related to the shielding. In physical applications, shielding occurs on a microscopic scale $\delta$ (as explained at the end of Section 3.10). Therefore, we can choose $\hat{t}_{\max }-\hat{t}_{\min } \sim \delta$, showing that the error in the initial data is extremely small. Moreover, one sees that an error in the initial data occurs only if $\mathfrak{w}$ does not vanish near the boundary, as is made precise by the following statement.

Corollary 3.24. Under the assumptions of Theorem 3.23, the following implication holds:

$$
\left.\left.\mathfrak{w}\right|_{\hat{L}} \equiv 0 \quad \Longrightarrow \quad \mathfrak{v}\right|_{\hat{L}} \equiv 0 \text {. }
$$

We finally rewrite the above results for solutions of the Cauchy problem with nontrivial initial data. To this end, we return to the strong Cauchy problem for non-trivial initial data in (3.27). The method for solving this equation is to construct a strong solution $\tilde{\mathfrak{v}}$ for the inhomogeneity $\tilde{\mathfrak{w}}=\mathfrak{w}-\Delta \mathfrak{v}_{0}$ with trivial initial data (3.27) and to set $\mathfrak{v}=\tilde{\mathfrak{v}}+\mathfrak{v}_{0}$. Now suppose that $\tilde{\mathfrak{v}} \in L^{2}(L)$ is a corresponding weak solution as constructed in Theorem 3.18. Then the jet $\mathfrak{v}:=\tilde{\mathfrak{v}}+\mathfrak{v}_{0}$ satisfies in generalization of (3.43) the weak equation

$$
\left\langle\Delta \mathfrak{u},\left(\mathfrak{v}-\mathfrak{v}_{0}\right)\right\rangle_{L^{2}(L, d \rho)}=\left\langle\mathfrak{u},\left(\mathfrak{w}-\Delta \mathfrak{v}_{0}\right)\right\rangle_{L^{2}(L, d \rho)} \quad \text { for all } \mathfrak{u} \in \overline{\mathfrak{J}} U_{t_{\min }}^{t_{\max }} .
$$

The equations for $\mathfrak{v}^{ \pm}$as well as the estimate of Theorem 3.23 are obtained similarly by the simple replacements

$$
\mathfrak{v} \rightarrow \mathfrak{v}-\mathfrak{v}_{0} \quad \text { and } \quad \mathfrak{w} \rightarrow \mathfrak{w}-\Delta \mathfrak{v}_{0} .
$$

This gives the following result:

Corollary 3.25. Assume that the shielding property (3.54) holds. Then

$$
\left\|\mathfrak{v}-\mathfrak{v}_{0}\right\|_{L^{2}\left(\hat{L}, d \rho t_{0}\right)} \leq \hat{c}\left\|\Delta \mathfrak{v}_{0}-\mathfrak{w}\right\|_{L^{2}(\hat{L}, d \rho)}
$$

with $\hat{c}$ as in (3.55). Moreover, the following implication holds:

$$
\left.\left.\left(\Delta \mathfrak{v}_{0}-\mathfrak{w}\right)\right|_{\hat{L}} \equiv 0 \quad \Longrightarrow \quad\left(\mathfrak{v}-\mathfrak{v}_{0}\right)\right|_{\hat{L}} \equiv 0 .
$$

Intuitively speaking, this result can be understood as follows: If the Cauchy problem for the initial value $\mathfrak{v}_{0}$ can be solved "locally" in a small lens-shaped region $\hat{L}$, then it also has a solution in the larger lens-shaped region $L$. The size of $\hat{L}$ is determined by the shielding of the jets; in physical applications this size will be of the order of the microscopic length scale $\delta$. This result fits nicely to our earlier concept of prescribing the initial data not on a hypersurface, but in a surface layer. In applications, it seems natural and easiest to choose the width of the surface layers of the same order as the length scale $\delta$ of shielding.

\section{Causal Structure and Global Hyperbolicity}

4.1. Causal Cones and Transitive Causal Relations. In this section we shall clarify the causal structure of space-time by introducing causal cones. In particular, we shall get the connection to partially ordered sets. Our method is to construct cone structures from the lens-shaped regions. The construction is based on the assumption 


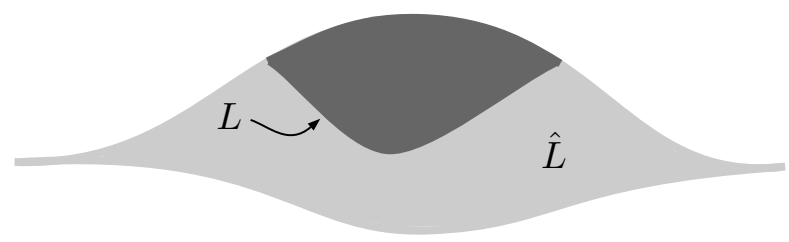

FiguRE 3. Two lens-shaped regions $L$ and $\hat{L}$ with $L \ll \hat{L}$.

that there are arbitrarily large lens-shaped regions, as is made precise by the following notion of compact hyperbolicity:

Definition 4.1. Space-time is locally hyperbolic if every $x \in M$ has an open neighborhood $\Omega$ contained in a lens-shaped region $L$. It is compactly hyperbolic if every compact subset $K \subset M$ has an open neighborhood $\Omega \supset K$ contained in a lens-shaped region $L$.

In what follows, for every lens-shaped region we always choose a corresponding local foliation $\left(\eta_{t}\right)_{t \in\left[0, t_{\max }\right]}$ inside a set $U$ (see Definition [3.11). For ease in notation, the corresponding objects $\left(L, U, \eta_{t}\right)$ always carry the same indices, tildes and hats.

Since $M$ is $\sigma$-compact (see the last paragraph of Section 2.1 on page 6), we can choose an exhaustion of $M$ by compact sets $\left(K_{n}\right)_{n \in \mathbb{N}}$, i.e.

$$
K_{1} \subset \stackrel{\circ}{K_{2}} \subset K_{2} \subset \stackrel{\circ}{K}_{3} \subset \cdots \quad \text { and } \bigcup_{n \in \mathbb{N}} K_{n}=M .
$$

In the following constructions, we will frequently work with such exhaustions. Clearly, we must always verify that the resulting objects and notions do not depend on the choice of the exhaustion.

Definition 4.2. A lens-shaped region $L$ is past-contained in $\hat{L}$, denoted by $L \ll \hat{L}$, if

$$
U=\hat{U} \quad \text { and } \quad \overline{\mathfrak{J}}^{t_{\max }} \supset{\overline{\mathfrak{J}_{\hat{U}}}}^{t_{\max }} .
$$

The inclusion in (4.1) means that $L$ involves weaker boundary conditions at $t_{\max }$ than $\hat{L}$, which in turn can be understood intuitively by the condition that the future boundary of $L$ must be contained in the future boundary of $\hat{L}$ (see Figure 3).

Definition 4.3. Let $\left(K_{n}\right)_{n \in \mathbb{N}}$ be an exhaustion of $M$ by compact sets. Given $x \in M$ and $N \in \mathbb{N}$, the set $J_{N}^{\vee}(x) \subset M$ is defined as the set of all space-time points $y$ with the property that for all lens-shaped regions $\hat{L}$ and $L$ with $L \ll \hat{L} \supset K_{N}$ and $x$ contained in the interior of $L$, the point $y$ lies in $L$, i.e.

$$
J_{N}^{\vee}(x):=\left\{y \in M \mid \underset{\hat{L} \supset K_{N}}{\forall} \underset{L \ll \hat{L}}{\forall}: x \in \stackrel{\circ}{\forall} \Longrightarrow y \in L\right\} .
$$

Its interior is denoted by $I_{N}^{\vee}(x)$,

$$
I_{N}^{\vee}(x):=\overbrace{J_{N}^{\vee}(x)}^{\circ} .
$$

Moreover, the sets $J^{\vee}(x)$ and $I^{\vee}(x)$ are defined by taking the union over $N$,

$$
J^{\vee}(x):=\bigcup_{N \in \mathbb{N}} J_{N}^{\vee}(x) \quad \text { and } \quad I^{\vee}(x):=\bigcup_{N \in \mathbb{N}} I_{N}^{\vee}(x) .
$$


Finally, for a compact set $K \subset M$, we set

$$
J^{\vee}(K):=\bigcup_{x \in K} J^{\vee}(x) \quad \text { and } \quad I^{\vee}(K):=\bigcup_{x \in K} I^{\vee}(x) .
$$

We refer to $J^{\vee}$ as the future cone and $I^{\vee}$ as the open future cone.

We note that, being the union of open sets, the open future light cone is indeed an open subset of $M$. We next verify that the definitions are independent of the choice of the exhaustion:

Proposition 4.4. The sets $J^{\vee}(x)$ and $I^{\vee}(x)$ do not depend on the choice of the exhaustion $\left(K_{n}\right)_{n \in \mathbb{N}}$.

Proof. Using quantifiers, the sets $J^{\vee}(x)$ and $I^{\vee}(x)$ can be written as

$$
\begin{aligned}
& J^{\vee}(x)=\left\{y \in M \mid \underset{N \in \mathbb{N}}{\exists} \underset{\hat{L} \supset K_{N}}{\forall} \underset{L \ll \hat{L}}{\forall}: x \in \stackrel{\circ}{\forall} \Longrightarrow y \in L\right\} \\
& I^{\vee}(x)=\left\{y \in M \mid \underset{N \in \mathbb{N}}{\exists} \underset{U_{y} \ni y}{\exists} \underset{\hat{L} \supset K_{N}}{\forall} \underset{L \ll \hat{L}}{\forall}: x \in \stackrel{\circ}{\forall} \Longrightarrow U_{y} \subset L\right\},
\end{aligned}
$$

where $U_{y}$ denotes an open neighborhood of $y$ in $M$. Since the sets $J_{N}^{\vee}(x)$ are increasing, i.e.

$$
J_{1}^{\vee}(x) \subset J_{2}^{\vee}(x) \subset \cdots,
$$

their union $J^{\vee}(x)$ is characterized purely by the lens-shaped regions enclosing large compact sets $K_{N}$ for large $N$. In particular, it is independent of the choice of the exhaustion. The proof for $I^{\vee}(x)$ is similar.

We also point out that the set $I^{\vee}(x)$ in general does not coincide with the interior of $J^{\vee}(x)$. Namely, writing this interior as

$$
\overbrace{J^{\vee}(x)}^{\circ}=\left\{y \in M \mid \underset{U_{y} \ni y}{\exists} \underset{\tilde{y} \in U_{y}}{\forall} \underset{N \in \mathbb{N}}{\exists} \underset{\hat{L} \supset K_{N}}{\forall} \underset{L \ll \hat{L}}{\forall}: x \in \stackrel{\circ}{\forall} \Longrightarrow \tilde{y} \in L\right\},
$$

the parameter $N$ may depend on $\tilde{y}$, giving rise to a weaker condition. Therefore, in general we only have the inclusion

$$
I^{\vee}(x) \subset \overbrace{J^{\vee}(x)}^{\circ}
$$

This situation resembles similar results in low regularity Lorentzian geometry; see for example [7, 28]. Before coming back to this subtle point (see Section 4.5), we prove that the sets $I^{\vee}(x)$ induce a transitive causal relation on space-time:

Theorem 4.5. The partial relation defined by the sets $I^{\vee}(x)$ is transitive, meaning that

$$
y \in I^{\vee}(x) \quad \text { and } \quad z \in I^{\vee}(y) \quad \Longrightarrow \quad z \in I^{\vee}(x) .
$$

Proof. Let $\left(K_{n}\right)_{n \in \mathbb{N}}$ be an exhaustion by compact sets. Then, by our definition (4.4) and using that the sets $I_{n}^{\vee}$ are increasing in view of (4.8), there is $N$ such that

$$
y \in I_{N}^{\vee}(x) \text { and } z \in I_{N}^{\vee}(y) .
$$

Thus, using (4.3), there are open neighborhoods $U_{y}$ of $y$ and $U_{z}$ of $z$ with

$$
U_{y} \subset J_{N}^{\vee}(x) \text { and } U_{z} \in J_{N}^{\vee}(y) \text {. }
$$


We choose any lens-shaped regions $\hat{L}$ and $L$ with $L \ll \hat{L} \supset K_{N}$ and $x \in \stackrel{\circ}{L}$. Combining the first inclusion in (4.10) with (4.2), it follows that $U_{y} \subset L$. Hence $y \in \stackrel{\circ}{L}$, and combining the second inclusion in (4.10) again with (4.2), we conclude that $U_{z} \subset L$. It follows that $U_{z} \subset J_{N}^{\vee}(x)$ and thus $z \in I_{N}^{\vee}(x)$. Using again (4.4) implies that $z \in I^{\vee}(x)$, concluding the proof.

The result of this lemma allows us to introduce the relation $\ll$ on $M \times M$ by the condition that $x \ll y$ if $x=y$ or if $y \in I^{\vee}(x)$. According to Theorem 4.5, this relation is transitive. Forming equivalence classes of points $x, y$ for which $x \ll y$ and $y \ll x$, we obtain the structure of a partially ordered set. Such a structure was already obtained in the setting of causal fermion systems in [11, Sections 5.1 and 5.2] with a different construction. The method here has the advantage that it is conceptually more convincing and works in greater generality. We note that if spacetime is discrete and the sets $\{z \in M \mid x \ll z \ll y\}$ are finite for all $x, y \in M$, one recovers the structure of a causal set (see for example [5]).

We close with two short remarks. We first note that defining the relation $\ll$ on $M \times$ $M$ alternatively by the condition $y \in I^{\vee}(x)$ also gives rise to transitive causal relations. But with this alternative definition, the relation $x \ll x$ need not hold, so that the above equivalence classes might be empty. We also point out that, in general, the closed light cones $J^{\vee}(x)$ do not seem to give rise to a transitive relation.

4.2. Definition of Global Retarded Weak Solutions. We now introduce the notion of global retarded weak solutions of the linearized field equations. A global weak solution $\mathfrak{v} \in L_{\text {loc }}^{2}(M, d \rho)$ is defined by the inhomogeneous weak equation

$$
\langle\Delta \mathfrak{u}, \mathfrak{v}\rangle_{L^{2}(M, d \rho)}=\langle\mathfrak{u}, \mathfrak{w}\rangle_{L^{2}(M, d \rho)} \quad \text { for all } \mathfrak{u} \in \mathfrak{J}_{0}^{\text {vary }} .
$$

For technical simplicity, for the moment we restrict attention to inhomogeneities with compact support, i.e.

$$
\mathfrak{w} \in L_{0}^{2}(M, d \rho),
$$

where $L_{0}^{2}(M, d \rho)$ denotes the square integrable jets with essentially compact support (more general inhomogeneities will be considered in Section 5.1).

It remains to make precise what we mean by a retarded solution. In order to implement the notion that the solution should vanish "in the distant past" we again assume that space-time is compactly hyperbolic. Then we can choose an exhaustion of $M$ by lens-shaped regions $\left(L_{n}\right)_{n \in \mathbb{N}}$, i.e.

$$
L_{1} \subset U_{1} \subset L_{2} \subset U_{2} \subset \cdots \quad \text { and } \bigcup_{n \in \mathbb{N}} U_{n}=M .
$$

We take it as a definition that $\mathfrak{v}$ should be a local $L^{2}$-limit of retarded solutions in the lens-shaped regions $L_{n}$ :

Definition 4.6. Assume that space-time is compactly hyperbolic. A global weak solution $\mathfrak{v} \in L_{\text {loc }}^{2}(M, d \rho)$ of (4.11) with compactly supported inhomogeneity (4.12) is said to be retarded if there is an exhaustion by lens-shaped regions $\left(L_{n}\right)_{n \in \mathbb{N}}$ (4.13) such that the corresponding weak solutions $\mathfrak{v}_{n} \in L^{2}\left(L_{n}, d \rho\right)$ of the Cauchy problem with zero initial data, i.e.

$$
\left\langle\Delta \mathfrak{u}, \mathfrak{v}_{n}\right\rangle_{L^{2}\left(L_{n}, d \rho\right)}=\langle\mathfrak{u}, \mathfrak{w}\rangle_{L^{2}\left(L_{n}, d \rho\right)} \quad \text { for all } \mathfrak{u} \in{\overline{\mathfrak{J}} U_{n}}^{t_{\max }},
$$

converge in $L_{\text {loc }}^{2}(M, d \rho)$ to $\mathfrak{v}$. 
We remark for clarity that, since this definition makes a statement on convergence as $n \rightarrow \infty$, it suffices to consider the Cauchy problem (4.14) for large $n$. In particular, one may restrict attention to the case that the support of $\mathfrak{w}$ is contained in $L_{n}$.

4.3. Constructing Unique Global Weak Retarded Solutions. We now give a procedure for constructing global retarded weak solutions of the linearized field equations and specify all the necessary assumptions. In order to keep the setting as simple as possible, we shall make the following assumption:

Definition 4.7. The Lagrangian has finite range if for every lens-shaped region $L$ inside $U$, the set $U$ can be chosen to be relatively compact.

This assumption could be replaced by suitable decay assumptions on the Lagrangian; for the sake of technical simplicity, we shall not enter such generalizations here.

Let $\mathfrak{w} \in L_{0}^{2}(M, d \rho)$ be a compactly supported jet. Assuming again that $M$ is compactly hyperbolic and using that $M$ is $\sigma$-compact, we can exhaust space-time by a sequence of lens-shaped regions $\left(L_{n}\right)_{n \in N}$ inside space-time regions $\left(U_{n}\right)_{n \in \mathbb{N}}$ with local foliations $\left(\eta_{n, t}\right)_{t \in\left[t_{0}, t_{\max }\right]}$. Moreover, we choose $L_{1}$ such that it contains the support of $\mathfrak{w}$, i.e.

$$
\operatorname{supp} \mathfrak{w} \subset L_{1} \subset U_{1} \subset L_{2} \subset U_{2} \subset \cdots \quad \text { and } \bigcup_{n \in \mathbb{N}} U_{n}=M .
$$

Using property (ii) in Definition 3.1, these lens-shaped regions are indeed nested in the sense of Definition 3.19, Applying the existence result of Theorem 3.15 in each lensshaped region, we obtain a sequence of solutions with zero initial data. Working for convenience with the weak equation (3.39), we obtain a sequence of weak solutions $\mathfrak{v}_{n}$ of (4.14). Our goal is to show that this sequence of weak solutions converges in a suitable sense to the desired solution $\mathfrak{v}$. The difficulty is that the solutions $\mathfrak{v}_{n}$ are not unique (see Proposition 3.16), implying that the solutions do not need to coincide locally. Thus, using again the notion introduced in Section 3.10, we need to control the shielding.

Definition 4.8. Given two lens-shaped regions $L$ and $\hat{L}$, we define the shielding constant by

$$
s(V, L, \hat{L})=\sup \left\{\frac{\|\mathfrak{u}\|_{L^{2}(V, d \rho)}}{\|\mathfrak{u}\|_{L^{2}(L, d \rho)}} \mid \mathfrak{u} \in\left(\chi_{L} \Delta\left(\overline{\mathfrak{J}} U^{t_{\max }}\right)\right)^{\perp} \cap \overline{\operatorname{span}(\mathfrak{K}(\hat{L}), \mathfrak{K}(L))}\right\} .
$$

If the shielding constant vanishes, we obtain shielding in the sense of Definition 3.21, Therefore, the shielding constant quantifies to which extent shielding is violated.

In the following theorem we control the shielding by a condition which involves both the shielding constant and the constant $\Gamma$ in the energy estimate of Proposition 3.5. In order to get finer control of the dependence on the considered space-time region, we introduce the constant $\Gamma(L, \hat{L})$ by modifying the inequality (3.33) to

$$
\|\mathfrak{u}\|_{L^{2}(L)} \leq \Gamma(L, \hat{L})\|\Delta \mathfrak{u}\|_{L^{2}(L)} \quad \text { for all } \mathfrak{u} \in{\overline{\mathfrak{J}_{\hat{U}}}}^{t_{\max }}
$$

(thus this is an estimate in $L$, but the jet space must vanish only in the future of the bigger lens-shaped region $\hat{L})$. Typically, the constant $\Gamma(L, \hat{L})$ stays finite in the limiting case that $L$ is fixed and $\hat{L}$ exhausts the whole space-time. 
Theorem 4.9. Assume that the Lagrangian has finite range and that space-time is compactly hyperbolic. Moreover, assume that the shielding constant goes to zero so fast that every $x \in M$ has an open neighborhood $V$ such that

$$
\sum_{n=1}^{\infty} s\left(V, L_{n}, L_{n+1}\right)\left(\Gamma\left(L_{n}, L_{n}\right)+\Gamma\left(L_{n}, L_{n+1}\right)\right)<\infty .
$$

Then for any compactly supported $\mathfrak{w} \in L^{2}(M, d \rho)$ there is a global retarded weak solution.

Proof. We introduce the subspaces

$$
A:=\Delta\left({\overline{\mathfrak{J} U_{n}}}^{t}{ }^{\max }\right), B:=\operatorname{span}\left(\mathfrak{K}\left(L_{n}\right), \mathfrak{K}\left(L_{n+1}\right)\right) \quad \subset L^{2}\left(L_{n+1}, d \rho\right) .
$$

The consideration in the proof of Proposition 3.22 shows that

$$
\mathfrak{v}_{n+1}-\mathfrak{v}_{n} \in A^{\perp} \cap \bar{B} .
$$

Given $x \in M$, we choose an open neighborhood $V$ such that (4.15) holds. Then, by definition of the shielding constant,

$$
\begin{aligned}
\left\|\mathfrak{v}_{n+1}-\mathfrak{v}_{n}\right\|_{L^{2}(V, d \rho)} & \leq s\left(V, L_{n}, L_{n+1}\right)\left\|\mathfrak{v}_{n+1}-\mathfrak{v}_{n}\right\|_{L^{2}\left(L_{n}, d \rho\right)} \\
& \leq s\left(V, L_{n}, L_{n+1}\right)\left(\Gamma\left(L_{n}, L_{n}\right)+\Gamma\left(L_{n}, L_{n+1}\right)\right)\|\mathfrak{w}\|_{L^{2}(V, d \rho)} .
\end{aligned}
$$

The assumption (4.15) ensures that the sequence $\mathfrak{v}_{n}$ converges in $L^{2}(V, d \rho)$ to a function $\mathfrak{v} \in L^{2}(V, d \rho)$. Since $V$ can be chosen as a small neighborhood of any point $x \in M$, we conclude that $\mathfrak{v}_{n}$ converges in $L_{\text {loc }}^{2}(M, d \rho)$ to $\mathfrak{v} \in L_{\text {loc }}^{2}(M, d \rho)$.

In particular, $\mathfrak{v}_{n}$ converges in $L^{2}$ in the lens-shaped region $L_{2}$ and therefore in $U_{1}$. Using that the Lagrangian has finite range, we can apply Lebesgue's dominated convergence theorem to infer that $\Delta \mathfrak{v}_{n}$ converges in $L^{2}$ in the lens-shaped region $L_{1}$. Hence $\left.\left.\Delta \mathfrak{v}_{n}\right|_{V} \rightarrow \Delta \mathfrak{v}\right|_{V}$ in $L^{2}(V, d \rho)$. Again using that $V$ can be chosen as a small neighborhood of any point $x \in M$, we conclude that $\Delta \mathfrak{v}_{n}$ converges in $L_{\text {loc }}^{2}(M, d \rho)$ to $\Delta \mathfrak{v} \in L_{\text {loc }}^{2}(M, d \rho)$. To summarize,

$$
\mathfrak{v}_{n} \rightarrow \mathfrak{v} \text { in } L_{\text {loc }}^{2}(M, d \rho) \quad \text { and } \quad \Delta \mathfrak{v}_{n} \rightarrow \Delta \mathfrak{v} \text { in } L_{\text {loc }}^{2}(M, d \rho) .
$$

Let us verify that $\mathfrak{v}$ satisfies the weak equation (4.11). Thus let $\mathfrak{u} \in \mathfrak{J}_{0}^{\text {vary }}$. Then there is $n$ such that supp $\mathfrak{u} \subset U_{n}$. Using again that the Lagrangian has finite range, it follows that $\mathfrak{u} \in{\overline{\mathfrak{J}} U_{n+2}}_{t}$ max . Hence (4.11) holds for all $\mathfrak{v}_{\ell}$ and all sufficiently large $\ell$. Using (4.17), we can take the limit $\ell \rightarrow \infty$ to conclude that $\mathfrak{v}$ satisfies (4.11).

Let us briefly discuss condition (4.15). As explained after (3.51), shielding takes place on a microscopic length scale $\delta$. This means that, similar to (3.51), the shielding constant $s\left(V, L_{n}, L_{n+1}\right)$ should decay exponentially on the scale $\delta$ if $n$ is increased. The constant $\Gamma$ of the energy estimate, however, increases exponentially on a macroscopic scale. With this in mind, the bound (4.15) seems unproblematic and easy to verify in the applications.

We finally explain in which sense global retarded weak solutions are unique. We first recall that the equations (4.14) determine the $\mathfrak{v}_{n}$ only up to vectors which are orthogonal to the subspace

$$
\chi_{L}{\overline{\mathfrak{J}} U_{n}}^{t_{\max }} \subset L^{2}(M, d \rho) .
$$

Similar as explained in Section 3.8, this means that the global retarded weak solutions are unique up to microscopic details which we deliberately filtered out by our choice 


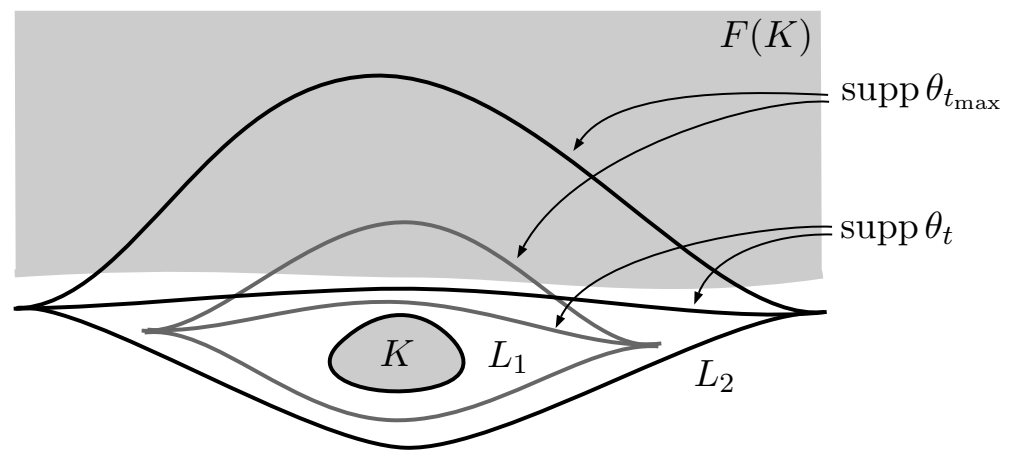

Figure 4. The Cauchy separated future.

of $\mathfrak{J}^{\text {test }}$. Nevertheless, we can hope that, similar as in Theorem 3.15, our construction gives a distinguished solution, which is determined uniquely by our construction. This is indeed the case under suitable assumptions, as we now explain.

Definition 4.10. Space-time has the uniform shielding property if every $x \in M$ has an open neighborhood $V$ such that for any exhaustion by lens-shaped regions $\left(L_{n}\right)_{n \in \mathbb{N}}$ of the form (4.13), the shielding condition (4.15) holds.

Theorem 4.11. If space-time has the uniform shielding property, then the global retarded weak solution of Theorem 4.9 does not depend on the choice of the exhaustion.

Proof. We consider two exhaustions by lens-shaped regions $\left(L_{n}\right)_{n \in \mathbb{N}}$ and $\left(\tilde{L}_{n}\right)_{n \in \mathbb{N}}$. We iteratively choose subsequences $\left(L_{n_{k}}\right)_{k \in \mathbb{N}}$ and $\left(\tilde{L}_{\tilde{n}_{k}}\right)_{k \in \mathbb{N}}$ such that

$$
L_{n_{1}} \subset U_{n_{1}} \subset \tilde{L}_{\tilde{n}_{1}} \subset \tilde{U}_{\tilde{n}_{1}} \subset L_{n_{2}} \subset \cdots \text {. }
$$

We denote the resulting exhaustion by $\left(\hat{L}_{n}\right)_{n \in \mathbb{N}}$. Theorem 4.9 gives a corresponding global retarded weak solution $\hat{\mathfrak{v}}$. This solution coincides with both $\mathfrak{v}$ and $\hat{\mathfrak{v}}$, concluding the proof.

\subsection{Finite Propagation Speed.}

Definition 4.12. Let $K \subset M$ be compact. We let $F(K)$ be the set of space-time points $x$ with the property that there is an open neighborhood $U \subset M$ of $x$ and an exhaustion of $M$ by lens-shaped regions $\left(L_{n}\right)_{n \in N}$ such that for every $n \in \mathbb{N}$ there is $t \in\left[t_{0}, t_{\mathrm{max}}\right]$ with

$$
\left.\eta_{t}\right|_{K} \equiv 1 \quad \text { and }\left.\quad \eta_{t}\right|_{U} \equiv 0 .
$$

We refer to $F(K)$ as the Cauchy separated future of $K$.

Intuitively speaking, the Cauchy separated future of $K$ consists of all points which can be separated from $K$ by surface layers in exhaustions by lens-shaped regions; see Figure 4 ,

Theorem 4.13. Let $\mathfrak{w} \in L_{0}^{2}(M, d \rho)$ with supp $\mathfrak{w} \subset F(K)$. Then there is a global retarded weak solution $\mathfrak{v}$ with $\left.\mathfrak{v}\right|_{K} \equiv 0$.

Proof. Let $x \in F(K)$. We choose an open neighborhood $U$ and an exhaustion $\left(L_{n}\right)_{n \in \mathbb{N}}$ according to Definition 4.12. For any $n$, we choose $t$ such that (4.18) holds. Then the subregion $\tilde{L}:=\cup_{\tilde{t} \in\left[t, t_{\max }\right]} \operatorname{supp} \theta_{\tilde{t}}$ is again a lens-shaped region inside $U_{n}$ with local 
foliation $\left(\eta_{\tilde{t}}\right)_{\tilde{t} \in\left[t, t_{\max }\right]}$. We let $\mathfrak{v}^{+}$be the corresponding solution of Theorem 3.18 with zero initial data at time $t$ and with inhomogeneity $\chi_{U} \mathfrak{w}$. Extending this solution by zero to the past gives a solution in $L_{n}$ which vanishes identically on $K$.

We now consider the resulting sequence $\left(\mathfrak{v}_{n}\right)_{n \in \mathbb{N}}$ of solutions. Using the uniform shielding property (see Definition 4.10), we conclude that this sequence converges in $L_{\text {loc }}^{2}$. We thus obtain a global retarded weak solution $\mathfrak{v}$ with inhomogeneity $\chi_{U} \mathfrak{w}$ which vanishes identically on $K$.

In order to obtain a corresponding solution with inhomogeneity $\mathfrak{w}$, we use linearity and a covering argument: We cover supp $\mathfrak{w}$ by a finite number of open sets $U_{1}, \ldots, U_{L}$ as above and construct corresponding global retarded weak solutions, choosing the inhomogeneity in the $\ell^{\text {th }}$ step as

$$
\mathfrak{w}_{\ell}:=\chi_{U_{\ell} \backslash\left(U_{1} \cup \cdots \cup U_{\ell-1}\right)} \mathfrak{w} .
$$

Adding these solutions gives the desired global retarded weak solution with inhomogeneity $\mathfrak{w}$ which vanishes identically on $K$.

Definition 4.14. Let $L$ be a lens-shaped region inside $U$ with local foliation $\left(\eta_{t}\right)_{t \in\left[t_{0}, t_{\max }\right]}$. The jet space $\mathfrak{J}_{U}$ is future-partitioned by the linear operator $\check{\pi}: \mathfrak{J}_{U} \rightarrow \mathfrak{J}_{U}$ if the following conditions hold:

$$
\begin{aligned}
& \eta_{t_{0}}(1-\check{\pi}) \equiv 0 \equiv\left(1-\eta_{t_{\max }}\right) \check{\pi} \\
& \check{\pi} \mathfrak{u} \in \overline{\mathfrak{J}}_{U}^{t_{\max }} \quad \text { for all } \mathfrak{u} \in \mathfrak{J}_{U} .
\end{aligned}
$$

These conditions mean in words that the zero boundary conditions at time $t_{\max }$ can be realized by acting on jets $\mathfrak{u} \in \mathfrak{J}_{U}$ by the operator $\check{\pi}$.

The support of $\check{\pi}$ (and similarly $(\mathbb{1}-\check{\pi})$ ) is defined by

$$
\operatorname{supp} \check{\pi}:=\overline{\left\{x \in M \mid \exists \mathfrak{u} \in \mathfrak{J}_{U} \text { with }(\check{\pi} \mathfrak{u})(x) \neq 0\right\}} .
$$

Definition 4.15. Space-time is future localizable if for every compact $K \subset M$ there is an exhaustion of $M$ by lens-shaped regions $\left(L_{n}\right)_{n \in N}$ such that for all $n \in \mathbb{N}$ the following condition holds: The jet space $\mathfrak{J}_{U_{n}}$ is future-partitioned by an operator $\check{\pi}_{n}$ such that for all $x \in \operatorname{supp}\left(\mathbb{1}-\check{\pi}_{n}\right)$ and for all $y \in U_{n} \backslash F(K)$, the function $\mathcal{L}(x, y)$ as well as its first and second derivatives in the direction of $\mathfrak{J}_{0}^{\text {vary }}$ vanish.

Intuitively speaking, space-time is future localizable if there is an exhaustion by lensshaped regions such that the future boundaries of the lens-shaped region lie inside and are $\mathcal{L}$-localized in the separated future of $K$ (as shown in Figure 5, see also the notion introduced on page 13).

Theorem 4.16. Assume that space-time is future localizable. Let $\mathfrak{w} \in L_{0}^{2}(M, d \rho)$ be an inhomogeneity with compact support $K:=\operatorname{supp} \mathfrak{w}$. Moreover, let $y \notin J^{\vee}(K)$. Then there is an open neighborhood $W$ of $y$ as well as a global retarded weak solution $\mathfrak{v} \in$ $L_{\text {loc }}^{2}(M, d \rho)$ with

$$
\left.\mathfrak{v}\right|_{W} \equiv 0
$$

Proof. Let $y \notin J^{\vee}(K)$ and $K_{y}$ a compact set whose interior contains $y$. Let $\left(L_{n}\right)_{n \in \mathbb{N}}$ be an exhaustion of $M$ by lens-shaped regions chosen according to Definition 4.15 for the set $K_{y}$. Choose $x \in K$. Then from (4.5) we know that $y \notin J^{\vee}(x)$. Hence, inverting (4.6), we obtain

$$
\underset{N \in \mathbb{N}}{\forall} \underset{\hat{L} \supset K_{N}}{\exists} \underset{L \ll \hat{L}}{\exists} \text { with } \quad x \in \stackrel{\circ}{=} \quad \text { but } \quad y \notin L .
$$




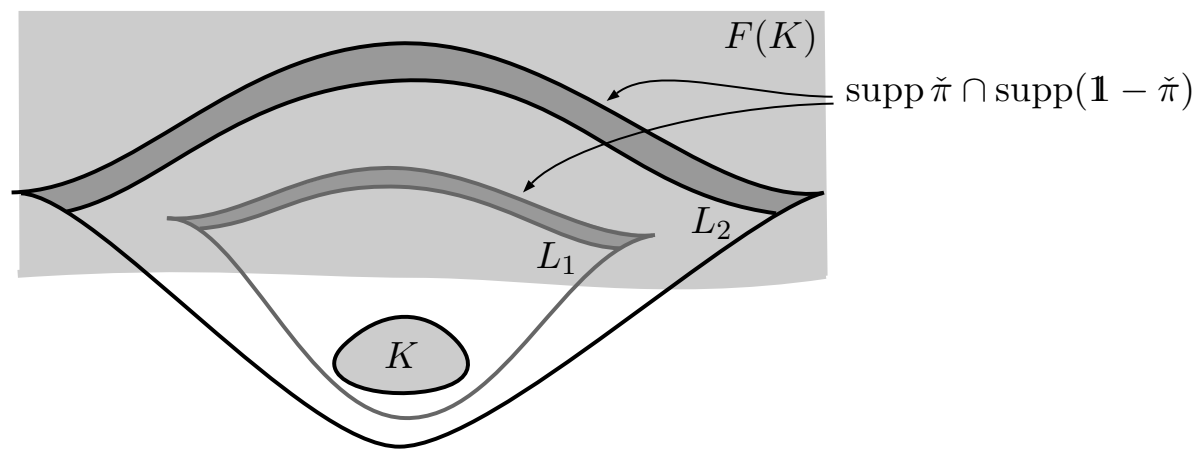

Figure 5. A future-localizing exhaustion.

Since $L$ is closed, we can choose open neighborhoods $U_{x}$ of $x$ and $V_{y} \subset K_{y}$ of $y$ with

$$
\overline{U_{x}} \subset \stackrel{\circ}{L} \quad \text { and } \quad \overline{V_{y}} \subset M \backslash L .
$$

Then the function

$$
\hat{\mathfrak{w}}:=\frac{1}{\eta_{\left[t_{0}, t_{\max }\right]}} \chi_{U_{x}} \mathfrak{w}
$$

is bounded (because $\chi_{U_{x}}$ vanishes near the boundary of $L$ where the function $\eta_{\left[t_{0}, t_{\max }\right]}$ is zero). We let $\hat{\mathfrak{v}}$ be the solution of Theorem 3.15 corresponding to the inhomogeneity $\hat{\mathfrak{w}}$. Then the function

$$
\mathfrak{v}:=\eta_{\left[t_{0}, t_{\max }\right]} \hat{\mathfrak{v}}
$$

is a weak solution in $L^{2}(L, d \rho)$ with zero initial data, i.e.

$$
\langle\Delta \mathfrak{u}, \mathfrak{v}\rangle_{L^{2}(L, d \rho)}=\left\langle\mathfrak{u}, \chi_{U_{x}} \mathfrak{w}\right\rangle_{L^{2}(L, d \rho)} \quad \text { for all } \mathfrak{u} \in \overline{\mathfrak{J}} U^{t_{\max }}
$$

Now let $\mathfrak{u} \in \mathfrak{J}_{0}^{\text {vary }}$. By definition, its restriction to $U$ is in $\mathfrak{J}_{U}$ (but it does not necessarily vanish at time $\left.t_{\max }\right)$. Extending the function $\mathfrak{v}$ by zero to $U$ and choosing the operator $\check{\pi}$ as in Definition 4.14 (again for the set $K_{y}$ ), the support of $\mathbb{1}-\check{\pi}$ does not intersect $U_{x}$. Hence, using that $\mathfrak{v}$ is a weak solution, we obtain

$$
\begin{aligned}
\langle\Delta \mathfrak{u}, \mathfrak{v}\rangle_{L^{2}(U, d \rho)} & =\langle\Delta(\check{\pi} \mathfrak{u}), \mathfrak{v}\rangle_{L^{2}(U, d \rho)}+\langle\Delta((\mathbb{1}-\check{\pi}) \mathfrak{u}), \mathfrak{v}\rangle_{L^{2}(U, d \rho)} \\
& =\left\langle\mathfrak{u}, \chi_{U_{x}} \mathfrak{w}\right\rangle_{L^{2}(U, d \rho)}+\langle\Delta((\mathbb{1}-\check{\pi}) \mathfrak{u}), \mathfrak{v}\rangle_{L^{2}(U, d \rho)},
\end{aligned}
$$

where in the last step we used (4.19) and (4.20). Since both $\mathfrak{v}$ and $\chi_{U_{x}} \mathfrak{w}$ vanish outside $L$, in the last scalar product we may just as well integrate over $M$, if all functions are extended by zero from $U$ to $M$. Moreover, the last summand in (4.21) is linear in the jet $\mathfrak{u}$ and involves this jet only on the support of $(\mathbb{1}-\check{\pi})$. Hence, using that space-time is future localizable (see Definition 4.15), this term can be written as

$$
\langle\Delta((\mathbb{1}-\check{\pi}) \mathfrak{u}), \mathfrak{v}\rangle_{L^{2}(M, d \rho)}=-\left\langle\mathfrak{u}, \mathfrak{w}^{\text {err }}\right\rangle_{L^{2}(M, d \rho)}
$$

with an "error jet" $\mathfrak{w}^{\text {err }}$ supported inside $F\left(K_{y}\right) \cap U$. Therefore, we can apply Theorem 4.13 to obtain a global retarded weak solution with inhomogeneity $\mathfrak{w}^{\text {err }}$ which vanishes on $K_{y}$. Adding this solution to the jet $\mathfrak{v}$, we obtain a global retarded weak solution which vanishes in $\bar{V}_{y}$.

To summarize the result so far, we have shown that for every $y \notin J^{\vee}(K)$ and for every $x \in K$, there are open neighborhoods $U_{x}$ and $V_{y}$ as well as a global weak retarded 
solution $\mathfrak{v}_{x}$ of the equation

$$
\left\langle\Delta \mathfrak{u}, \mathfrak{v}_{x}\right\rangle_{L^{2}(M, d \rho)}=\left\langle\mathfrak{u}, \chi_{U_{x}} \mathfrak{w}\right\rangle_{L^{2}(M, d \rho)} \quad \text { for all } \mathfrak{u} \in \mathfrak{J}_{0}^{\text {vary }}
$$

which vanishes identically in $\bar{V}_{y}$,

$$
\left.\mathfrak{v}_{x}\right|_{\bar{V}_{y}} \equiv 0
$$

Similar as in the proof of Theorem 4.13, we cover $K$ by a finite number of such neighborhoods $U_{x_{1}}, \ldots, U_{x_{L}}$. Summing these solutions and setting $W=V_{x_{1}} \cap \cdots \cap V_{x_{L}}$ gives the result.

Having specified in which sense $J^{\vee}(K)$ determines the propagation speed, we can also generalize other notions familiar from hyperbolic PDEs (see for example [34, Section 8.3]).

Definition 4.17. The domain of influence $\mathcal{J}(K)$ of a compact subset $K \subset M$ is defined by

$$
\mathcal{J}(K)=J^{\vee}(K) \cup J^{\wedge}(K) .
$$

The domain of determination $\mathcal{D}(A)$ of a subset $A \subset M$ is defined by

$$
\mathcal{D}(A)=M \backslash \bigcup\{\mathcal{J}(K) \mid K \subset M \text { compact and } \mathcal{J}(K) \cap A=\varnothing\} .
$$

$A$ set $A \subset M$ is a domain of dependence for the point $x \in M$ if $x \in \mathcal{D}(A)$.

4.5. Globally Hyperbolic Space-Times. The causal relations introduced in Section 4.1 seem somewhat artificial because the condition $y \in J^{\vee}(x)$ may depend on the structure of the lens-shaped regions in an arbitrarily distant space-time region. While it is sensible that the condition $y \in J^{\vee}(x)$ involves the lens-shaped regions in a sufficiently large region containing $x$ and $y$, this region should nevertheless be compact. Moreover, in (4.9) there is the technical complication that even for points $\tilde{y}$ in a small neighborhood of $y$, it might be necessary to choose the parameter $N$ arbitrarily large. Finally, our definitions do not imply that the future cone is a closed subset of $M$. We now introduce a setting which avoids all these subtleties. This definition will not be used in the remainder of this paper, but it might be a suitable starting point for the future.

Definition 4.18. Space-time is causally simple if the following conditions hold:

(i) For all $x \in M$ and every compact set $K \subset M$ there are compact sets $K_{1}$ and $K_{2}$ with $K_{1} \subset K_{2} \subset M$ such that

$$
J^{\vee}(x) \cap K=\left\{y \in K \mid \underset{K_{1} \subset \hat{L} \subset K_{2}}{\forall} \underset{L \ll \hat{L}}{\forall}: x \in \stackrel{\circ}{\forall} \Longrightarrow y \in L\right\} .
$$

(ii) For any compact subset $K \subset M$, the set $J^{\vee}(K)$ is closed in $M$.

The condition (i) implies that the parameter $N$ in (4.6) can be chosen locally uniformly in $y$. As a consequence, in (4.9) the quantifiers may be interchanged to obtain

$$
I^{\vee}(x)=\overbrace{J^{\vee}(x)}^{\circ} .
$$

We thus recover the familiar setting where the open future light cone is the interior of $J^{\vee}$, which in view of (ii) we can refer to as the closed future light cone. Clearly, the name "causally simple" is inspired by the related notion in Lorentzian geometry (see for example [29, Section 6.3] or [32, Section 3.10]), but we point out that the connection between these notions is not more than a superficial analogy. 
Here is another property which does not seem to be satisfied for general minimizers of causal variational principles, but which seems reasonable to impose because it holds in Lorentzian space-times (see for example [32, Proposition 3.38]):

Definition 4.19. The open future cones $I^{\vee}(x)$ are inner continuous if for every compact $K \subset I^{\vee}(x)$ there is an open neighborhood $U_{x}$ of $x$ such that $K \subset I^{\vee}(\tilde{x})$ for all $\tilde{x} \in U_{x}$.

We finally combine previous notions and assumptions to a proposal of what could be a sensible generalization of the class of globally hyperbolic Lorentzian manifolds to the setting of causal variational principles:

Definition 4.20. Space-time is globally hyperbolic if it has the following properties:

(i) Space-time is compactly hyperbolic (see Definition 4.1) and has the uniform shielding property (see Definition 4.10 and likewise for advanced solutions).

(ii) Space-time is causally simple (see Definition 4.18).

(iii) The open cones are inner continuous (see Definition 4.19 and similarly for past cones).

(iv) Space-time is future localizable (see Definition 4.15) and similarly past localizable.

(v) Space-time has compact diamonds, meaning that for all compact $K, K^{\prime} \subset M$, the set $J^{\vee}(K) \cap J^{\wedge}\left(K^{\prime}\right)$ is compact.

We note that here we do not enter the question whether the above assumptions are all independent. We also remark that condition $(\mathrm{v})$ is a stronger condition than imposing that the sets $J^{\vee}(x) \cap J^{\wedge}\left(x^{\prime}\right)$ are compact for all $x, x^{\prime} \in M$, similar as explained for smooth manifolds in [3, Section 1.1]. We finally point out that the above assumptions do not rule out the possibility that the space-time might be non-chronological in the sense that $x \in I^{\vee}(x)$ for some $x \in M$.

4.6. Global Foliations by Cauchy Surface Layers. The previous constructions were based on energy estimates in compact subregions of space-time (more precisely, lens-shaped regions admitting a local foliation satisfying suitable hyperbolicity conditions). By extending local solutions we succeeded in constructing global solutions. But so far we have avoided working with global foliations covering all of space-time. In a globally hyperbolic Lorentzian space-time, global foliations are known to exist (see [2]). Therefore, it seems an interesting question whether a globally hyperbolic space-time (see Definition 4.20) admits global foliations by surface layers. This question is an open problem which goes beyond the scope of the present paper. But we now give a possible definition of a global foliation and indicate how a global foliation could be used for constructing global solutions of the Cauchy problem. We also mention the points which, from our point of view, would be the main difficulties in carrying out this program. Here is a first suggestion for a definition of a global foliation:

Definition 4.21. A function $\eta \in \mathbb{C}^{\infty}(\mathbb{R} \times M, \mathbb{R})$ with $0 \leq \eta \leq 1$ is called a global foliation by Cauchy surface layers if the following conditions hold:

(i) The function $\theta(t,):.=\partial_{t} \eta(t,$.$) is non-negative.$

(ii) The surface layers cover all of $M$ in the sense that

$$
M=\bigcup_{t \in \mathbb{R}} \operatorname{sipp} \theta(t, .) .
$$


(iii) The following hyperbolicity conditions hold: For every $T>0$ there is a constant $C>0$ such that for all $t \in[-T, T]$,

$$
(\mathfrak{v}, \mathfrak{v})^{t} \geq \frac{1}{C} \int_{M}\left(\|\mathfrak{v}(x)\|_{x}^{2}+\left|\Delta_{2}[\mathfrak{v}, \mathfrak{v}]\right|\right) d \rho_{t}(x) \quad \text { for all } \mathfrak{v} \in \mathfrak{J}_{0}^{\text {vary }}
$$

where we again use the notation (3.2) and (3.6), writing $\eta(t, x)$ as $\eta_{t}(x)$ and similarly $\theta(t, x)$ as $\theta_{t}(x)$.

One difficulty is that, since the surface layers are no longer compact, proving the inequality (4.22) makes it necessary to control the behavior of the jets at spatial infinity. Once this rather subtle issue has been settled, one could follow the strategy in Section 3 to prove existence and uniqueness, but now globally in space-time. With this in mind, it would be desirable to work with global foliations. However, as mentioned above, the existence of global foliations is a challenging open problem.

\section{Causal Green's Operators and their Properties}

Having developed the existence theory for global solutions, we can now construct advanced and retarded Green's operators and analyze their properties. In preparation, we extend the existence result for global solutions of Theorem 4.9 to inhomogeneities whose support is not necessarily compact (Section 5.1). Then the causal Green's operators can be defined in a straightforward way (Section 5.2). We finally explain how the difference of the advanced and retarded Green's operator can be used to describe the homogeneous solution space (Section 5.3).

\subsection{Past and Spatially Compact Inhomogeneities.}

Definition 5.1. A jet $\mathfrak{w} \in L_{\text {loc }}^{2}(M, d \rho)$ is called past and spatially compact if its support lies in the causal future of a compact set $K$, i.e

$$
\operatorname{supp} \mathfrak{w} \subset J^{\vee}(K) \text {. }
$$

Similarly, a jet is future and spatially compact) if $\operatorname{supp} \mathfrak{w} \subset J^{\wedge}(K)$.

Corollary 5.2. Under the assumptions of Theorem [4.9, let $\mathfrak{w}$ be a past and spatially compact jet with the property that every $x \in M$ has an open neighborhood $V$ such that for any exhaustion by lens-shaped regions $\left(L_{n}\right)_{n \in \mathbb{N}}$ of the form (4.13), the following shielding condition holds:

$$
\sum_{n=1}^{\infty} s\left(V, L_{n}, L_{n+1}\right)\left(\Gamma\left(L_{n}, L_{n}\right)+\Gamma\left(L_{n}, L_{n+1}\right)\right)\|\mathfrak{w}\|_{L^{2}\left(L_{n}, d \rho\right)}<\infty .
$$

Then there is a global retarded weak solution with inhomogeneity $\mathfrak{w}$.

Proof. Since $M$ is $\sigma$-compact (see the last paragraph of Section 2.1 on page 6 ), we can write $\mathfrak{w}$ as $\mathfrak{w}=\sum_{p=1}^{\infty} \mathfrak{w}^{(p)}$ with compactly supported $\mathfrak{w}^{(p)}$. According to Theorem 4.9, there are corresponding global advanced weak solutions $\mathfrak{v}^{(p)}$. Our task is to show that the series $\sum_{p=1}^{\infty} \mathfrak{v}^{(p)}$ converges in $L_{\text {loc }}^{1}(M, d \rho)$.

Choosing the lens-shaped region $L_{1}$ such that it contains the compact set $K$ with supp $\mathfrak{w} \subset J^{\vee}(K)$, we can arrange that all the $\mathfrak{w}^{(p)}$ vanish at initial time $t_{\text {min }}$ for all lens-shaped regions $L_{1}, L_{2}, \ldots$ This makes it possible to construct all the solutions $\mathfrak{v}^{(p)}$ with the same series of lens-shaped regions, exactly as in the proof of Theorem 4.9. Noting that the estimate (4.16) involves the $L^{2}$-norm of $\mathfrak{w}^{(p)}$, the inequality (5.1) ensures convergence of the series $\sum_{p=1}^{\infty} \mathfrak{v}^{(p)}$. 
Definition 5.3. The space of all past and spatially compact jets (and similarly future and spatially compact sets) which satisfy the shielding condition (5.1) is denoted by $L_{\mathrm{loc}, \mathrm{psc}}^{2}(M, d \rho)$ (and $\left.L_{\mathrm{loc}, \mathrm{fsc}}^{2}(M, d \rho)\right)$.

Obviously, every jet $\mathfrak{v} \in L_{0}^{2}(M, d \rho)$ with essentially compact support is past and spatially compact as well as future and spatially compact. Clearly, the converse is true if we assume that the diamonds are compact. If we assume in addition that space-time has the uniform shielding property (see Definition 4.10), then the shielding condition (5.1) is satisfied for all compactly supported jets. We thus obtain the following result:

Lemma 5.4. Assume that space-time is globally hyperbolic (see Definition 4.20). Then

$$
L_{0}^{2}(M, d \rho)=L_{\mathrm{loc}, \mathrm{psc}}^{2}(M, d \rho) \cap L_{\mathrm{loc}, \mathrm{fsc}}^{2}(M, d \rho) .
$$

5.2. Causal Green's Operators. We again assume that the Lagrangian has finite range (see Definition 4.7). Then $\Delta$ maps compactly supported jets to compactly supported jets,

$$
\Delta: \mathfrak{J}_{0}^{\text {vary }} \rightarrow L_{0}^{2}(M, d \rho) .
$$

Given $\mathfrak{w} \in L_{\text {loc,psc }}^{2}(M, d \rho)$, in Corollary 5.2 we constructed a corresponding retarded solution $\mathfrak{v} \in L_{\text {loc }}^{2}(M, d \rho)$. We define the retarded Green's operator $S^{\wedge}$ by

$$
S^{\wedge}: L_{\text {loc, }, \mathrm{psc}}^{2}(M, d \rho) \rightarrow L_{\text {loc }}^{2}(M, d \rho), \quad \mathfrak{w} \mapsto-\mathfrak{v} .
$$

The advanced Green's operator $S^{\vee}$ is defined similarly,

$$
S^{\vee}: L_{\mathrm{loc}, \mathrm{fsc}}^{2}(M, d \rho) \rightarrow L_{\mathrm{loc}}^{2}(M, d \rho) .
$$

We point out that our construction of causal Green's operators does not rely on any smoothness assumptions of space-time. For a related construction in Lorentzian space-times of low regularity see [30].

We finally analyze the kernel of the Green's operators. Our starting point is the observation that in the global weak equation (4.11), the inhomogeneity $\mathfrak{w}$ can be changed arbitrarily by wave functions in the orthogonal complement of $\mathfrak{J}_{0}^{\text {vary }}$ (with respect to the scalar product $\left.\langle. \mid .\rangle_{L^{2}(M, d \rho)}\right)$. Indeed, denoting this orthogonal complement by $\left(\mathfrak{J}_{0}^{\text {vary }}\right)^{\perp}$, the right side of (4.11) vanishes identically for any $\mathfrak{w} \in\left(\mathfrak{J}_{0}^{\text {vary }}\right)^{\perp}$. This suggests that also the Green's operators should vanish on such wave functions. This is indeed the case, as is shown in the next lemma.

Lemma 5.5. The Green's operators vanish on $\left(\mathfrak{J}_{0}^{\mathrm{vary}}\right)^{\perp}$ in the sense that

$$
\begin{aligned}
& \left(\mathfrak{J}_{0}^{\text {vary }}\right)^{\perp} \cap L_{\text {loc,psc }}^{2}(M, d \rho) \subset \operatorname{ker} S^{\wedge} \\
& \left(\mathfrak{J}_{0}^{\text {vary }}\right)^{\perp} \cap L_{\text {loc }, \text { fsc }}^{2}(M, d \rho) \subset \operatorname{ker} S^{\vee} .
\end{aligned}
$$

Proof. We only prove (5.3), because the proof of (5.4) is similar. Thus let $\mathfrak{w} \in$ $\left(\mathfrak{J}_{0}^{\text {vary }}\right)^{\perp} \cap L_{\text {loc,psc }}^{2}(M, d \rho)$. In view of the limiting procedures in Corollary 5.2 and Theorem 4.9, it suffices to show that the weak solution constructed in Theorem 3.15 in a lens-shaped region $L$ vanishes for sufficiently large $L$. We choose the lens-shaped region $L$ so large that $\mathfrak{w}$ vanishes in the initial time surface layer as well as in its past. Then for any $\mathfrak{u} \in \overline{\mathfrak{J}}_{U} t_{\max }$,

$$
\langle\mathfrak{w}, \mathfrak{u}\rangle_{L^{2}(L)}=\langle\mathfrak{w}, \mathfrak{u}\rangle_{L^{2}(M, d \rho)}=0
$$


(here we made use of the fact that $\mathfrak{u}$ vanishes in the surface layer at time $t_{\text {max }}$; see $(3.26))$. Therefore, the linear functional $\langle\mathfrak{w}, \mathfrak{u}\rangle_{L^{2}(L)}$ vanishes on a dense subset of the Hilbert space $\mathcal{H}$, implying that it is represented by the zero vector $0=V \in \mathcal{H}$. As a consequence, also the weak solution $\mathfrak{v}:=\Delta V$ vanishes.

5.3. The Causal Fundamental Solution and its Properties. The causal fundamental solution is defined by

$$
G=S^{\wedge}-S^{\vee}: L_{0}^{2}(M, d \rho) \rightarrow L_{\mathrm{loc}}^{2}(M, d \rho) .
$$

It maps to homogeneous weak solutions of the linearized field equations.

In the above definitions of $S^{\wedge}, S^{\vee}$ and $G$, we chose the domain of definition as large as possible. For the applications, however, it is convenient to restrict attention to a smaller domain of "nice" jets. To this end, we define

$$
\begin{aligned}
& \mathfrak{J}_{0}^{* *}:=\left\{\mathfrak{u} \in \mathfrak{J}_{0}^{\text {vary }} \mid S^{\vee} \Delta \mathfrak{u}=S^{\wedge} \Delta \mathfrak{u}=-\mathfrak{u}\right\} \\
& \mathfrak{J}_{0}^{*}:=\left\{\mathfrak{u} \in L_{0}^{2}(M, d \rho) \mid S^{\vee} \mathfrak{u}, S^{\wedge} \mathfrak{u} \in \mathfrak{J}^{\text {vary }}\right\} /\left(\mathfrak{J}_{0}^{\text {vary }}\right)^{\perp} \\
& \mathfrak{J}_{\mathrm{sc}}:=\left\{S^{\wedge} \mathfrak{u}_{1}+S^{\vee} \mathfrak{u}_{2} \mid \mathfrak{u}_{1} \in L_{\text {loc }, \text { psc }}^{2}(M, d \rho) \text { and } S^{\wedge} \mathfrak{u}_{1} \in \mathfrak{J}^{\text {vary }},\right. \\
&\left.\mathfrak{u}_{2} \in L_{\text {loc,fsc }}^{2}(M, d \rho) \text { and } S^{\vee} \mathfrak{u}_{2} \in \mathfrak{J}^{\text {vary }}\right\} \\
& \mathfrak{J}_{\mathrm{sc}}^{*}:=\left\{\mathfrak{u}_{1}+\mathfrak{u}_{2} \mid \mathfrak{u}_{1} \in L_{\text {loc,psc }}^{2}(M, d \rho) \text { and } S^{\wedge} \mathfrak{u}_{1} \in \mathfrak{J}^{\text {vary }},\right. \\
&\left.\mathfrak{u}_{2} \in L_{\text {loc,fsc }}^{2}(M, d \rho) \text { and } S^{\vee} \mathfrak{u}_{2} \in \mathfrak{J}^{\text {vary }}\right\} / \\
&\left(\left(\mathfrak{J}_{0}^{\text {vary }}\right)^{\perp} \cap L_{\text {loc,psc }}^{2}(M, d \rho)\right)+\left(\left(\mathfrak{J}_{0}^{\text {vary }}\right)^{\perp} \cap L_{\text {loc,fsc }}^{2}(M, d \rho)\right),
\end{aligned}
$$

where $\left(\mathfrak{J}_{0}^{\text {vary }}\right)^{\perp}$ is again the orthogonal complement of $\mathfrak{J}_{0}^{\text {vary }}$ with respect to the scalar product $\langle. \mid .\rangle_{L^{2}(M, d \rho)}$. Working modulo such jets reflects the general structure of the global weak equation (4.11), where the inhomogeneity $\mathfrak{w}$ can be changed arbitrarily by jets in $\left(\mathfrak{J}_{0}^{\text {vary }}\right)^{\perp}$. Working modulo such jets, in what follows we do not need to distinguish between weak and strong solutions of the linearized field equations, making it possible to work with the relations $\Delta S^{\vee}=\Delta S^{\wedge}=-\mathbb{1}$. Moreover, in view of Lemma 5.5 the causal Green's operators, and consequently also the causal fundamental solution $G$, are well-defined on the equivalence classes obtained by modding out $\left(\mathfrak{J}_{0}^{\text {vary }}\right)^{\perp}$. In the definition of $\mathfrak{J}_{\mathrm{sc}}^{*}$ we again $\bmod$ out $\left(\mathfrak{J}_{0}^{\text {vary }}\right)^{\perp}$, but this time also respecting the decomposition into the sum of a future and a past spatially compact jet. In order to avoid confusion, we also point out that, identifying the jet space with their duals using the pointwise scalar product (3.10), the space $\mathfrak{J}_{0}^{*}$ does in general not agree with $\mathfrak{J}_{0}^{\text {vary }}$, and $\mathfrak{J}_{\mathrm{sc}}^{*}$ does not coincide with $\mathfrak{J}_{\mathrm{sc}}$. The above definitions identify the correct dual jet spaces, independent of the arbitrarily chosen scalar product (3.10).

It follows immediately from the definitions that $G$ maps $\mathfrak{J}_{0}^{*}$ to $\mathfrak{J}_{\mathrm{sc}}$ and that $\Delta$ can be extended to a well-defined operator from $\mathfrak{J}_{\mathrm{sc}}$ to $\mathfrak{J}_{\mathrm{sc}}^{*}$.

Lemma 5.6. The operator $\Delta$ maps $\mathfrak{J}_{0}^{* *}$ to $\mathfrak{J}_{0}^{*}$.

Proof. Let $\mathfrak{u} \in \mathfrak{J}_{0}^{* *}$. Then the jet $\mathfrak{v}:=\Delta \mathfrak{u}$ has the property

$$
S^{\vee} \mathfrak{v}=S^{\vee} \Delta \mathfrak{u}=-\mathfrak{u} \in \mathfrak{J}^{\text {vary }}
$$

and similarly for $S^{\wedge} \mathfrak{v}$. It follows by definition of $\mathfrak{J}_{0}^{*}$ that $\mathfrak{v} \in \mathfrak{J}_{0}^{*}$. 
In the next theorem we combine the properties of the causal fundamental solution in an exact sequence, similar as obtained for linear hyperbolic PDEs in globally hyperbolic space-times in [27, Proposition 8] and [1, Theorem 4.3].

Theorem 5.7. Assume that space-time is globally hyperbolic (see Definition 4.20) and that the Lagrangian has finite range (see Definition [4.7). Then the following sequence is exact:

$$
0 \rightarrow \mathfrak{J}_{0}^{* *} \stackrel{\Delta}{\longrightarrow} \mathfrak{J}_{0}^{*} \stackrel{G}{\longrightarrow} \mathfrak{J}_{\mathrm{sc}} \stackrel{\Delta}{\longrightarrow} \mathfrak{J}_{\mathrm{sc}}^{*} \rightarrow 0 .
$$

Proof. We proceed in several steps:

(i) $\Delta: \mathfrak{J}_{0}^{* *} \rightarrow \mathfrak{J}_{0}^{*}$ is injective: Let $\mathfrak{v} \in \mathfrak{J}_{0}^{* *}$ with $\Delta \mathfrak{v}=0$. Multiplying by $S^{\vee}$ and using again the definition of $\mathfrak{J}_{0}^{* *}$, we conclude that $\mathfrak{v}=-S^{\vee} \Delta \mathfrak{v}=0$.

(ii) The product $G \circ \Delta: \mathfrak{J}_{0}^{* *} \rightarrow \mathfrak{J}_{\mathrm{sc}}$ vanishes: Let $\mathfrak{v} \in \mathfrak{J}_{0}^{* *}$. Then, by definition of $G$ and $\mathfrak{J}_{0}^{* *}$

$$
G \Delta \mathfrak{v}=S^{\wedge} \Delta \mathfrak{v}-S^{\vee} \Delta \mathfrak{v}=-\mathfrak{v}+\mathfrak{v}=0 .
$$

(iii) If $G \mathfrak{u}=0$ for $\mathfrak{u} \in \mathfrak{J}_{0}^{*}$, then $\mathfrak{u}$ can be represented as $\mathfrak{u}=\Delta \mathfrak{v}$ with $\mathfrak{v} \in \mathfrak{J}_{0}^{* *}$ : By definition of $G$ and $\mathfrak{J}_{0}^{*}$, we know that

$$
\mathfrak{v}:=-S^{\vee} \mathfrak{u}=-S^{\wedge} \mathfrak{u} \in \mathfrak{J}^{\text {vary }} .
$$

Lemma 5.4 yields that $\mathfrak{v} \in \mathfrak{J}_{0}^{\text {vary }}$. Finally, the equation $\Delta \mathfrak{v}=\mathfrak{u}$ follows by definition of the Green's operators.

(iv) The product $\Delta \circ G: \mathfrak{J}_{0}^{*} \rightarrow \mathfrak{J}_{\mathrm{sc}}^{*}$ vanishes: This follows immediately from the definition of the Green's operators.

(v) If $\Delta \mathfrak{v}=0$ for $\mathfrak{u} \in \mathfrak{J}_{\text {sc }}$, then $\mathfrak{v}$ can be represented as $\mathfrak{v}=G \mathfrak{u}$ with $\mathfrak{u} \in \mathfrak{J}_{0}^{*}$ : Representing $\mathfrak{v}$ as in the definition of $\mathfrak{J}_{\mathrm{sc}}$, we obtain by definition of the Green's operators

$$
\Delta \mathfrak{v}=\mathfrak{u}_{1}+\mathfrak{u}_{2} \quad \text { with } \mathfrak{u}_{1} \in L_{\text {loc,psc }}^{2}(M, d \rho) \text { and } \mathfrak{u}_{2} \in L_{\text {loc,fsc }}^{2}(M, d \rho) .
$$

Hence $\mathfrak{u}_{1}=-\mathfrak{u}_{2}=:-2 \pi i \mathfrak{u}$ is compactly supported and $S^{\wedge} \mathfrak{u}, S^{\vee} \mathfrak{u} \in \mathfrak{J}^{\text {vary }}$. In other words, $\mathfrak{u} \in \mathfrak{J}_{0}^{*}$. Moreover, $G \mathfrak{u}=\mathfrak{v}$ by construction.

(vi) The operator $\Delta: \mathfrak{J}_{\mathrm{sc}} \rightarrow \mathfrak{J}_{\mathrm{sc}}^{*}$ is surjective: Let $\mathfrak{u} \in \mathfrak{J}_{\mathrm{sc}}^{*}$. According to the definition of $\mathfrak{J}_{\mathrm{sc}}^{*}$, we can represent $\mathfrak{u}$ as

$$
\mathfrak{u}=\mathfrak{u}_{1}+\mathfrak{u}_{2} \quad \text { with } \mathfrak{u}_{1} \in L_{\text {loc,psc }}^{2}(M, d \rho) \text { and } \mathfrak{u}_{2} \in L_{\text {loc,fsc }}^{2}(M, d \rho) .
$$

Then by definition, the jet $\mathfrak{v}:=-S^{\wedge} \mathfrak{u}_{1}-S^{\vee} \mathfrak{u}_{2}$ is in $\mathfrak{J}_{\text {sc }}$. Moreover, $\Delta \mathfrak{v}=\mathfrak{u}$ by definition of the Green's operators.

This concludes the proof.

The image of the operator $G$ in the exact sequence (5.6) are the linearized weak solutions of spatially compact support denoted by

$$
\mathfrak{J}_{\mathrm{sc}}^{\text {lin }}:=G \mathfrak{J}_{0}^{*} \subset \mathfrak{J}^{\text {test }} .
$$

Remark 5.8. We note for completeness that it seems reasonable to extend the above construction to jets which do not have spatially compact support, similarly to the procedure followed in the study of liner hyperbolic PDEs in globally hyperbolic spacetimes, cf. [1. However, the construction also involves difficulties, as we now outline: A jet $\mathfrak{w} \in L_{\text {loc }}^{2}(M, d \rho)$ is called past compact if for any $x \in M$, the intersection

$$
J^{\wedge}(x) \cap \operatorname{supp} \mathfrak{w} \quad \text { is compact . }
$$


Future compact jets are define analogously. A jet is called timelike compact if it is both future and past compact. Then the goal would be to prove in analogy to (5.6) the exact sequence

$$
0 \rightarrow \mathfrak{J}_{\mathrm{tc}}^{* *} \stackrel{\Delta}{\longrightarrow} \mathfrak{J}_{\mathrm{tc}}^{*} \stackrel{G}{\longrightarrow} \mathfrak{J} \stackrel{\Delta}{\longrightarrow} \mathfrak{J}^{*} \rightarrow 0
$$

where the index "tc" denotes jets $\mathfrak{w}$ which are timelike compact and have the property that there are global advanced and retarded weak solutions with inhomogeneity $\mathfrak{w}$. The main difficulty in establishing (5.8) is that, in order to extend the existence result of Corollary 5.2 to jets which do not have spatially compact support, one would have to get uniform control of our estimates near spatial infinity. More precisely, the shielding condition (5.1) seems problematic if $\mathfrak{w}$ grows rapidly at spatial infinity, making it necessary to work out detailed growth conditions at spatial infinity. Moreover, one would have to make sure that there is an exhaustion by lens-shaped regions with the property that the support of $\mathfrak{w}$ lies in the future of every surface layer at initial time $t_{0}$. Exactly as explained at the end of Section 4.6 in the context of global foliations, these are subtle issues which we leave as open problems for future research.

5.4. Connection to the Symplectic Form. In this section we derive an identity involving the causal fundamental solution and the symplectic form (see Proposition 5.10 below). The analogous formula in classical field theory is commonly used when quantizing the field in the algebraic formulation. As we shall see, extending this formula to causal variational principles involves a few subtleties.

The symplectic form is an antisymmetric bilinear form on the linearized solutions (see (2.21) or the "softened version" in (3.7)). In [20] it is shown that if $\mathfrak{u}$ and $\mathfrak{v}$ are linearized solutions and $\Omega$ is compact, then $\sigma^{\Omega}(\mathfrak{u}, \mathfrak{v})$ vanishes. This gives rise to a conservation law if one considers the limiting case that $\Omega$ exhausts the region between two Cauchy surfaces (for a detailed explanation see [19, Section 2.3] and 20. Section 1]). In the present more general setting we do not want to assume the existence of a Cauchy surface. Therefore, we proceed instead as follows. Let $\mathfrak{u}, \mathfrak{v} \in \mathfrak{J}_{0}^{*}$ be two compactly supported jets. By applying the operator $G$ in (5.5) we obtain two linearized solutions $G \mathfrak{u}, G \mathfrak{v} \in \mathfrak{J}_{\mathrm{sc}}^{\text {lin }}$ (see (5.7)). Similar to the procedure in algebraic quantum field theory, we restrict attention to linearized solutions of this form. We again assume that space-time is globally hyperbolic and that the Lagrangian has finite range (see Definitions 4.20 and4.7). Then we can choose a lens-shaped region $L$ inside a relatively compact open subset $U \subset M$ together with an operator $\check{\pi}: \mathfrak{J}_{U} \rightarrow \overline{\mathfrak{J}} U^{t_{\max }}$ which is identically equal to one in the past of a surface layer which lies to the future of the supports of $\mathfrak{u}$ and $\mathfrak{v}$ (see Figure 6, for technical details see the proof of Proposition 5.10 below). For the symplectic form we want to take into account the surface layer integral involving the solutions $G \mathfrak{u}$ and $G \mathfrak{v}$ in the future. For technical simplicity, it is preferable to work with the "softened" surface layer integral, where for the "softening" we work directly with the operator $\check{\pi}$. In order to derive the resulting form of the symplectic form, it is helpful to first write the symplectic form (2.21) in terms of the linearized field operator $\Delta$ as

$$
\sigma^{\Omega}(\mathfrak{u}, \mathfrak{v})=\left\langle\chi_{\Omega} \mathfrak{u}, \Delta\left(\chi_{M \backslash \Omega} \mathfrak{v}\right)\right\rangle_{L^{2}(M, d \rho)}-\left\langle\chi_{M \backslash \Omega} \mathfrak{u}, \Delta\left(\chi_{\Omega} \mathfrak{v}\right)\right\rangle_{L^{2}(M, d \rho)}
$$




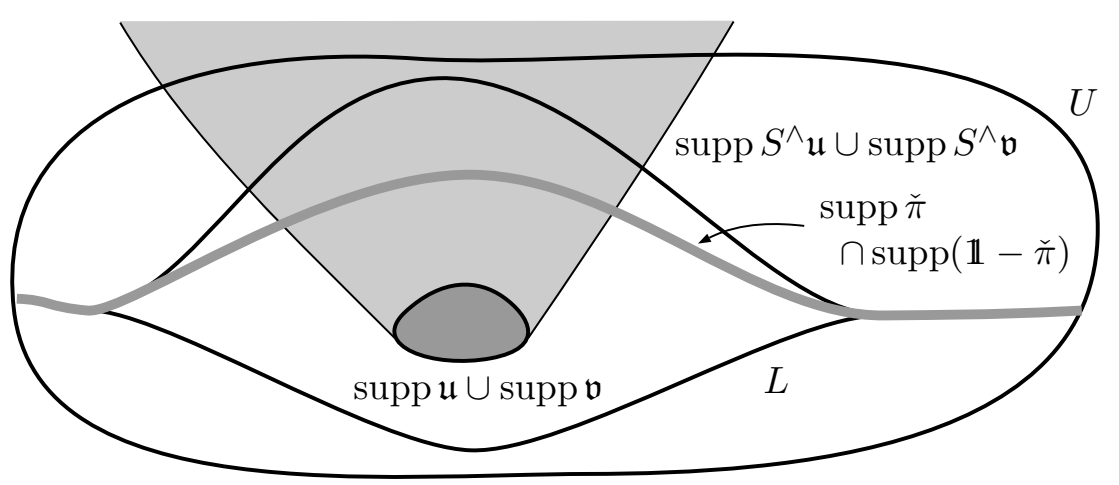

Figure 6. Choice of the lens-shaped region $L$.

(this identity follows from (2.21) and (2.18) by a direct computation). Replacing the characteristic function $\chi_{\Omega}$ leads us to the surface layer integral

$$
\begin{aligned}
& \langle\check{\pi} \mathfrak{u}, \Delta((1-\check{\pi}) \mathfrak{v})\rangle_{L^{2}(M, d \rho)}-\langle(1-\check{\pi}) \mathfrak{u}, \Delta(\check{\pi} \mathfrak{v})\rangle_{L^{2}(M, d \rho)} \\
& =\int_{M} d \rho(x) \int_{M} d \rho(y)\left(\nabla_{1, \check{\pi} \mathfrak{u}} \nabla_{2,(1-\check{\pi}) \mathfrak{v}}-\nabla_{1,(1-\check{\pi}) \mathfrak{u}} \nabla_{2, \check{\pi} \mathfrak{v}}\right) \mathcal{L}(x, y) \\
& \quad+\int_{M}\left(\nabla_{\check{\pi} \mathfrak{u}} \nabla_{(1-\check{\pi}) \mathfrak{v}}-\nabla_{(1-\check{\pi}) \mathfrak{u}} \nabla_{\check{\pi} \mathfrak{v}}\right) \ell(x) d \rho(x),
\end{aligned}
$$

where we used (2.18) and (2.3). For clarity, we point out that if $\check{\pi}$ is replaced by $\chi_{\Omega}$, then (5.11) vanishes, whereas (5.10) gives back the usual formula for the symplectic form (2.21). We want to evaluate this modified symplectic form for the linearized solutions $G \mathfrak{u}$ and $G \mathfrak{v}$ with $\mathfrak{u}, \mathfrak{v} \in \mathfrak{J}_{0}^{*}$. Using (5.5), the contributions by the advanced Green's operators should vanish in the surface layer. In order to avoid the proof of this statement, we simply defining the symplectic form by taking only the retarded contribution,

$$
\sigma^{\check{\pi}}(G \mathfrak{u}, G \mathfrak{v}):=\left\langle\check{\pi} S^{\wedge} \mathfrak{u}, \Delta\left((1-\check{\pi}) S^{\wedge} \mathfrak{v}\right)\right\rangle_{L^{2}(M, d \rho)}-\left\langle(1-\check{\pi}) S^{\wedge} \mathfrak{u}, \Delta\left(\check{\pi} S^{\wedge} \mathfrak{v}\right)\right\rangle_{L^{2}(M, d \rho)}
$$

The remaining task is to simplify this expression and to show that it is independent of the choices of the lens-shaped region $L$ and of the cutoff function $\check{\pi}$. We begin with a preparatory lemma:

Lemma 5.9. For all $\mathfrak{u}, \mathfrak{v} \in \mathfrak{J}_{0}^{*}$,

$$
\left\langle S^{\wedge} \mathfrak{u}, \mathfrak{v}\right\rangle_{L^{2}(M, d \rho)}=\left\langle\mathfrak{u}, S^{\vee} \mathfrak{v}\right\rangle_{L^{2}(M, d \rho)} .
$$

Proof. By definition of the Green's operators,

$$
\left\langle S^{\wedge} \mathfrak{u}, \mathfrak{v}\right\rangle_{L^{2}(M, d \rho)}=-\left\langle S^{\wedge} \mathfrak{u}, \Delta S^{\vee} \mathfrak{v}\right\rangle_{L^{2}(M, d \rho)}
$$

Now we can apply the Green's formula (see Lemma 3.13) in a sufficiently large lensshaped region. Using the support properties of $S^{\vee} \mathfrak{u}$ and $S^{\wedge} \mathfrak{v}$, we do not get boundary terms. Hence

$$
-\left\langle S^{\wedge} \mathfrak{u}, \Delta S^{\vee} \mathfrak{v}\right\rangle_{L^{2}(M, d \rho)}=-\left\langle\Delta S^{\wedge} \mathfrak{u}, S^{\vee} \mathfrak{v}\right\rangle_{L^{2}(M, d \rho)}=\left\langle\mathfrak{u}, S^{\vee} \mathfrak{v}\right\rangle_{L^{2}(M, d \rho)} .
$$

This concludes the proof.

Proposition 5.10. For all $\mathfrak{u}, \mathfrak{v} \in \mathfrak{J}_{0}^{*}$,

$$
\sigma^{\check{\pi}}(G \mathfrak{u}, G \mathfrak{v})=\langle\mathfrak{u}, G \mathfrak{v}\rangle_{L^{2}(M, d \rho)} .
$$


Proof. The first step is to rewrite (5.12) as a volume integral,

$$
\sigma^{\check{\pi}}(G \mathfrak{u}, G \mathfrak{v})=\left\langle\check{\pi} S^{\wedge} \mathfrak{u}, \Delta S^{\wedge} \mathfrak{v}\right\rangle_{L^{2}(M, d \rho)}-\left\langle S^{\wedge} \mathfrak{u}, \Delta\left(\check{\pi} S^{\wedge} \mathfrak{v}\right)\right\rangle_{L^{2}(M, d \rho)} .
$$

Since $\check{\pi}$ maps to $\overline{\mathfrak{J}}_{U}^{t_{\max }}$, we can apply the linearized field equations to obtain

$$
\sigma^{\check{\pi}}(G \mathfrak{u}, G \mathfrak{v})=-\left\langle\check{\pi} S^{\wedge} \mathfrak{u}, \mathfrak{v}\right\rangle_{L^{2}(M, d \rho)}+\left\langle\mathfrak{u}, \check{\pi} S^{\wedge} \mathfrak{v}\right\rangle_{L^{2}(M, d \rho)} .
$$

Using that $\mathfrak{u}$ and $\mathfrak{v}$ vanish on the support of $\mathbb{1}-\check{\pi}$, it follows that

$$
\sigma^{\check{\pi}}\left(S^{\wedge} \mathfrak{u}, S^{\wedge} \mathfrak{v}\right)=-\left\langle S^{\wedge} \mathfrak{u}, \mathfrak{v}\right\rangle_{L^{2}(M, d \rho)}+\left\langle\mathfrak{u}, S^{\wedge} \mathfrak{v}\right\rangle_{L^{2}(M, d \rho)}
$$

Applying Lemma 5.9, we conclude that

$$
\sigma^{\check{\pi}}\left(S^{\wedge} \mathfrak{u}, S^{\wedge} \mathfrak{v}\right)=-\left\langle\mathfrak{u}, S^{\vee} \mathfrak{v}\right\rangle_{L^{2}(L)}+\left\langle\mathfrak{u}, S^{\wedge} \mathfrak{v}\right\rangle_{L^{2}(L)} \stackrel{\text { (5.5) }}{=}\langle\mathfrak{u}, G \mathfrak{v}\rangle_{L^{2}(L)}
$$

Combining this equation with (5.12) gives the result.

From (5.13) one readily sees that the symplectic form does not depend on the choice of the lens-shaped region $L$. If one prefers, one can also take (5.13) as the definition of the symplectic form. Obviously, the symplectic form is anti-symmetric in its two arguments,

$$
\sigma^{\check{\pi}}(G \mathfrak{u}, G \mathfrak{v})=-\sigma^{\check{\pi}}(G \mathfrak{v}, G \mathfrak{u}) .
$$

But we point out that in general it will be degenerate. Therefore, in the present context it would be more appropriate to call $\sigma$ a presymplectic form. It is convenient to also use the standard notation

$$
G(\mathfrak{u}, \mathfrak{v}):=\langle\mathfrak{u}, G \mathfrak{v}\rangle_{L^{2}(M, d \rho)} .
$$

\section{Discussion and Outlook}

We conclude this paper with a few remarks. The general constructions of this paper have the purpose of clarifying the underlying analytic and geometric structures. In order to apply our results in concrete situations, it is a crucial step to verify the hyperbolicity conditions (see Definitions 3.3 or 3.7). Doing so also involves an appropriate choice of the jet space $\mathfrak{J}^{\mathrm{vary}}$ in (3.4). Generally speaking, the smaller $\mathfrak{J}^{\text {vary }}$ is chosen, the easier it is to satisfy the hyperbolicity conditions. The drawback is that the resulting weak solutions are weaker in the sense that fewer jets are allowed for testing. The correct choice of $\mathfrak{J}^{\mathrm{vary}}$ is not merely a technical exercise, but it amounts to identifying those degrees of freedom of the system which have a dynamical behavior in space-time, because only for those degrees of freedom we can hope to satisfy the hyperbolicity conditions. All the other degrees of freedom must be treated with other, non-hyperbolic methods. Since these non-hyperbolic methods do not fit to the topic of this paper, we shall not enter any details but merely illustrate the above considerations by a concrete example.

Example 6.1. (treating the scalar component) Suppose we want to apply our methods to electromagnetic fields for Dirac systems in Minkowski space. In this case, we choose $\rho$ as the Dirac sea vacuum regularized on the scale $\varepsilon$ (for details see [10, Section 1.2] or [33]). In [13] it is shown that the hyperbolicity conditions of Definition 3.3 are satisfied if we choose $\mathfrak{J}^{\text {vary }}$ for example as the jets $\mathfrak{J}^{\text {em }}$ generated by smooth electromagnetic potentials with spatially compact support (for details see [14, Section 7]). However, it is has not yet been analyzed whether the scalar jets also satisfy the hyperbolicity conditions (of either Definition 3.3 or Definition 3.7). Therefore, for the moment the easiest method is to choose $\mathfrak{J}^{\text {vary }}=\mathfrak{J}^{\mathrm{em}}$. Then our energy methods 
apply, giving weak solutions (3.32). However, since the test jets are a subset of $\mathfrak{J}^{\text {vary }}$, we are not allowed to test with scalar jets. In other words, (3.32) does not give us any information on the scalar component of $\Delta \mathfrak{v}$. This is a major shortcoming, because the scalar component of the linearized field equations is essential for the conservation laws for surface layer integrals. Therefore, it is important to extend our methods such as to also satisfy the scalar component of the linearized field equations.

To this end, one can use an iteration method, as we now outline. The above energy methods gives us a jet $\mathfrak{v}=(0, v)$ with no scalar component. We now allow for an additional scalar component $b$ of $\mathfrak{v}$, which we want to choose in such a way that the scalar component of the linearized field equations holds. Indeed, using the weak EL equations (2.9), the scalar component of the linearized field equations can be written as

$$
\int_{M} \mathcal{L}(x, y) b(y) d \rho(y)=\int_{M} D_{2, v} \mathcal{L}(x, y) d \rho(y) .
$$

The integral operator on the left is known to be positive semi-definite (see [25, Lemma 3.5] and [12, Remark 4.2]), and it is strictly positive if restricted to a space of smooth scalar jets which satisfies (2.8). Then we can invert the integral operator in (6.1) to determine $b$.

Clearly, the scalar jet $b$ also has an effect on the vector component of the linearized field equations. However, as is worked out in detail in [17, Appendix B.1], both $b$ and its "back reaction" on the vector component of $\mathfrak{v}$ are extremely small because of scaling factors $\varepsilon m$ (where $m$ denotes the rest mass of the Dirac particles). Therefore, one can apply an iteration method and a fixed-point argument to obtain the desired weak solution of the linearized field equation $\mathfrak{v}$ for test jets $\mathfrak{J}^{\text {test }}$ which also include a scalar component and satisfy (2.8).

We finally discuss the role and significance of causality. Indeed, in this paper we encountered different notions of causality: On the level of the causal variational principle, there was a distinction between timelike and spacelike separation (2.2). When studying the dynamics of linearized waves, on the other hand, we obtained the structure of past and future cones (see Definition 4.3), which gave us a transitive relation "lies in the future of" (see Theorem 4.5) and was compatible with the speed of propagation (see Theorem 4.16). This raises the questions: How is this cone structure related to the causal structure (2.2)? Are these structures compatible or are there differences?

The answers to these questions are rather subtle. Before beginning, we point out that in the so-called continuum limit as worked out in detail in [10, both the causal structure of (2.2) as well as the cone structure of Definition 4.3 agree and go over to the causal structure of Minkowski space. More generally, in [15, Section 5] it was shown that the causal structure (2.2) goes over to the causal structure on a globally hyperbolic space-time if the ultraviolet regularization is removed by taking the limit $\varepsilon \searrow 0$. Therefore, in the limiting case of a classical space-time in which the linearized field equations go over to linear hyperbolic PDEs, all the different notions of causality agree.

Clearly, the main interest in the constructions of the present paper lies in the fact that they also apply to generalized "quantum space-times" in which space-time does not have a manifold structure, and the linearized field equations can not be expressed in terms of PDEs. In this general setting, the precise connection between the causal structure in (2.2) and the cone structures in Definition 4.3 is unclear. We expect that these structures agree "on the macroscopic scale," but at present there is no mathematically precise formulation of this statement. In order to explain the connection in 
some more detail, we note that in the more specific setting of causal fermion systems, in addition to (2.2) there is also a functional $\mathcal{C}: M \times M \rightarrow \mathbb{R}$ which distinguishes a time direction (for details see [10, §1.1.2])

$$
\left\{\begin{array}{cl}
y \text { lies in the future of } x & \text { if } \mathcal{C}(x, y)>0 \\
y \text { lies in the past of } x & \text { if } \mathcal{C}(x, y)<0 .
\end{array}\right.
$$

Combining this functional with (2.2), one could define an alternative cone structure by

$$
I_{\mathcal{L}}^{\vee}(x)=\{y \in M \mid \mathcal{L}(x, y)>0 \text { and } \mathcal{C}(x, y)>0\}
$$

(where the subscript $\mathcal{L}$ indicates that this cone structure is induced directly by the Lagrangian). This definition is easier and more elementary than our previous definition in (4.7). However, it is not clear whether it gives rise to transitive causal relations and whether it is compatible with the propagation speed of linearized solutions. At present, the only result in this direction are the extensive computations in [9] which indicate that if Dirac sea configurations in Minkowski space are regularized and the regularization is adjusted such as to satisfy the EL equations, then the cone structure (6.2) does not seem to give rise to transitive causal relations. But these results seem too special for giving a definitive answer.

The basic difficulty in clarifying the connection between the different cone structures is that our energy estimates are based on hyperbolicity conditions (see Definitions 3.3 or 3.7) which involve positivity properties of certain surface layer integrals. These positivity properties should be related to or be a consequence of the fact that $\rho$ is a minimizer of the causal variational principle. But understanding in detail how this connection comes about and how it is related to the cones in (6.2) remains a challenging open problem.

Acknowledgments: We would like to thank Niky Kamran, Igor Khavkine, Johannes Kleiner, Simone Murro, Marco Oppio and Miguel Sánchez for helpful discussions. We are grateful to Marco Oppio, Johannes Wurm and the referee for useful comments on the manuscript.

\section{REFERENCES}

[1] C. Bär, Green-hyperbolic operators on globally hyperbolic spacetimes, arXiv:1310.0738 [math-ph], Commun. Math. Phys. 333 (2015), no. 3, 1585-1615.

[2] A.N. Bernal and M. Sánchez, On smooth Cauchy hypersurfaces and Geroch's splitting theorem, arXiv:gr-qc/0306108, Commun. Math. Phys. 243 (2003), no. 3, 461-470.

[3] P. Bernard and S. Suhr, Lyapounov functions of closed cone fields: from Conley theory to time functions, arXiv:1512.08410 [math.DG], Commun. Math. Phys. 359 (2018), no. 2, 467-498.

[4] V.I. Bogachev, Measure Theory. Vol. I, Springer-Verlag, Berlin, 2007.

[5] L. Bombelli, J. Lee, D. Meyer, and R.D. Sorkin, Space-time as a causal set, Phys. Rev. Lett. 59 (1987), no. 5, 521-524.

[6] Y. Choquet-Bruhat, General Relativity and the Einstein Equations, Oxford Mathematical Monographs, Oxford University Press, Oxford, 2009.

[7] P.T. Chruściel and J.D.E. Grant, On Lorentzian causality with continuous metrics, arXiv:1111.0400 [gr-qc], Classical Quantum Gravity 29 (2012), no. 14, 145001, 32.

[8] R. Courant and D. Hilbert, Methods of Mathematical Physics. Vol. II: Partial differential equations, (Vol. II by R. Courant.), Interscience Publishers (a division of John Wiley \& Sons), New York-London, 1962.

[9] F. Finster, On the regularized fermionic projector of the vacuum, arXiv:math-ph/0612003, J. Math. Phys. 49 (2008), no. 3, 032304, 60. 
[10] - The Continuum Limit of Causal Fermion Systems, arXiv:1605.04742 [math-ph], Fundamental Theories of Physics, vol. 186, Springer, 2016.

[11] Causal fermion systems: A primer for Lorentzian geometers, arXiv:1709.04781 [mathph], J. Phys.: Conf. Ser. 968 (2018), 012004.

[12] —, Positive functionals induced by minimizers of causal variational principles, arXiv:1708.07817 [math-ph], Vietnam J. Math. 47 (2019), 23-37.

[13] The causal action in Minkowski space and surface layer integrals, arXiv:1711.07058 [math-ph], SIGMA Symmetry Integrability Geom. Methods Appl. 16 (2020), no. 091.

[14] _ Perturbation theory for critical points of causal variational principles, arXiv:1703.05059 [math-ph], Adv. Theor. Math. Phys. 24 (2020), no. 3, 563-619.

[15] F. Finster and A. Grotz, A Lorentzian quantum geometry, arXiv:1107.2026 [math-ph], Adv. Theor. Math. Phys. 16 (2012), no. 4, 1197-1290.

[16] _ On the initial value problem for causal variational principles, arXiv:1303.2964 [math-ph], J. Reine Angew. Math. 725 (2017), 115-141.

[17] F. Finster and N. Kamran, Complex structures on jet spaces and bosonic Fock space dynamics for causal variational principles, arXiv:1808.03177] [math-ph], to appear in Pure Appl. Math. Q. (2020).

[18] F. Finster and J. Kleiner, Causal fermion systems as a candidate for a unified physical theory, arXiv:1502.03587 [math-ph], J. Phys.: Conf. Ser. 626 (2015), 012020.

[19] Noether-like theorems for causal variational principles, arXiv:1506.09076 [math-ph], Calc. Var. Partial Differential Equations 55:35 (2016), no. 2, 41.

[20] _ A Hamiltonian formulation of causal variational principles, arXiv:1612.07192 [math-ph], Calc. Var. Partial Differential Equations 56:73 (2017), no. 3, 33.

[21] _ A class of conserved surface layer integrals for causal variational principles, arXiv:1801.08715 [math-ph], Calc. Var. Partial Differential Equations 58:38 (2019), no. 1, 34.

[22] F. Finster, J. Kleiner, and J.-H. Treude, An Introduction to the Fermionic Projector and Causal Fermion Systems, in preparation, www.causal-fermion-system.com/intro-public.pdf

[23] F. Finster and C. Langer, Causal variational principles in the $\sigma$-locally compact setting: Existence of minimizers, arXiv:2002.04412 [math-ph], to appear in Adv. Calc. Var. (2020).

[24] F. Finster and A. Platzer, A positive mass theorem for static causal fermion systems, arXiv:1912.12995 [math-ph] (2019).

[25] F. Finster and D. Schiefeneder, On the support of minimizers of causal variational principles, arXiv:1012.1589 [math-ph], Arch. Ration. Mech. Anal. 210 (2013), no. 2, 321-364.

[26] K.O. Friedrichs, Symmetric hyperbolic linear differential equations, Comm. Pure Appl. Math. 7 (1954), 345-392.

[27] N. Ginoux, Linear wave equations, Quantum Field Theory on Curved Spacetimes (C. Bär and K. Fredenhagen, eds.), Lecture Notes in Phys., vol. 786, Springer, Berlin, 2009, pp. 95-84.

[28] J.D.E. Grant, M. Kunzinger, C. Sämann, and R. Steinbauer, The future is not always open, arXiv:1901.07996 [math.DG], to appear in Lett. Math. Phys. (2019).

[29] S.W. Hawking and G.F.R. Ellis, The Large Scale Structure of Space-Time, Cambridge University Press, London, 1973.

[30] G. Hoermann, Y. Sanchez Sanchez, C. Spreitzer, and J. Vickers, Green operators in low regularity spacetimes and quantum field theory, arXiv:1910.13789 [gr-qc] (2019).

[31] F. John, Partial Differential Equations, fourth ed., Applied Mathematical Sciences, vol. 1, Springer-Verlag, New York, 1991.

[32] E. Minguzzi and M. Sánchez, The causal hierarchy of spacetimes, Recent developments in pseudoRiemannian geometry, arXiv:gr-qc/0609119, ESI Lect. Math. Phys., Eur. Math. Soc., Zürich, 2008, pp. 299-358.

[33] M. Oppio, On the mathematical foundations of causal fermion systems in Minkowski space, arXiv:1909.09229 [math-ph], to appear in Ann. Henri Poincaré (2020).

[34] A.D. Rendall, Partial Differential Equations in General Relativity, Oxford Graduate Texts in Mathematics, vol. 16, Oxford University Press, Oxford, 2008.

[35] H. Ringström, The Cauchy Problem in General Relativity, ESI Lectures in Mathematics and Physics, European Mathematical Society (EMS), Zürich, 2009.

[36] M.E. Taylor, Partial Differential Equations. III, Applied Mathematical Sciences, vol. 117, Springer-Verlag, New York, 1997. 
Dipartimento di Fisica, Università degli Studi di Pavia, and infN, Sezione di Pavia, Via Bassi, 6 - I-27100 PaVia, Italy

Email address: claudio.dappiaggi@unipv.it

FAkultät für Mathematik, Universität Regensburg, D-93040 Regensburg, Germany

Email address: finster@ur.de 